\title{
Qualitative Determinacy and Decidability of Stochastic Games with Signals
}

\author{
Nathalie Bertrand \\ INRIA, Centre Bretagne Atlantique, Rennes, France \\ Blaise Genest \\ CNRS, IRISA, Rennes, France \\ Hugo Gimbert \\ CNRS, LaBRI, Bordeaux, France
}

\begin{abstract}
We consider two-person zero-sum stochastic games with signals and finitely many states and actions, a standard model of stochastic games with imperfect information. The only source of information for the players are the signals they receive, they cannot directly observe the state of the game, nor the actions played by their opponent, nor their own actions.

We are interested in the existence of almost-surely winning or positively winning strategies, under reachability, safety, Büchi or co-Büchi winning objectives and the computation of these strategies. We prove two qualitative determinacy results. First, in a reachability game either player 1 can achieve almost-surely the reachability objective, or player 2 can achieve surely the dual safety objective, or both players have positively winning strategies. Second, in a Büchi game if player 1 cannot achieve almost-surely the Büchi objective, then player 2 can ensure positively the dual co-Büchi objective. We prove that players only need strategies with finite-memory. The number of memory states needed to win with finite-memory strategies ranges from one (corresponding to memoryless strategies) to doubly-exponential, with matching upper and lower bounds. Together with the qualitative determinacy results, we also provide fix-point algorithms for deciding which player has an almost-surely winning or a positively winning strategy and for computing an associated finite-memory strategy. Complexity ranges from EXPTIME to 2EXPTIME, with matching lower bounds. Our fix-point algorithms also enjoy a better complexity in the cases where one of the players is better informed than their opponent.

Our results hold even when players do not necessarily observe their own actions. The adequate class of strategies in this case is mixed or general strategies (they are equivalent). Behavioural strategies are too restrictive to guarantee determinacy: it may happen that one of the players has a winning general strategy but none of them has a winning behavioural strategy. On the other hand, if a player can observe their actions, then general, mixed and behavioural strategies are equivalent. Finite-memory strategies are sufficient for determinacy to hold, provided that randomised memory updates are allowed.
\end{abstract}

Categories and Subject Descriptors: B.6.3 [Logic Design]: Design Aids; D.2.4 [Software Engineering]: Software/Program Verification-Model checking; G.3 [Probability and Statistics]: Markov Processes; F.3.1 [Logics and Meanings of Programs]: Specifying and Verifying and Reasoning about Programs

General Terms: Automatic synthesis

Additional Key Words and Phrases: Stochastic games, Controller Synthesis, Imperfect information

The authors acknowledge support from ANR projet STOCH-MC (ANR-13-BS02-0011-01), and the ESF Research Networking Programme project "GAMES2". 


\section{INTRODUCTION}

Numerous advances in algorithmics of stochastic games have recently been made de Alfaro et al. 2007; de Alfaro and Henzinger 2000, Chatterjee et al. 2004, Chatterjee et al. 2005; Gimbert and Horn 2008; Horn 2008, motivated in part by application in controller synthesis and verification of open systems. Open systems can be viewed as two-player games between the system and its environment. At each round of the game, both players independently and simultaneously choose actions and the two choices together with the current state of the game determine transition probabilities to the next state of the game. Properties of open systems are modelled as objectives of the games de Alfaro and Henzinger 2000; Grädel et al. 2002 , and strategies in these games represent either controllers of the system or behaviours of the environment.

Most algorithms for stochastic games suffer from the same restriction: they are designed for games where players can fully observe the state of the system (e.g. concurrent games de Alfaro et al. 2007, de Alfaro and Henzinger 2000 and stochastic games with perfect information [Condon 1992; Horn 2008]). The full observation hypothesis can hinder interesting applications in controller synthesis, actually in most controllable open systems full monitoring for the controller is not implementable in practice. For example the controller of an autonomous driverless subway system cannot directly observe an hardware failure and is only informed about failures detected by the monitoring system, including false alarm due to sensors failures. Moreover, giving full information to the environment is not realistic either. Consider the following example inspired from collision regulation in ethernet protocols: the controller has to share the ethernet layer with the environment, both of them are trying to send a data packet. For that the controller selects a date in microseconds between 1 and 512 then the environment does the same, and then both of them try to send their data packet at the date they chose. Choosing the same date results in a data collision, and the process is repeated until there is no collision, at that time the data can be sent. If the environment has full observation, he knows which date has been chosen by the controller and he is able to create collisions on purpose ad infinitum, which prevents the controller to send his data. However if the date chosen by the controller is kept secret, then the environment cannot prevent the data to be sent eventually almost-surely.

In the present paper, we consider stochastic games with signals, that are a standard tool in game theory to model imperfect information in stochastic games Sorin 2002, Rosenberg et al. 2003 Renault 2007. When playing a stochastic game with signals, players cannot observe the actual state of the game, nor the actions played by themselves or their opponent: the only source of information of a player are private signals they receive throughout the play. Stochastic games with signals subsume standard stochastic games Shapley 1953, repeated games with incomplete information Aumann 1995, games with imperfect monitoring Rosenberg et al. 2003, concurrent games de Alfaro and Henzinger 2000 and deterministic games with imperfect information on one side Reif 1979; Chatterjee et al. 2007 .

Intuitively, players make their decisions based upon the sequence of signals they receive, which is formalised with strategies. As explained in Cristau et al. 2010, some care has to be given to the way strategies are formalised as mathematical objects. Players may play using behavioural strategies, which are mappings from sequences of signals to probability distributions over actions Aumann 1964. This includes in particular the case of pure strategies where the actions are chosen deterministically, i.e. the distributions are Dirac over a single signal.

A more general class of strategies are mixed strategies which are probability measures over the set of pure strategies. When each player observes their own actions, Kuhn's theorem states that behavioural strategies have the same strategic power than mixed strategies $\mathrm{Au}-$ mann 1964. However Kuhn's theorem does not apply when actions are non-observable $\mathrm{Au}-$ 
mann 1964 Cristau et al. 2010. Intuitively, in stochastic games with signals, it may be necessary for the players to base their strategies on the outputs of random generators, kept secret from their adversary, which is not always possible to do with behavioural strategies. For this reason, in the present paper, players are playing with general strategies, which are probability measures over the set of randomised behavioural strategies.

We show that general and mixed strategies are equivalent and, essentially, games with general strategies and non-observable actions are strategically and algorithmically equivalent to games with behavioural strategies and observable actions. Precisely, in a game with non-observable actions, a player has a winning general strategy if and only if the player has a winning behavioural strategy when she is allowed to observe her own actions (Theorem 4.9p. This holds not only for Büchi games but for every games with a Borel winning condition. Moreover, if there exist winning finite-memory strategies in the game with observable actions, they can be transformed to winning finite-memory strategies in the game with non-observable actions with very limited impact on the size of the memory.

From the algorithmic point of view, focusing on games with $\omega$-regular winning conditions, stochastic games with signals are considerably harder to deal with than stochastic games with full observation. While values of the latter games are computable de Alfaro and Henzinger 2000, Chatterjee et al. 2005, simple questions like 'is there a strategy for player 1 which guarantees winning with probability more than $\frac{1}{2}$ ?' are undecidable even for the restricted class of stochastic reachability games with a single signal and a single player $\mathrm{Paz}$ 1971. Also, for this restricted class corresponding to Rabin's probabilistic automata Rabin 1963, the value 1 problem is undecidable Gimbert and Oualhadj 2010. In the present paper, rather than quantitative properties (i.e. questions about values), we focus on qualitative properties of stochastic games with signals.

We study the following qualitative questions about stochastic games with signals, equipped with reachability, safety or Büchi objectives:

(i) Does player 1 have an almost-surely winning strategy, i.e. a strategy which guarantees the objective to be achieved with probability 1, whatever the strategy of player 2 ?

(ii) Does player 2 have a positively winning strategy, i.e. a strategy which guarantees the opposite objective to be achieved with strictly positive probability, whatever the strategy of player 1 ?

Obviously, given an objective, properties (i) and (ii) cannot hold simultaneously. We obtain the following results:

(1) Either property (i) holds or property (ii) holds; in other words these games are qualitatively determined.

(2) Players only need strategies with finite-memory. Depending on the class of objective, the number of memory states needed ranges from one (memoryless) to doubly-exponential.

(3) Questions (i) and (ii) are decidable. We provide fix-point algorithms for computing all initial states that satisfy (i) or (ii), together with the corresponding finite-memory strategies. The complexity of the algorithms ranges from EXPTIME to 2EXPTIME.

(4) The general case of games with non-observable actions and general strategies is reducible to the case of games with observable actions and behavioural strategies.

The first three results are detailed in Theorems 6.1, 6.6, 8.2 and 8.3 . We prove that these results are tight and robust in several aspects. Games with co-Büchi objectives are absent from this picture, since they are neither qualitatively determined (see Section 7.2) nor decidable (as shown in Baier et al. 2008, Chatterjee et al. 2010]).

Another surprising fact is that for winning positively a game with safety or co-Büchi objective, a player needs a memory with a doubly-exponential number of states, and the corresponding decision problem is 2EXPTIME-complete. This result contrasts with previous 
results about stochastic games with imperfect information Reif 1979: Chatterjee et al. 2007, where both the number of memory states and the complexity are simply exponential. Our contributions also reveal a nice property of reachability games: every initial state is either almost-surely winning for player 1 , surely winning for player 2 or positively winning for both players.

Our results strengthen and generalise in several ways results that were previously known for concurrent games de Alfaro et al. 2007, de Alfaro and Henzinger 2000 and deterministic games with imperfect information on one side Reif 1979; Chatterjee et al. 2007].

First, the framework of stochastic games with signals strictly encompasses all the settings of Reif 1979, de Alfaro et al. 2007; de Alfaro and Henzinger 2000 Chatterjee et al. 2007. In concurrent games there is no signalling structure at all, and in deterministic games with imperfect information on one side [Chatterjee et al. 2007] transitions are deterministic and player 2 observes everything that happens in the game, including the actions played by his opponent.

We believe that the extension of results of Chatterjee et al. 2007 to games with imperfect information on both sides is necessary to perform controller synthesis on real-life systems. The collision protocol example described above suggests that simple robust protocols may be not be robust against attacks of an omniscient environment, unless their are allowed to hide information from the environment.

Second, we prove that Büchi games are qualitatively determined: when player 1 cannot win almost-surely a Büchi game then her opponent can win positively. This was not known previously, even for games with imperfect information on one side: in Reif 1979, Chatterjee et al. 2007 algorithms are given for deciding whether the imperfectly informed player has an almost-surely winning strategy for a Büchi (or reachability) objective, however, no results (e.g.: strategy for the opponent) are given in case this player has no such strategy. Our qualitative determinacy result (1) is a radical generalisation of the same result for concurrent games de Alfaro and Henzinger 2000, Th.2], using different techniques. Interestingly, for concurrent games, qualitative determinacy holds for every omega-regular objectives de Alfaro and Henzinger 2000, while for games with signals we show that it fails already for co-Büchi objectives. Interestingly also, stochastic games with signals and a reachability objective have a value Renault and Sorin 2008 but this value is not computable Paz 1971, whereas it is computable for concurrent games with omega-regular objectives de Alfaro and Majumdar 2001. The use of randomised strategies is mandatory for achieving determinacy results, this also holds for stochastic games without signals Shapley 1953, de Alfaro et al. 2007 and even matrix games von Neumann and Morgenstern 1944], which contrasts with Berwanger et al. 2008: Reif 1979 where only deterministic strategies are considered.

Qualitative determinacy is a crucial property of stochastic games when used for controller synthesis, because it allows for incremental design and refinement of systems models and controllers. In case a model of a system (say the door control system of the driverless subway in Paris) does not have a correct controller, qualitative determinacy implies that the environment has a strategy to beat the controller. Such an environment strategy can be used to perform simulation and get error traces, and we believe this can be of great help for the system designers. In case where the environment strategy is not implementable on the actual system then the corresponding restrictions on the environment behaviour should be added to the model of the system. Otherwise, the system itself should be modified in order to defeat this particular environment strategy. Without qualitative determinacy, the designer is left with no feedback when the algorithm answers that there is no winning strategy for the system, and this is a serious limitation to the industrial use of automatic controller synthesis.

Qualitative determinacy has also a strong theoretical interest. The study of zero-sum stochastic games is usually focused on the existence of the value of games: the value is the 
threshold payoff which is a minimal income for player 1 and a maximal loss for player 2, when playing with optimal strategies. The existence of a value is a clue that the strategy sets of the players are rich enough to let them play efficiently, for example deterministic strategies which do not use random coin tosses are too restrictive to play a rock-paperscissors game. The synthesis of almost-surely winning strategies is not related to the notion of value since there are games with value 1 but no almost-surely winning strategies. In our opinion, qualitative determinacy is the key notion of determinacy for almost-surely winning strategies and the key criterion to check that the players are given sets of strategies which are not too restricted.

From this perspective, our qualitative determinacy result shows that general strategies (or equivalently mixed strategies) and finite-memory strategies with randomised memory updates are the right class of strategies to play stochastic games with signals. Indeed, if players are restricted to use behavioural strategies or finite-memory strategies with deterministic memory updates then qualitative determinacy does not hold anymore, as demonstrated by the counter-example in Section 2.6

Our results about winning finite-memory strategies (2), stated in Theorem 6.6, are either brand new or generalise previous work. It was shown in Chatterjee et al. 2007 that for deterministic games where player 2 is perfectly informed, strategies with a finite memory of exponential size are sufficient for player 1 to achieve a Büchi objective almost-surely. We extend these results to the case where player 2 has partial observation too. Moreover, we prove that for player 2 a doubly-exponential number of memory states is necessary and sufficient to achieve positively the dual co-Büchi objective.

Concerning algorithmic results (3) (see details in Theorem 8.2 and 8.3 we give a fix-point based algorithm for deciding whether a player has an almost-surely winning strategy for a Büchi objective. If it is the case, a strategy for achieving almost-surely the Büchi objective (with an exponential number of memory states) can be derived easily. If it is not the case, a strategy (with a doubly exponential number of memory states) for player 2 to prevent the Büchi objective with positive probability can be derived easily. Our algorithm with 2EXPTIME complexity is optimal since the problem is indeed 2EXPTIME-hard (see Theorem 10.1). The same algorithm is also optimal, and with an EXPTIME complexity, under the hypothesis that player 2 has more information than player 1 . This generalises the EXPTIME-completeness result of Chatterjee et al. 2007], in the case where player 2 has perfect information. Last our algorithm also runs in EXPTIME when player 1 has full information. In both subcases, player 2 needs only exponential memory (see Proposition 10.2).

A refined version of Büchi objectives has been introduced in Tracol 2011: instead of requiring infinitely many visits to accepting states, it asks that the limit average of visits to accepting states is positive. Considering this winning condition for the restricted class of probabilistic automata (which correspond to single player stochastic games in which the player is blind) makes the positively-winning set of states computable, contrary to probabilistic automata equipped with a standard Büchi condition. However, whether such a condition can be ensured almost-surely is still undecidable.

An algorithm for deciding whether player 1 wins almost-surely a Büchi game with imperfect information has been obtained in Gripon and Serre 2009 Gripon and Serre 2011, concurrently to our own work. We go one step further since we show qualitative determinacy and we compute not only the almost-surely winning strategies of player 1 but also the positively winning strategies of player 2 .

Moreover in the present paper we do not assume a priori that a player observes their own actions. This requires to use the most general class of finite-memory strategies where the memory updates are randomised, by contrast with finite-memory strategies with deterministic updates used in Gripon and Serre 2009. In a nutshell, we prefer general finite-memory strategies because mimicking randomness with deterministic transitions can be very costly, 
or even not possible. First, finite-memory strategies with randomised updates are strictly more expressive than those with deterministic updates: qualitative determinacy does not hold anymore if players are restricted to finite-memory strategies with deterministic updates Cristau et al. 2010]. Second, general finite-memory strategies are more compact: a memory of non-elementary size is needed in general to win stochastic games with finitememory strategies with deterministic updates Chatterjee and Doyen 2012, while in the present paper we obtain doubly-exponential memory upper bounds when using general finite-memory strategies.

The paper is organised as follows. In Section 2 we introduce stochastic games with signals and we define the notion of qualitative determinacy. . In Section 3 we give examples. In Section 4 we show that games with general strategies and non-observable actions are essentially the same as games with behavioural strategies and observable actions. In Section 5 we introduce belief strategies in games with observable actions. The main results (qualitative determinacy, memory complexity and algorithmic complexity) are stated in Section 6 and proved in the next sections, Section 7 for determinacy, Section 8 for algorithmic results and upper bound on the memory and the complexity, Section 9 for the lower bounds on the memory and Section 10 for the lower complexity bounds.

This paper is an extended version of [Bertrand et al. 2009]. In particular, there are three novelties: we present an extended comparison between behavioural and general strategies, including a reduction from games with non-observable actions and general strategies to games with observable actions and behavioural strategies, we provide a direct proof of qualitative determinacy and our results hold in the general case where the players cannot observe their actions. Moreover complete proofs are provided.

\section{STOCHASTIC GAMES WITH SIGNALS.}

We consider the standard model of finite two-person zero-sum stochastic games with signals Sorin 2002, Rosenberg et al. 2003 Renault 2007]. These are stochastic games where players cannot observe the actual state of the game, nor the actions played by themselves and their opponent; their only source of information are private signals they receive throughout the play. However, since the players know the transitions and in particular the signalling structure of the game, their private signals give them some clues about the information hidden from them. Stochastic games with signals subsume standard stochastic games Shapley 1953, repeated games with incomplete information Aumann 1995, games with imperfect monitoring Rosenberg et al. 2003, games with imperfect information Chatterjee et al. 2007; Gripon and Serre 2009 and partial-observation stochastic games [Chatterjee et al. 2013 .

Notations. Given a finite or countable set $K$, we denote by $\Delta(K)=\{\delta: K \rightarrow[0,1] \mid$ $\left.\sum_{k} \delta(k)=1\right\}$ the set of probability distributions on $K$. For every distribution $\delta \in \Delta(K)$, we denote $\operatorname{supp}(\delta)=\{k \in K \mid \delta(k)>0\}$ its support. For every state $k \in K$, we denote $\mathbf{1}_{k}$ the unique distribution whose support is the singleton $\{k\}$. In general, when a set $S$ is equipped with a $\sigma$-algebra, we denote $\Delta(S)$ the set of probability measures on $S$.

States, actions, signals and arenas. Two players called 1 and 2 have opposite goals and play for an infinite sequence of steps, choosing actions and receiving signals. Players observe the signals they receive but they cannot observe the actual state of the game, nor the actions that are played nor the signals received by their opponent. We assume player 1 to be female and player 2 to be male.

An arena is a tuple $(K, I, J, C, D, p)$, where $K$ is the set of states, $I$ and $J$ are the sets of actions of player 1 and player $2, C$ and $D$ are the sets of signals of player 1 and player 2, and

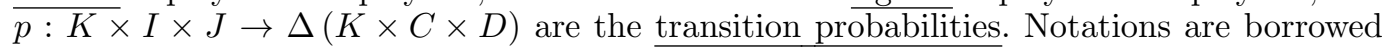
from Renault 2007. 


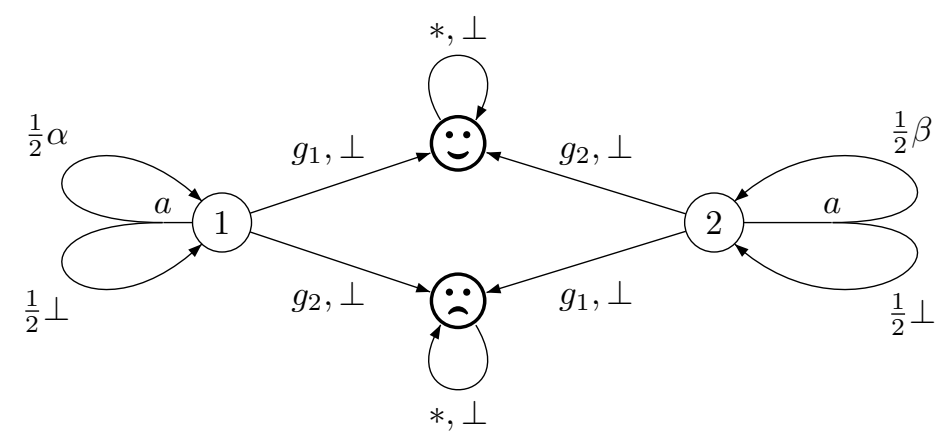

Fig. 1. A one-player stochastic game with signals.

Initially, the game is in a state $k_{0} \in K$ chosen according to an initial distribution $\delta \in$ $\Delta(K)$ known by both players; the initial state is $k_{0}$ with probability $\delta\left(k_{0}\right)$. At each step $n \in \mathbb{N}$, players 1 and 2 choose some actions $i_{n} \in I$ and $j_{n} \in J$. They respectively receive signals $c_{n} \in C$ and $d_{n} \in D$, and the game moves to a new state $k_{n+1}$. This happens with probability $p\left(k_{n+1}, c_{n+1}, d_{n+1} \mid k_{n}, i_{n}, j_{n}\right)$. This fixed probability is known by both players, as well as the whole description of the game.

We provide two examples of stochastic games with signals.

Example 2.1. The first example is a one-player game. It is depicted on Fig. 1.

Actions of player 1 are $I=\left\{a, g_{1}, g_{2}\right\}$, and her signals are $C=\{\alpha, \beta, \perp\}$. Player 2 has a single action and a single signal which are not represented. Transitions probabilities represented on Fig. 1 are interpreted in the following way. When the game is in state 1 and player 1 plays $a$ then player 1 receives signal $\alpha$ or $\perp$, each with probability $\frac{1}{2}$ and the game stays in state 1 . In state 2 when action of player 1 is $a$ then player 1 cannot receive signal $\alpha$ but instead she may receive signal $\beta$. The star symbol $*$ stands for any action: states $(;)$ and $\odot$ are absorbing.

The objective of player 1 is to reach the $\odot-$-state. The initial distribution is $\delta(1)=\delta(2)=\frac{1}{2}$ and $\delta(\odot)=\delta(\odot)=0$.

In order to reach the state $(-)$, player 1 has to correctly "guess the state", i.e. player 1 should play action $g_{1}$ in state 1 and action $g_{2}$ in state 2. Otherwise the game gets stuck in the state $(-)$ from where there is no way to ever reach $(;$.

Example 2.2. The second example is depicted on Fig. 2. The initial state is init. Player 1 has actions $I=\{a, b\}$ and receives two signals $C=\{0,1\}$ while player 2 has actions $J=\left\{a^{\prime}, b^{\prime}\right\}$ and receives only one signal $D=\{\perp\}$. Again, the symbol $*$ stands for "any action". For example, from state $a$, whenever player 1 plays action $a$ then whatever action is chosen by player 2 the next state is $(;)$ and player 1 receives signal $\perp$.

Again, the objective of player 1 is to reach the $\odot$-state. For that she should do two things. First exit the set of states $\{$ init,$\neq\}$. For that player 1 should match the action of player 2 , at an even step, by playing $a$ at the same time player 2 plays $a^{\prime}$ or by playing $b$ at the same time player 2 plays $b^{\prime}$. Then player 1 should play again the same action in order to reach $\odot$.

Plays. A finite play is a sequence $\pi=\left(k_{0}, i_{0}, j_{0}, c_{1}, d_{1}, k_{1}, \ldots, c_{n}, d_{n}, k_{n}\right) \in(K I J C D)^{*} K$ such that for every $0 \leq m<n, p\left(k_{m+1}, c_{m+1}, d_{m+1} \mid k_{m}, i_{m}, j_{m}\right)>0$. An infinite play is a sequence in $(K I J C D)^{\omega}$ such that each prefix in $(K I J C D)^{*} K$ is a finite play.

Strategies. At each step of a game, both players face a choice: they have to select an action. The way players select those actions is represented by a mathematical object called 


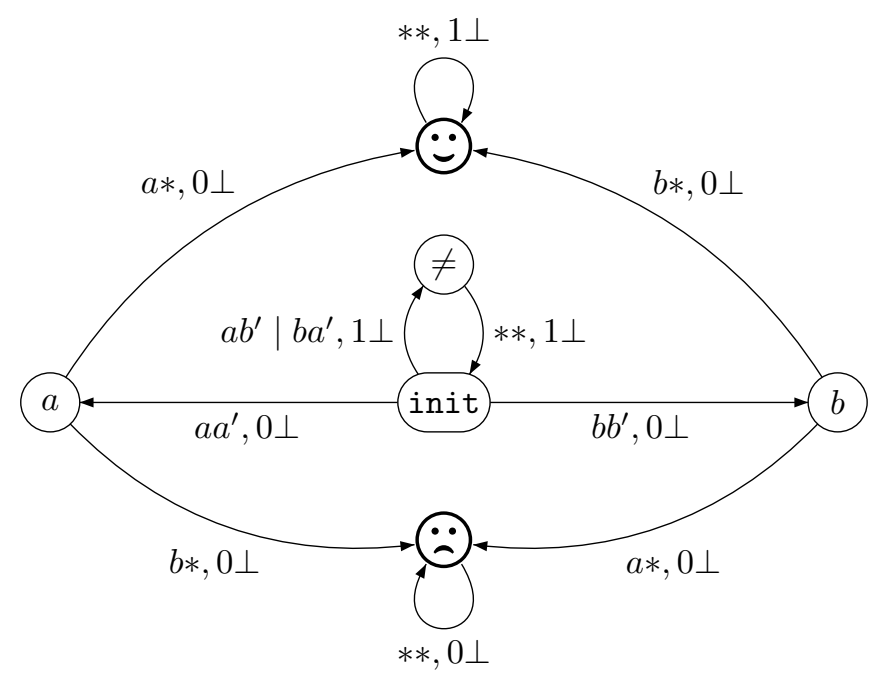

Fig. 2. A two-player stochastic game with signals.

a strategy. In the sequel, we introduce several classes of strategies, defined by the resources available to the players, and discuss how they relate.

\subsection{Finite-memory strategies.}

Since our target application is controller synthesis, we seek strategies which are easily representable and implementable, that is why we are especially interested in finite-memory strategies. In the present paper we study several algorithmic game problems and provide solutions to determine the winner of the game and at the same time compute a finite-memory winning strategy (see Section 6.3.

There are various definitions of finite-memory strategies in the litterature. A quite complete presentation is given in Cristau et al. 2010. We provide now the most general definition of strategies with finite memory, called general finite-memory strategies in Cristau et al. 2010. This is the notion of finite-memory strategy we use throughout the present paper.

A strategy with finite memory set $M$ for player 1 with set of signals $C$ and set of actions $I$ is a tuple $\sigma=\left(\right.$ init, upd, $\left.\sigma_{M}\right)$, with

- init $\in \Delta(M)$ the initial distribution of the memory,

- upd : $C \times M \rightarrow \Delta(M)$ the memory update function,

- $\sigma_{M}: M \rightarrow \Delta(I)$ the action choice.

Note that the memory initialisation, the memory update and the action choice are randomised. Finite-memory strategies for player 2, with set of signals $D$ and set of actions $J$ are defined in a similar way.

A play according to a finite-memory strategy is as follows: the memory is initialised to a memory state $m_{0} \in M$ chosen randomly, according to the probability distribution init. When the memory is in state $m \in M$, the player plays an action according to the distribution $\sigma_{M}(m)$, the transition occurs and the player receives a signal $c$. Then the new memory state is chosen according to the distribution upd $(c, m)$. Note that the memory state of the strategy of a player is not observable by their opponent, the only source of information of a player is the private signals they receive, they have no clue about the strategy structure of their opponent. 
The formal definition of the probability measure on plays generated by a strategy profile, i.e. a strategy for each of the players and an initial distribution, is postponed to subsection 2.3 First we discuss several notions of finite-memory strategies and compare them in terms of expressivity and succinctness.

\subsection{Finite-memory strategies: deterministic or randomised updates?}

We motivate our preference of finite-memory strategies with a randomised update function

$$
\text { upd : } C \times M \rightarrow \Delta(M) \text {, }
$$

which allows the player to perform and store private coin tosses. Another option is to use deterministic update functions:

$$
\text { upd : } C \times M \rightarrow M
$$

as in de Alfaro et al. 2007, Gripon and Serre 2009, Chatterjee and Doyen 2012. We refer to such a strategy as a finite-memory strategy with deterministic updates.

Remark that in Cristau et al. 2010, finite-memory strategies with deterministic updates are called behavioural (finite-memory) strategies. However we prefer to avoid using this terminology because in the context of this paper it may be misleading: the adjective behavioural is traditionally used to qualify strategies with arbitrary memory [Aumann 1964]. Moreover in the next subsection we give an example showing that there are behavioural strategies which can be implemented with finite-memory with randomised updates but which cannot be implemented with a finite-memory strategy with deterministic updates.

The conclusion of Cristau et al. 2010 states both classes of strategies, with deterministic or randomised updates, have "strengths and weaknesses" and the authors "do not favour one over the others". However, in the case of stochastic games with signals and Büchi conditions, it seems to us that randomised updates is the right choice, for two reasons: expressivity and succinctness.

Finite-memory strategies with randomised updates are much more succinct. Of course, with controller synthesis in mind, the fewer memory states, the better: a strategy with a small description is easier to compute and implement as a controller. From this point of view, a very strong point in favour of finite-memory strategies with randomised updates is given in Chatterjee and Doyen 2012. Namely, when a player is restricted to deterministic updates, this may cause in the worst case a dramatic blowup of the memory size required to win almost-surely or positively a reachability game.

Actually, the fact that general finite-memory strategies are more expressive than the ones with deterministic updates is related to the observability of actions, which may look like a tiny detail in the first place, but requires cautious attention, as demonstrated in Cristau et al. 2010 Chatterjee and Doyen 2012. When a player chooses their next action with respect to a probability distribution over their set of actions, should we assume that the player observes the action $a$ actually selected by this lottery?

When players are restricted to finite-memory strategies with deterministic updates, letting players observe or not their actions is a game-changer. First, [Cristau et al. 2010] shows that it may change the winner of the game. Second, Corollary 4.8 and Lemma 6.7 in Chatterjee and Doyen 2012 show that non-elementary many memory states may be necessary for player 1 to win almost-surely a reachability game using deterministic updates. This lower bound holds for games when players cannot observe their actions. However in the present paper we demonstrate that exponential memory is sufficient when actions are observable (Proposition 6.5) and according to Theorem 4.9 the same results holds when actions are not observable.

In contrast, when randomised updates are allowed, observing actions makes no difference: we can assume that players do not observe their actions without changing the winner of the game and with very little impact on the memory size of strategies. The reason is given 
in Lemma 4.8. any strategy $\sigma$ with finite memory $M$ can be easily transformed into an equivalent strategy $\sigma^{\prime}$ with finite memory $M \times I$ (and randomised updates), where the action choice is deterministic (and actually very simple: in state $(m, i)$ the player plays action $i$ ). Since the action choice is deterministic, the player knows exactly which action was played, independently of the signals they receive.

\subsection{General, mixed and behavioural strategies}

There are many stochastic games with signals where finite-memory strategies are not sufficient: Baier et al. 2008 gives an example of a one-player Büchi game where the player is blind (i.e. always receives the same signal), the player can win the game with positive probability but no finite-memory strategy ensures this.

Again, like in the case of finite-memory strategies, there are several notions of strategies with arbitrary memory in the literature and we use the most general one in the present paper.

The three natural classes of strategies for player 1 in a stochastic game where she receives signals in $C$ are defined as follows:

- a behavioural strategy associates with each finite sequence of signals of player 1 a probability distribution over her actions:

$$
\sigma: C^{*} \rightarrow \Delta(I)
$$

In case $\sigma$ is not randomised i.e. when the image of $\sigma$ is always a Dirac distribution, the strategy is said to be pure. .

- a mixed strategy is a probability measure over pure strategies:

$$
\sigma \in \Delta\left(C^{*} \rightarrow I\right)
$$

- a general strategy is a probability measure over behavioural strategies:

$$
\sigma \in \Delta\left(C^{*} \rightarrow \Delta(I)\right)
$$

where $C^{*} \rightarrow I$ denotes the set of functions from $C^{*}$ to $I$ equipped with the product topology of the copies of the discrete set $I$ and $C^{*} \rightarrow \Delta(I)$ is the set of functions from $C^{*}$ to $\Delta(I)$ equipped with the product topology of the copies of the metric space $\Delta(I)$. Clearly behavioural and mixed strategies are contained in the class of general strategies.

To our knowledge the class of general strategies was introduced in Cristau et al. 2010. The notions of mixed strategies and behavioural strategies are classic. Kuhn's theorem states that these classes of strategies are equivalent when players have perfect recall Aumann 1995.

We use $K_{n}, I_{n}, J_{n}, C_{n+1}$ and $D_{n+1}$ to denote the random variables corresponding respectively to $n$-th state, action of player 1 , action of player 2 , signal of player 1 and signal of player 2 and we denote $P_{n}$ the finite play $P_{n}=K_{0}, I_{0}, J_{0}, C_{1}, D_{1}, K_{1}, \ldots, C_{n}, D_{n}, K_{n}$.

In the usual way, an initial distribution $\delta$ and two behavioural strategies $\sigma$ and $\tau$ define a probability measure $\mathbb{P}_{\delta}^{\sigma, \tau}$ on the set of infinite plays, equipped with the $\sigma$-algebra generated by cylinders, that is, sets of infinite plays that extend a common prefix finite play. The probability measure $\mathbb{P}_{\delta}^{\sigma, \tau}$ is the only probability measure over $(K I J C D)^{\omega}$ such that for every $k \in K, \mathbb{P}_{\delta}^{\sigma, \tau}\left(K_{0}=k\right)=\delta(k)$ and for every $n \in \mathbb{N}$,

$$
\begin{aligned}
& \mathbb{P}_{\delta}^{\sigma, \tau}\left(K_{n+1}, C_{n+1}, D_{n+1} \mid P_{n}\right) \\
& \quad=\sigma\left(P_{n}\right)\left(C_{n+1}\right) \cdot \tau\left(P_{n}\right)\left(D_{n+1}\right) \cdot p\left(K_{n+1}, C_{n+1}, D_{n+1} \mid K_{n}, I_{n}, J_{n}\right),
\end{aligned}
$$

where we use standard notations for conditional probability measures.

A pair of general strategies $\Sigma \in \Delta\left(C^{*} \rightarrow \Delta(I)\right)$ for player 1 and $T \in \Delta\left(D^{*} \rightarrow \Delta(J)\right)$ for player 2 and an initial probability distribution $\delta$ define altogether a probability measure 
$\mathbb{P}_{\delta}^{\Sigma, T}$ over the set of infinite plays, for $E \subseteq(K I J C D)^{\omega}$ measurable,

$$
\mathbb{P}_{\delta}^{\Sigma, T}(E)=\int_{\sigma: C^{*} \rightarrow \Delta(I)} \int_{\tau: D^{*} \rightarrow \Delta(J)} \mathbb{P}_{\delta}^{\sigma, \tau}(E) d \Sigma(\sigma) d T(\tau) .
$$

This is well defined since the collection $\mathcal{E}$ of events $E \subseteq(K I J C D)^{\omega}$ such that the function $(\sigma, \tau) \rightarrow \mathbb{P}_{\delta}^{\sigma, \tau}(E)$ is measurable contains all measurable $E$. This is because $\mathcal{E}$ clearly contains cylinders and is stable by complement and countable union.

Actually there is an equivalent way to define $\mathbb{P}_{\delta}^{\Sigma, T}$.

Lemma 2.3. For every general strategy $\Sigma$ of player 1 define $\mathbb{E}_{\Sigma}: I(C I)^{*} \rightarrow[0,1]$ by

$$
\mathbb{E}_{\Sigma}\left(i_{0}, c_{1}, \ldots, c_{n}, i_{n}\right)=\int_{\sigma: C^{*} \rightarrow \Delta(I)} \sigma(\varepsilon)\left(i_{0}\right) \cdot \sigma\left(c_{0}\right)\left(i_{1}\right) \cdots \sigma\left(c_{0} \cdots c_{n}\right)\left(i_{n}\right) d \Sigma(\sigma) .
$$

Then $\mathbb{P}_{\delta}^{\Sigma, T}$ is the only probability measure on the set of infinite plays such that for every finite play $\pi=k_{0}, i_{0}, j_{0}, c_{1}, d_{1}, k_{1}, \ldots k_{n}$,

$$
\mathbb{P}_{\delta}^{\Sigma, T}\left(P_{n}=\pi\right)=\delta\left(k_{0}\right) \cdot \mathbb{E}_{\Sigma}\left(i_{0}, c_{1}, \ldots, c_{n}, i_{n}\right) \cdot \mathbb{E}_{T}\left(j_{0}, d_{1}, \ldots, d_{n}, j_{n}\right) .
$$

Proof. A simple computation shows that the condition is necessary. And this defines a unique probability measure since the events $\left\{P_{n}=\pi\right\}$ are exactly the cylinders of $K(I J C D)^{\omega}$, and these cylinders generate the whole $\sigma$-algebra.

\subsection{From finite-memory to general strategies}

Of course a finite-memory strategy can be seen as a general strategy: intuitively, the state space of the game is enlarged by including the memory state, which is observable only by the player playing the finite-memory strategy, and the memory state is updated upon each transition of the game.

The formal definition of the general strategy $\Sigma_{M}$ associated with a finite-memory strategy $\sigma=\left(M\right.$, init, upd, $\left.\sigma_{M}\right)$ for player 1 requires some care. We use an intermediate object $\mu_{M}$ which describes how memory updates are performed in $\sigma$. Let $\mu_{M}$ the unique probability measure on the set of functions $f: C^{*} \times M \rightarrow M$ equipped with the Borel algebra generated by the product topology and such that

$$
\mu_{M}\left(\left\{f \mid f\left(c_{0} \cdots c_{n}, m\right)=m^{\prime}\right\}\right)=\operatorname{upd}\left(m, c_{n}\right)\left(m^{\prime}\right) .
$$

A fixed $m_{0} \in M$ and $f: C^{*} \times M \rightarrow M$ naturally define a behavioural strategy $\sigma_{M, m_{0}, f}$ : $C^{*} \rightarrow \Delta(I)$ by

$$
\begin{aligned}
& \sigma_{M, m_{0}, f}\left(c_{0} \cdots c_{n}\right)=\sigma_{M}\left(m_{n}\left(c_{0} \cdots c_{n}\right)\right) \text { if } n=0 \\
& \text { where } m_{n}\left(c_{0} \cdots c_{n}\right)= \begin{cases}m_{0} & \text { otherwise. } \\
f\left(c_{0} \cdots c_{n}, m_{n-1}\right)\end{cases}
\end{aligned}
$$

Then the general strategy $\Sigma_{M}$ associated with $\sigma_{M}$ is defined by:

$$
\Sigma_{M}(E)=\sum_{m_{0} \in M} \operatorname{init}\left(m_{0}\right) \cdot \mu_{M}\left(\left\{f \mid \sigma_{M, m_{0}, f} \in E\right\}\right) .
$$

REMARK 2.4. The class of behavioural finite-memory strategies defined in Cristau et al. 2010, which we call finite-memory with deterministic updates in the present paper, does not coincide with the intersection of the set of finite-memory strategies and the set of behavioural strategies. A counter-example is the behavioural strategy $\sigma: C^{*} \rightarrow \Delta(\{a, b\})$ defined by $\sigma\left(c_{1}, \ldots, c_{n}\right)(a)=\sum_{i=1}^{n} \frac{1}{2^{i}}$. This strategy is behavioural by definition, and can easily be implemented by a finite-memory strategy with randomised updates and two memory states $\{A, B\}$ as follows: $\operatorname{init}(B)=1, \sigma_{M}(A)(a)=1$ and $\sigma_{M}(B)(b)=1$, and for 
every $c \in C, \operatorname{upd}(c, B)(A)=\operatorname{upd}(c, B)(B)=\frac{1}{2}$, and $\operatorname{upd}(c, A)(A)=1$. However, since $\sigma\left(c_{1}, \ldots, c_{n}\right)(a)$ can take infinitely many different values, no finite-memory strategy with deterministic updates can implement $\sigma$.

\subsection{Winning conditions and winning strategies.}

The goal of player 1 is described by a measurable set of infinite plays Win called the winning condition. Formally, a game is a pair made of an arena and a winning condition on the arena.

Motivated by applications in logic and controller synthesis Grädel et al. 2002], we are especially interested in reachability, safety, Büchi and co-Büchi conditions. These four winning conditions use a subset $T \subseteq K$ of target states in their definition.

The reachability condition stipulates that $T$ should be visited at least once,

$$
\text { Reach }=\left\{\exists n \in \mathbb{N}, K_{n} \in T\right\} .
$$

The safety condition is dual:

$$
\text { Safe }=\left\{\forall n \in \mathbb{N}, K_{n} \notin T\right\} .
$$

For the Büchi condition the set of target states has to be visited infinitely often,

$$
\text { Büchi }=\left\{\forall m \in \mathbb{N}, \exists n \geq m, K_{n} \in T\right\} .
$$

And the co-Büchi condition is dual:

$$
\text { CoBüchi }=\left\{\exists m \in \mathbb{N}, \forall n \geq m, K_{n} \notin T\right\} .
$$

When player 1 and 2 use strategies $\sigma$ and $\tau$ and the initial distribution is $\delta$, then player 1 wins the game with probability:

$$
\mathbb{P}_{\delta}^{\sigma, \tau}(\text { Win })
$$

Player 1 wants to maximise this probability, while player 2 wants to minimise it. An enjoyable situation for player 1 is when she has an almost-surely winning strategy.

Definition 2.5 (Almost-surely winning strategy). A strategy $\sigma$ for $\quad$ player 1 is almost-surely winning from an initial distribution $\delta$ if

$$
\forall \tau, \mathbb{P}_{\delta}^{\sigma, \tau}(\text { Win })=1
$$

When such an almost-surely strategy $\sigma$ exists, the initial distribution $\delta$ is said to be almostsurely winning (for player 1).

A less enjoyable situation for player 1 is when she only has a positively winning strategy.

Definition 2.6 (Positively winning strategy). A strategy $\sigma$ for player 1 is positively winning from an initial distribution $\delta$ if

$$
\forall \tau, \mathbb{P}_{\delta}^{\sigma, \tau}(\text { Win })>0
$$

When such a strategy $\sigma$ exists, the initial distribution $\delta$ is said to be positively winning (for player 1).

Symmetrically, a strategy $\tau$ for player 2 is positively winning if it guarantees $\forall \sigma, \mathbb{P}_{\delta}^{\sigma, \tau}($ Win $)<1$.

The worst situation for player 1 is when her opponent has an almost-surely winning strategy $\tau$, which thus ensures $\mathbb{P}_{\delta}^{\sigma, \tau}$ (Win) $=0$ for all strategies $\sigma$ chosen by player 1 .

Note that whether a distribution $\delta$ is almost-surely or positively winning depends only on its support, because $\mathbb{P}_{\delta}^{\sigma, \tau}($ Win $)=\sum_{k \in K} \delta(k) \cdot \mathbb{P}_{\delta}^{\sigma, \tau}$ (Win $\left.\mid K_{0}=k\right)$. As a consequence, we will say that a support $L \subseteq K$ is almost-surely or positively winning for a player if there exists a distribution with support $L$ which has the same property. 


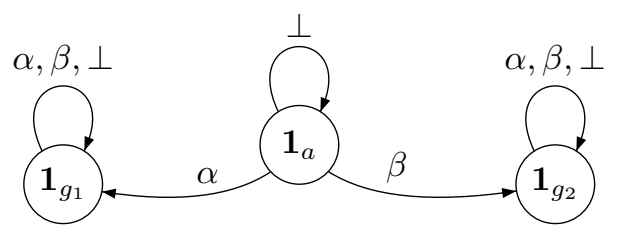

Fig. 3. A three-state almost-surely winning finite-memory strategy for the game of Figure 1 The initial distribution is the Dirac distribution on the middle state. States are labelled by the distribution to be played, all of them are Dirac distributions on this example.

Example 2.7. Consider the one-player game depicted on Fig. 1. The objective of player 1 is to reach the $\odot$-state. The initial distribution is $\delta(1)=\delta(2)=\frac{1}{2}$ and $\delta(\odot)=\delta(\odot)=0$.

In this game, player 1 has a strategy to reach $;$ almost-surely. Her strategy is to keep playing action $a$ as long as she keeps receiving signal $\perp$. The day player 1 receives signal $\alpha$ or $\beta$, she plays respectively action $g_{1}$ or $g_{2}$. This strategy is almost-surely winning because the probability for player 1 to receive signal $\perp$ forever is 0 . This almost-surely winning strategy can be represented by finite-memory strategy with three memory states $M=$ $\left\{m_{a}, m_{1}, m_{2}\right\}$ whose initial mapping is constant equal to the Dirac distribution on $m_{a}$ and whose (deterministic) transitions are depicted on Fig. 3.

\subsection{Qualitative determinacy vs value determinacy.}

If an initial distribution is positively winning for player 1 then by definition it is not almostsurely winning for her opponent player 2. A natural question is whether the converse implication holds.

Definition 2.8 (Qualitative determinacy). A winning condition Win is qualitatively determined if for every stochastic game with signals equipped with Win, every initial distribution is either almost-surely winning for player 1 or positively winning for player 2 .

Qualitative determinacy is similar to, but different from, the usual notion of (value) determinacy which refers to the existence of a value. Actually both qualitative determinacy and value determinacy are formally expressed by a quantifier inversion. On one hand, qualitative determinacy rewrites as:

$$
\left(\forall \sigma \exists \tau \mathbb{P}_{\delta}^{\sigma, \tau}(\mathrm{Win})<1\right) \Longrightarrow\left(\exists \tau \forall \sigma \mathbb{P}_{\delta}^{\sigma, \tau}(\mathrm{Win})<1\right)
$$

On the other hand, the game has a value if:

$$
\sup _{\sigma} \inf _{\tau} \mathbb{P}_{\delta}^{\sigma, \tau}(\text { Win }) \geq \inf _{\tau} \sup _{\sigma} \mathbb{P}_{\delta}^{\sigma, \tau}(\mathrm{Win})
$$

Both the converse implication of the first equation and the converse inequality of the second equation are obvious.

While value determinacy is a classical notion in game theory Shapley 1953, Mertens and Neyman 1982, to our knowledge the notion of qualitative determinacy appeared only recently in the context of omega-regular concurrent games de Alfaro et al. 2007, de Alfaro and Henzinger 2000, BPA games Brázdil et al. 2011] and stochastic games with perfect information Horn 2008. Remark that qualitative determinacy for two-player stochastic full-information parity finitely-branching games is currently an open question [Brázdil et al. 2011 .

The existence of an almost-surely winning strategy ensures that the value of the game is 1 , but the converse is not true, even in one-player games. This is shown in Section 3 by the counter-example on Fig. 6. As a consequence, player 2 may have a positively winning strategy in a game with value 1 . 
A difference between qualitative determinacy and value determinacy is that qualitative determinacy may hold for a winning condition but not for the complementary condition. The present paper provides such an example: Büchi games are qualitatively determined but co-Büchi games are not.

Whether a game is qualitatively determined or not depends on the class of strategies used by the players. With general strategies, or equivalently with mixed strategies, Büchi games are qualitatively determined (Theorem 6.1). However if players are restricted to play behavioural strategies or finite-memory strategies with deterministic updates then in general qualitative determinacy does not hold anymore, as shown by the following example.

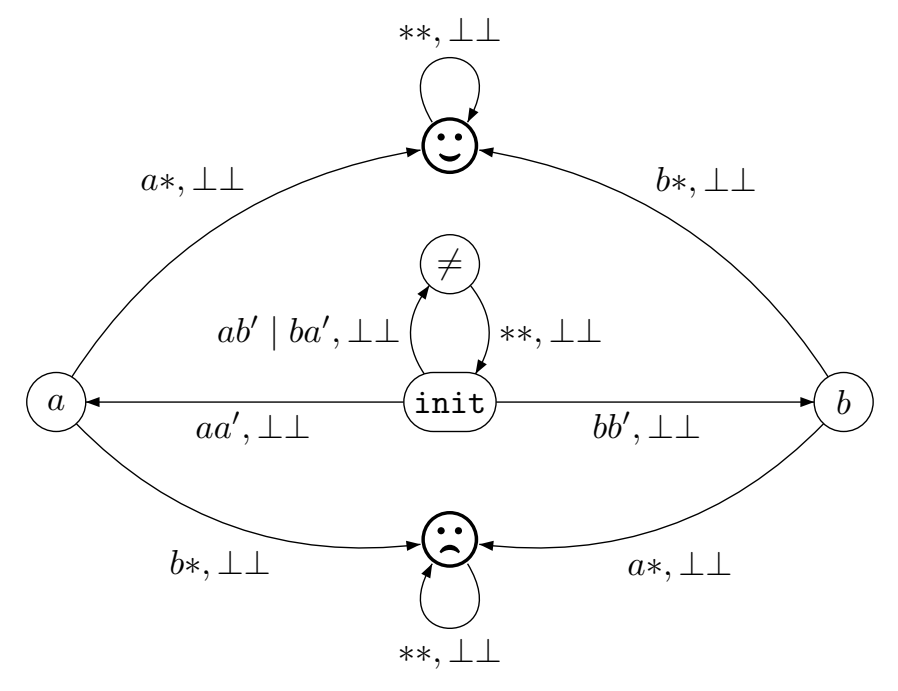

Fig. 4. Example where behavioural strategies are not sufficient.

Example 2.9. Consider the example in Fig. 4 taken from Cristau et al. 2010, where the aim of player 1 is to reach state $\odot$. This example is similar to the one in Fig. 2

Both players are blind: whatever happens, they always receive the same signal $\perp$. Starting in the initial state init, player 1 wishes to reach state $\odot$. For that she has to exit first the set of states $\{$ init,$\neq\}$ by matching the action of her opponent at an even date: $a$ for $a^{\prime}$ and $b$ for $b^{\prime}$. Then she should repeat again the same action in order to reach $\odot$.

Using a behavioural strategy $\sigma: C^{*} \rightarrow \Delta(I)$, player 1 cannot win almost-surely. Since player 1 is blind, $C=\{\perp\}$ and the way player 1 chooses actions only depends upon the time elapsed. There are two cases. First, assume that $\sigma$ plays deterministically at every even time step $2 n$ for every $n \in \mathbb{N}$ (the first step has index 0 ). Then a pure strategy $\tau$ for player 2 beats $\sigma$. It suffices for $\tau$ to play letter $b^{\prime}$ (resp. $a^{\prime}$ ) at step $2 n$ when strategy $\sigma$ plays letter $a$ (resp. $b$ ) at step $2 n$. Then at each odd time point $2 n+1$, the plays is in state $\neq$, and it never reaches state $\odot$. Notice that the actions played at odd time steps are irrelevant. In the second case, consider the first even time point $2 i$ such that both actions $a$ and $b$ are proposed by $\sigma$, with non-zero probability ( $x$ for $a$ and $1-x$ for $b$ ). Then consider what is proposed by $\sigma$ at step $2 i+1$ : by symmetry, assume that $b$ is proposed with non-zero probability $y$ (it is possible that $y=1$, but if $y=0$, we consider letter $a$ instead). Again, because of behavioural strategies, player 1 does not remember her previous actions, nor the actions of player 2 up to now, hence both $a, b$ are proposed whether player 1 played $a$ or played $b$ at step $2 i+1$. 


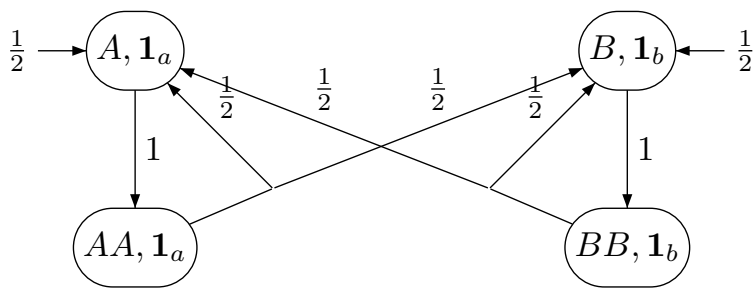

Fig. 5. A four-state almost-surely winning finite-memory strategy for player 1 in the game of Fig. 4 The initial distribution is $\frac{1}{2} A+\frac{1}{2} B$. States are labelled by the distribution to be played, all of them are Dirac distributions on this example: action $a$ in states $A$ and $A A$ and action $b$ in states $B$ and $B B$. There is only one signal $\perp$ for player 1 , which is not represented. The memory updates from $A$ and $B$ are deterministic, those from $A A$ and $B B$ are not.

The strategy $\tau$ of player 2 beating $\sigma$ is the following. It does the opposite of $\sigma$ for the first $i$ even steps (and anything for the first $i$ odd steps - it is irrelevant). Then, it plays deterministically $a^{\prime}$ both at steps $2 i$ and $2 i+1$. At step $2 i$, the play according to $\sigma, \tau$ is in state init with probability 1 . Then with probability $x$, it goes to state $a$, and thus goes to the sink with probability $x y$ after $2 i+1$ steps, and stays there. Hence, the probability to reach $\odot$ under this strategy is at most $1-x y$. That is, behavioural strategies are not sufficient for player 1 to win this game almost-surely.

On the other hand, player 1 has a finite memory strategy $\sigma=\left(\right.$ init, upd, $\sigma_{M}$ ) which is almost-surely winning. The strategy is depicted on Fig. 5. $M$ has 4 states $A, A A, B, B B$, and the initial memory is given by $\operatorname{init}(A)=\operatorname{init}(B)=\frac{1}{2}$. The action choice is deterministic: $\sigma_{M}(A)(a)=\sigma_{M}(A A)(a)=1, \sigma_{M}(B)(b)=\sigma_{M}(B B)(b)=1$. The update function is randomised, defined by upd $(A)(A A)=1, \operatorname{upd}(B)(B B)=1$ and $\operatorname{upd}(A A)(A)=\operatorname{upd}(A A)(B)=$ $\operatorname{upd}(B B)(A)=\operatorname{upd}(B B)(B)=\frac{1}{2}$. It ensures that at odd times, $\{a, b\}$ are played uniformly. Moreover, player 1 knows at every even time point thanks to her memory state what she played at the previous odd time point. Thus, player 1 can play deterministically the same letter. No matter the strategy $\tau$ played by player 2 , the plays following $(\sigma, \tau)$ reach $\odot)$ with probability 1.

Finally, player 1 can win almost-surely with a finite-memory strategy, however no behavioural strategy is almost-surely winning for her: general strategies are more powerful than behavioural strategies.

\section{EXAMPLES}

\subsection{A one-player reachability game with value 1 but player 1 does not win almost-surely}

Consider the one-player game depicted on Fig. 6, which is a slight modification of the one from Fig. 1 (only signals of player 1 and transitions probabilities differ). Player 1 has signals $\{\alpha, \beta\}$ and similarly to the game on Fig. 1, her goal is to reach the target state $\odot$ by guessing correctly whether the initial state is 1 or 2 . On one hand, player 1 can guarantee a winning probability as close to 1 as she wants: she plays $a$ for a long time and compares how often she received signals $\alpha$ and $\beta$. If signal $\alpha$ was more frequent, then she plays action $g_{1}$, otherwise she plays action $g_{2}$. Of course, the longer player 1 plays $a$ 's the more accurate the prediction will be. On the other hand, the only strategy available to player 2 is positively winning, because any sequence of signals in $\{\alpha, \beta\}^{*}$ can be generated with positive probability from both states 1 and 2 . 


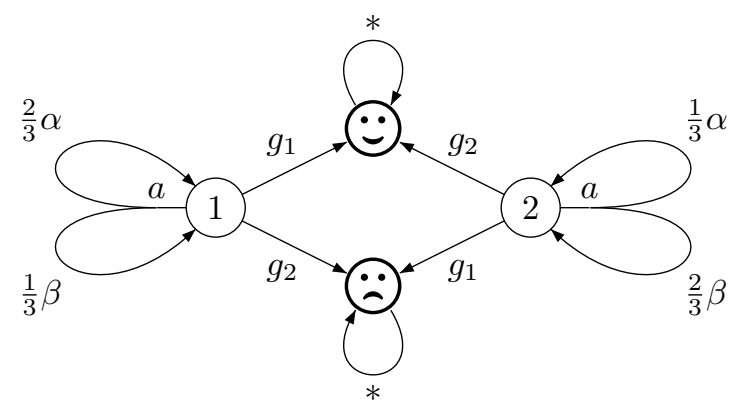

Fig. 6. A one-player reachability game with value 1 where player 1 does not win almost-surely.

\subsection{A game where the signalling structure matters}

We give a second example on Figure 7 where the signalling structure matters, whether player 1 can win positively or not depends not only of her own signalling structure but also of the signalling structure of her opponent.

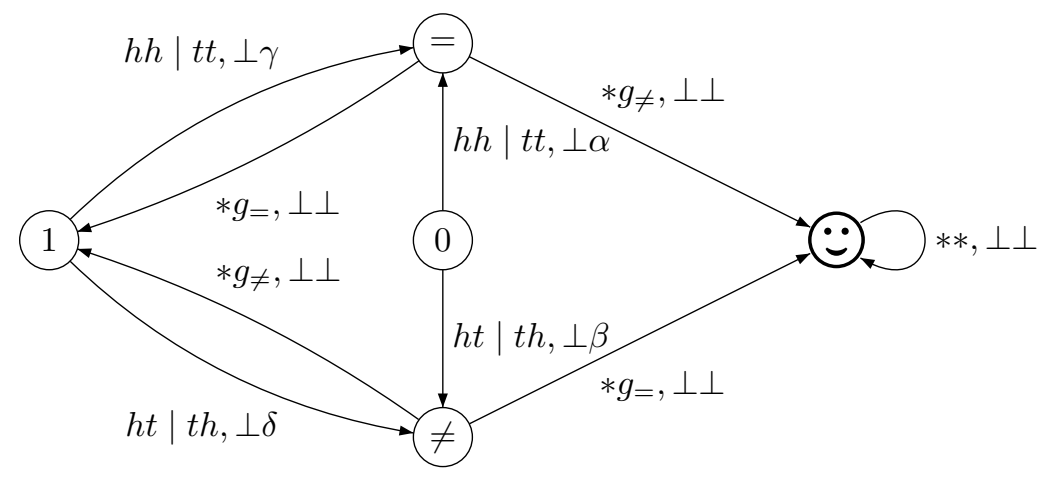

Fig. 7. Player 1 wins almost-surely, positively or not, depending on the signals for player 2 .

The game starts in state 0 , and both players choose heads $(\mathrm{h})$ or tails $(\mathrm{t})$. If they agree the game moves to state $=$, otherwise to state $\neq$. The behaviour is similar from state 1 , but the signals received by player 2 might be different. Player 1 is blind and can only count the

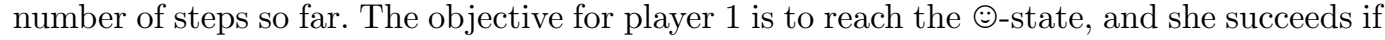
player 2 makes a wrong guess: either he plays $g_{\neq}$from state $e q$ or he plays $g_{=}$from states $\neq$. Depending of the signals $\alpha, \beta, \gamma$ and $\delta$, received by player 2 , the game will be almost-surely winning, positively winning, or winning with probability zero for player 1.

Assume first that all signals $\alpha, \beta, \gamma$ and $\delta$ are distinct. Then, player 2 always knows when the play enters states $e q$ and neq and can play accordingly, in order to avoid the (-)-state. Therefore player 2 has a surely winning strategy (i.e. a strategy such that every play consistent with the strategy is winning for player 2) for her safety objective, and player 1 wins with probability 0 .

Assume now that $\alpha=\beta$, but $\gamma$ and $\delta$ are distinct. Informally, after the first move, player 2 cannot distinguish if the play is in state $=$ or $\neq$. His best choice is then to play uniformly at random $g_{=}$and $g_{\neq}$. Later, if the game reaches state 1 , since $\gamma \neq \delta$, player 2 will be able to avoid the $(-)$-state, whatever player 1 does. For both players, in the first move, the best choice is to play uniformly at random heads or tails, so that in this case, player 1 wins with probability $1 / 2$. 
Last, assume that $\alpha=\beta$ and $\gamma=\delta$, so that player 2 can never distinguish between states $=$ or $\neq$. The best strategy for player 1 is to always choose uniformly at random heads or tails. Against this strategy, and whatever player 2 does, every other move, the probability is half to move to the - -state, so that player 1 wins almost-surely.

\section{GAMES WITH GENERAL STRATEGIES AND NON-OBSERVABLE ACTIONS ARE ALGORITHMICALLY EQUIVALENT TO GAMES WITH BEHAVIOURAL STRATEGIES AND OBSERVABLE ACTIONS.}

In this section we show some connections between general and behavioural strategies, and games with observable and non-observable actions. We show that games with general strategies and non-observable actions are essentially the same as games with behavioural strategies and observable actions. As a consequence, solving games with general strategies and non-observable actions is of the same algorithmic complexity up to linear time reductions as solving games with behavioural strategies and observable actions.

\subsection{Arenas with observable actions}

In general, players may ignore what actions they exactly played at the previous steps, because the signals they receive may not contain this information. Otherwise, the arena they play in is said to have observable actions, in the following sense.

Definition 4.1 (Observable actions). An arena $\mathcal{A}=(K, I, J, C, D, p)$ has observable actions if there exist two mappings Act $: C \rightarrow I$ and Act $2: D \rightarrow J$ such that

$$
p(t, c, d \mid s, i, j)>0 \Longleftrightarrow\left(i=\operatorname{Act}_{1}(c) \wedge j=\operatorname{Act}_{2}(d)\right) .
$$

The action-observable arena associated with an arena $\mathcal{A}=(K, I, J, C, D, p)$ is the arena where actions are added to signals. Formally, this is the arena $\operatorname{Obs}(\mathcal{A})=(K, I, J, C \times I, D \times$ $\left.J, p^{\prime}\right)$ such that $p^{\prime}\left(t,\left(c, i^{\prime}\right),\left(d, j^{\prime}\right) \mid s, i, j\right)=0$ whenever $i \neq i^{\prime}$ or $j \neq j^{\prime}$ and $p^{\prime}(t,(c, i),(d, j) \mid$ $s, i, j)=p(t, c, d \mid s, i, j)$. A strategy $\sigma$ in $\mathcal{A}$ can be naturally seen as a strategy $\operatorname{Obs}(\sigma)$ in $\operatorname{Obs}(\mathcal{A})$ as well, by composition with the projection from $(C \times I)^{*}$ to $C^{*}$. In the same way, a finite or infinite play $\pi=k_{0}, i_{0}, j_{0}, c_{1}, d_{1}, k_{1} \ldots$ in $\mathcal{A}$ can be naturally transformed into the play $\operatorname{Obs}(\pi)=k_{0}, i_{0}, j_{0},\left(c_{1}, i_{0}\right),\left(d_{1}, j_{0}\right), k_{1} \ldots$ in $\operatorname{Obs}(\mathcal{A})$ by adding actions to signals. This defines also a transformation of a winning condition Win in $\mathcal{A}$ to the winning condition $\operatorname{Obs}($ Win $)$ in $\operatorname{Obs}(\mathcal{A})$. This transformation preserves probability measures:

Lemma 4.2. Let $\mathcal{A}$ be an arena. For every general strategies $\Sigma$ and $T$ in $\mathcal{A}$, $\mathbb{P}_{\delta}^{\Sigma, T}(\mathrm{Win})=\mathbb{P}_{\delta}^{\mathrm{Obs}(\Sigma), \operatorname{Obs}(T)}(\mathrm{Obs}(\mathrm{Win}))$.

Proof. Since $\operatorname{Obs}(\Sigma)$ and $\operatorname{Obs}(T)$ do not take into account the actions added to signals in $\operatorname{Obs}(G)$, a finite play $\pi$ has exactly the same probability to occur in $G$ with strategies $\Sigma$ and $T$ than the corresponding finite play $\operatorname{Obs}(\pi)$ in $\operatorname{Obs}(G)$ with strategies $\operatorname{Obs}(\Sigma)$ and $\operatorname{Obs}(T)$. Let $\mathcal{E}$ be the collection of measurable sets of infinite plays $E$ such that $\operatorname{Obs}(E)$ is measurable and $\mathbb{P}_{\delta}^{\Sigma, T}(E)=\mathbb{P}_{\delta}^{\operatorname{Obs}(\Sigma), \operatorname{Obs}(T)}(\operatorname{Obs}(E))$. Then according to supra, $\mathcal{E}$ contains all cylinders. Since $\mathcal{E}$ is closed under complementation and countable union, it contains also all measurable sets, including Win in particular.

\subsection{Preliminary lemmas}

The technical core of our reduction from games with non-observable actions and general strategies to games with observable actions and behavioural strategies is a series of lemmas. Most of these results rely on the notion of equivalent strategies.

Definition 4.3 (Equivalent strategies). In an arena $\mathcal{A}$, two general strategies $\Sigma_{1}, \Sigma_{2}$ for player 1 are equivalent, denoted $\Sigma_{1} \equiv \Sigma_{2}$, if for every general strategy $T$ of player 2 , and every initial distribution $\delta$, the probability measures $\mathbb{P}_{\delta}^{\Sigma_{1}, T}$ and $\mathbb{P}_{\delta}^{\Sigma_{2}, T}$ coincide. 
A sufficient condition for two general strategies to be equivalent is given by the following corollary of Lemma 2.3 .

Lemma 4.4. Two general strategies $\Sigma_{1}, \Sigma_{2}$ are equivalent whenever $\mathbb{E}_{\Sigma_{1}}=\mathbb{E}_{\Sigma_{2}}$.

First we show that mixed strategies are as powerful as general strategies (Lemma 4.5). Then, in case actions are observable, Lemma 4.6 shows that behavioural strategies are as powerful as general strategies. Finally Lemma 4.7 and Lemma 4.8 shows that whether actions are observable or not does not matter when playing with general strategies and finite-memory strategies.

Lemma 4.5. In every arena, every general strategy has an equivalent mixed strategy.

Proof. The idea behind the proof of Lemma 4.5 is very natural. The main difference between a general strategy and a mixed one is that a mixed strategy performs all the randomization it needs once for all before the play begins: once a pure strategy $\sigma: C^{*} \rightarrow I$ is selected, the player can play deterministically. By contrast a general strategy selects a behavioural strategy $\sigma: C^{*} \rightarrow \Delta(I)$ and the player has to use extra random generators during the play in order to play $\sigma$.

Intuitively, it is quite easy for a mixed strategy $\Sigma^{\prime}$ to mimic a general strategy $\Sigma$. Before the play begins, the mixed strategy $\Sigma^{\prime}$ selects a behavioural strategy $\sigma: C^{*} \rightarrow \Delta(I)$ using the lottery $\Sigma$. Moreover, $\Sigma^{\prime}$ resolves every possible future random choices of $\sigma$ by picking uniformly at random a sample $\omega$ in the sample space

$$
\Omega=\left\{C^{*} \rightarrow[0,1]\right\} .
$$

For that we equip $\Omega$ with the uniform probability measure $\mu$ obtained as the product of copies of the uniform measure $\lambda$ on $[0,1]$.

Knowing the sample $\omega \in \Omega$, all future choices can be made deterministically, thanks to a transformation which turns a behavioural strategy $\sigma \in C^{*} \rightarrow \Delta(I)$ and a sample $\omega \in \Omega$ into a pure strategy $\sigma_{\omega} \in C^{*} \rightarrow I$ called the $\omega$-determinization of $\sigma$. To play $\sigma_{\omega}$, when a sequence of signals $c_{0} c_{1} \cdots c_{n}$ has occurred, player 1 does not use the lottery $\sigma\left(c_{0} c_{1} \cdots c_{n}\right) \in \Delta(I)$ to choose her next action. Instead she uses the value of the random sample $\omega\left(c_{0} c_{1} \cdots c_{n}\right)$ to determine this action. This should be done in a way which guarantees that the probability to choose action $i$ is equal to $\sigma\left(c_{0} c_{1} \cdots c_{n}\right)(i)$, i.e. we want the transformation $(\sigma, \omega) \rightarrow \sigma_{\omega}$ to guarantee

$$
\sigma\left(c_{0} \cdots c_{n}\right)(i)=\mu\left(\left\{\omega \mid \sigma_{\omega}\left(c_{0} \cdots c_{n}\right)=i\right\}\right) .
$$

For that, we enumerate $I$ as $I=\left\{i_{0}, i_{1}, \ldots, i_{m}\right\}$ and for every $c_{0} c_{1} \cdots c_{n} \in C^{*}$ we partition $[0,1]$ into $m+1$ intervals $\left[0=x_{0}, x_{1}\left[,\left[x_{1}, x_{2}\left[, \ldots,\left[x_{m-1}, x_{m}\right],\left[x_{m}, x_{m+1}=1\right]\right.\right.\right.\right.$ such that the width of $\left[x_{k}, x_{k+1}\left[\right.\right.$ is proportional to the probability that $\sigma\left(c_{0} \cdots c_{n}\right)$ chooses $i_{k}$ i.e. $x_{k+1}=x_{k}+\sigma\left(c_{0} \cdots c_{n}\right)\left(i_{k}\right)$. This way (5) holds because

$$
\mu\left(\left\{\omega \mid \sigma_{\omega}\left(c_{0} \cdots c_{n}\right)=i_{k}\right\}\right)=\lambda\left(\left[x_{k}, x_{k+1}[)=\sigma\left(c_{0} \cdots c_{n}\right)\left(i_{k}\right) .\right.\right.
$$

Then for every general strategy $\Sigma \in \Delta\left(C^{*} \rightarrow \Delta(I)\right)$ we define a mixed strategy $\Sigma^{\prime} \in$ $\Delta\left(C^{*} \rightarrow I\right)$ as:

$$
\Sigma^{\prime}(E)=\int_{\omega \in \Omega} \Sigma\left(\left\{\sigma \mid \sigma_{\omega} \in E\right\}\right) d \mu(\omega) .
$$

To prove that $\Sigma^{\prime}$ is well defined we have to establish that the function $\Psi_{E}: \omega \rightarrow \Sigma(\{\sigma \mid$ $\left.\left.\sigma_{\omega} \in E\right\}\right)$ is measurable whenever $E$ is. Remark that for every $c_{0} \cdots \in C^{*}$ and $i \in I$ the function from $\phi:[0,1] \rightarrow[0,1]$ defined by $x \rightarrow \Sigma\left(\sigma \mid \sigma\left(c_{0} \cdots c_{n}\right)(i) \geq x\right)$ is monotonic thus it is Lebesgue-measurable. If $E=\left\{\sigma \mid \sigma\left(c_{0} \cdots c_{n}\right)=i\right\}$ then

$$
\Psi_{E}(\omega)=\Sigma\left(\left\{\sigma \mid \sigma_{\omega} \in E\right\}\right)=\Sigma\left(\left\{\sigma \mid \sigma\left(c_{0} \cdots c_{n}\right)(i) \geq \omega\left(c_{0} \cdots c_{n}\right)\right\}\right)=\phi\left(\omega\left(c_{0} \cdots c_{n}\right)\right)
$$


thus $\Psi_{E}$ is Lebesgue-measurable whenever $E$ is a cylinder. Moreover the class of $E$ such that $\Psi_{E}$ is measurable is stable by complement and countable unions. Thus $\Psi_{E}$ is well-defined.

To show that $\Sigma$ and $\Sigma^{\prime}$ are equivalent we rely on Lemma 4.4

$$
\begin{aligned}
& \mathbb{E}_{\Sigma}\left(i_{0}, c_{1}, \ldots, c_{n}, i_{n}\right)=\int_{\sigma: C^{*} \rightarrow \Delta(I)} \sigma(\varepsilon)\left(i_{0}\right) \cdot \sigma\left(c_{0}\right)\left(i_{1}\right) \cdots \sigma\left(c_{0} \cdots c_{n}\right)\left(i_{n}\right) d \Sigma(\sigma) \\
= & \int_{\sigma: C^{*} \rightarrow I} \int_{\omega \in \Omega} \mu\left(\left\{\omega \mid \sigma_{\omega}(\varepsilon)=i_{0}, \sigma_{\omega}\left(c_{1}\right)=i_{0}, \ldots, \sigma_{\omega}\left(c_{1} \cdots c_{n}\right)=i_{n}\right\}\right) d \mu(\omega) d \Sigma(\sigma) \\
= & \int_{\omega \in \Omega} \Sigma\left(\left\{\sigma: C^{*} \rightarrow I \mid \sigma_{\omega}(\varepsilon)=i_{0}, \sigma_{\omega}\left(c_{1}\right)=i_{0}, \ldots, \sigma_{\omega}\left(c_{1} \cdots c_{n}\right)=i_{n}\right\}\right) d \mu(\omega) \\
= & \Sigma^{\prime}\left(\left\{\sigma: C^{*} \rightarrow I \mid \sigma(\varepsilon)=i_{0}, \sigma\left(c_{1}\right)=i_{0}, \ldots, \sigma\left(c_{1} \cdots c_{n}\right)=i_{n}\right\}\right) \\
= & \mathbb{E}_{\Sigma^{\prime}}\left(i_{0}, c_{1}, \ldots, c_{n}, i_{n}\right),
\end{aligned}
$$

where the first and last inequalities are by definition of $\mathbb{E}_{\Sigma}$ and $\mathbb{E}_{\Sigma^{\prime}}$, the second equality is a consequence of (5), the third is Fubini's theorem and the fourth is the definition of $\Sigma^{\prime}$.

The following result is a corollary of the generalization of Kuhn's theorem proved in $\mathrm{Au}-$ mann 1995]: whenever players have perfect recall, mixed strategies and behavioural strategies are equivalent. In order for this section to be self-contained, we provide a proof along the same lines.

LEMMA 4.6. In every arena with observable actions, every general strategy has an equivalent behavioural strategy.

Proof. Let $\mathcal{A}$ be an arena with observable actions. Thanks to Lemma 4.5, we can assume without loss of generality that $\Sigma$ is a mixed strategy i.e. $\Sigma \in \Delta\left(C^{*} \rightarrow I\right)$. For a sequence $c_{1} \cdots c_{k}$ of signals, possibly empty when $k=0$, we define the set $E\left(c_{1} \cdots c_{k}\right)$ of pure strategies which are consistent with the actions associated to signals $c_{1} \cdots c_{k}$ :

$$
E\left(c_{1} \cdots c_{k}\right)=\left\{\sigma: C^{*} \rightarrow I \mid \sigma(\varepsilon)=\operatorname{Obs}\left(c_{1}\right) \wedge \forall 1 \leq k \leq n-1, \sigma\left(c_{1} \cdots c_{k}\right)=\operatorname{Act}_{1}\left(c_{k+1}\right)\right\},
$$

and for $i \in I, E\left(c_{1} \cdots c_{k}, i\right)=\left\{\sigma \in E\left(c_{1} \cdots c_{k}\right) \mid \sigma\left(c_{1} \cdots c_{k}\right)=i\right\}$. Then let $\sigma_{b}$ the behavioural strategy in $\mathcal{A}$ defined for $c_{1} \cdots c_{n} \in C^{*}$ and $i \in I$ by $\sigma_{b}\left(c_{1} \cdots c_{n}\right)(i)=$ $\Sigma\left(E\left(c_{1} \cdots c_{n}, i\right) \mid E\left(c_{1} \cdots c_{n}\right)\right)$. By definition of $\mathbb{E}_{\Sigma}$, this guarantees $\mathbb{E}_{\Sigma}=\mathbb{E}_{\sigma_{b}}$. According to Lemma 4.4 the strategies $\sigma_{b}$ and $\Sigma$ are equivalent.

Remark that Lemma 4.6 does not hold if actions are not observable, a counter-example inspired from Cristau et al. 2010, is given in Fig. 4 in the examples section (Section 3). The observability of actions is crucial in the proof of Lemma 4.6. For example assume that $\Sigma$ is a general strategy which selects with equal probability $\frac{1}{2}$ the two pure strategies which play always $i_{0}$ or always $i_{1}$. Then the behavioural strategy $\sigma_{b}$ constructed by the proof selects randomly the first action and then repeats it forever, which is equivalent to $\Sigma$. Playing $\sigma_{b}$ is possible only if actions are observable. In case actions are not observable, it is natural to consider the behavioural strategy $\sigma_{b}^{\prime}$

$$
\sigma_{b}^{\prime}\left(c_{1} \cdots c_{n}\right)(i)=\int_{\sigma: C^{*} \rightarrow \Delta(I)} \sigma\left(c_{1} \cdots c_{n}\right)(i) d \Sigma(\sigma) .
$$

However there is no guarantee that $\sigma_{b}^{\prime}$ and $\Sigma$ are equivalent. Using the same example, $\sigma_{b}^{\prime}$ is the strategy which always plays the lottery $\frac{1}{2} i_{0}+\frac{1}{2} i_{1}$. Clearly, $\sigma_{b}^{\prime}$ and $\Sigma$ are not equivalent: $\sigma_{b}^{\prime}$ plays almost-surely infinitely many times both actions $i_{0}$ and $i_{1}$ while this never happens when playing $\Sigma$.

LEMMA 4.7. For every general strategy $\Sigma$ in $\operatorname{Obs}(\mathcal{A})$ there exists a general strategy $\Sigma^{\prime}$ in $\mathcal{A}$ such that $\Sigma$ is equivalent to $\operatorname{Obs}\left(\Sigma^{\prime}\right)$. 
Proof. Thanks to Lemma 4.5, we can assume without loss of generality that $\Sigma$ is a mixed strategy.

We start with the even simpler case where $\Sigma$ is the Dirac distribution on a single pure strategy $\sigma:(C \times I)^{*} \rightarrow I$ in $\operatorname{Obs}(\mathcal{A})$. This case is easy since a player playing the pure strategy $\sigma$ can use the definition of $\sigma$ to compute their past actions, and thus the player can forget the actions included in the signals. Formally, we define a pure strategy $\sigma_{f}$ in $\mathcal{A}$ by $\sigma_{f}(\varepsilon)=\sigma(\varepsilon)$ and the inductive formula $\sigma_{f}\left(c_{1} \cdots c_{n}\right)=\sigma\left(\left(c_{1}, \sigma_{f}(\varepsilon)\right) \cdot\left(c_{2}, \sigma_{f}\left(c_{1}\right)\right) \cdots\left(c_{n}, \sigma_{f}\left(c_{1} \cdots c_{n-1}\right)\right)\right.$. Then clearly $\operatorname{Obs}\left(\sigma_{f}\right)=\sigma$. Then a sequence of signals $c_{1} \cdots c_{n}$ is consistent with $\sigma$ (in sense that $\left.\forall k, \sigma\left(c_{1} \cdots c_{k-1}\right)=\operatorname{Act}_{1}\left(c_{k}\right)\right)$ if and only if $c_{1} \cdots c_{n}$ is consistent with $\operatorname{Obs}\left(\sigma_{f}\right)$. As a consequence $\mathbb{E}_{\sigma}=\mathbb{E}_{\mathrm{Obs}\left(\sigma_{f}\right)}$ thus $\sigma$ and $\operatorname{Obs}\left(\sigma_{f}\right)$ are equivalent according to Lemma 4.4 .

Assume now that $\Sigma$ is a mixed strategy in $\operatorname{Obs}(\mathcal{A})$ and let $\Sigma^{\prime}$ be the mixed strategy in $\mathcal{A}$ defined for $E \subseteq C^{*} \rightarrow I$ by $\Sigma^{\prime}(E)=\Sigma\left(\left\{\sigma \mid \sigma_{f} \in E\right\}\right)$. According to Lemma 4.4 it is enough to prove $\mathbb{E}_{\Sigma}=\mathbb{E}_{\mathrm{Obs}\left(\Sigma^{\prime}\right)}$. This holds because for every sequence $u=\left(i_{0},\left(c_{1}, i_{0}\right), \ldots,\left(c_{n}, i_{n-1}\right), i_{n}\right) \in I((C \times I) \times I)^{n-1}, \mathbb{E}_{\Sigma}(u)=\int_{\sigma:(C \times I)^{*} \rightarrow I} \mathbb{E}_{\sigma}(u) d \Sigma(\sigma)=$ $\int_{\sigma:(C \times I)^{*} \rightarrow I} \mathbb{E}_{\mathrm{Obs}\left(\sigma_{f}\right)}(u) d \Sigma(\sigma)=\int_{\sigma_{f}: C^{*} \rightarrow I} \mathbb{E}_{\mathrm{Obs}\left(\sigma_{f}\right)}(u) d \Sigma^{\prime}\left(\sigma_{f}\right)=\mathbb{E}_{\mathrm{Obs}\left(\Sigma^{\prime}\right)}(u)$ where the first equality holds by definition of $\mathbb{E}_{\Sigma}$ in case $\Sigma$ is a mixed strategy, the second because we proved already $\mathbb{E}_{\sigma}=\mathbb{E}_{\mathrm{Obs}\left(\sigma_{f}\right)}$, the third and fourth by definition of $\Sigma^{\prime}$ and $\mathbb{E}_{\Sigma^{\prime}}$.

Lemma 4.8. For every finite-memory strategy $\sigma$ with memory $M$ in $\operatorname{Obs}(\mathcal{A})$, there exists a finite-memory strategy $\sigma^{\prime}$ with memory $M \times I$ in $\mathcal{A}$, such that $\sigma$ and $\operatorname{Obs}\left(\sigma^{\prime}\right)$ are equivalent. Moreover the action choice of $\sigma^{\prime}$ is simply the projection of $M \times I$ to $I$.

Proof. From $\sigma=\left(\right.$ init, upd, $\left.\sigma_{M}\right)$ on $M$ we define $\sigma^{\prime}=\left(\right.$ init $^{\prime}$, upd $\left.^{\prime}, \sigma_{M}^{\prime}\right)$ on $M \times I$ where we encode action choices in the set of memory states: in $\sigma^{\prime}$, each transition not only performs the corresponding transition of $\sigma$ to the next memory state $m^{\prime}$ but also simultaneously selects the next action to be played, according to the distribution $\sigma\left(m^{\prime}\right)$. Formally, the action choice of $\sigma^{\prime}$ is the projection $\sigma_{M}^{\prime}(m, i)=i$, the memory update is $\operatorname{upd}^{\prime}\left((m, i), c,\left(m^{\prime}, i^{\prime}\right)=\right.$ $\operatorname{upd}\left(m, c, m^{\prime}\right) \cdot \sigma_{M}\left(m^{\prime}\right)\left(i^{\prime}\right)$ and the initial memory choice is $\operatorname{init}^{\prime}(m, i)=\operatorname{init}(m) \cdot \sigma_{M}(m, i)$. This terminates the proof of Lemma 4.8 .

In general the transformation of Lemma 4.8 does not preserve deterministic updates:

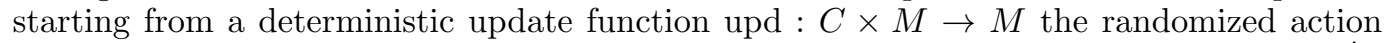
choice $\sigma_{M}: M \rightarrow \Delta(I)$ is integrated into the new (randomized) update function upd' : $C \times(M \times I) \rightarrow \Delta(M \times I)$. To guarantee that the resulting strategy has deterministic updates, we need both the update and the action choice to be deterministic.

\subsection{Equivalence of games with general strategies and games with observable actions and behavioural strategies}

We combine the results obtained so far to prove that a player has a winning general strategy in a game if and only if they have a winning behavioural strategy in the variant of the same game where actions are included in signals and thus become observable.

TheOREm 4.9. Let $\mathcal{A}$ be an arena and $\operatorname{Obs}(\mathcal{A})$ the action-observable arena associated with $\mathcal{A}$. Let Win be a winning condition on $\mathcal{A}$ and consider the two games $G=(\mathcal{A}$, Win $)$ and $\operatorname{Obs}(G)=(\operatorname{Obs}(\mathcal{A}), \operatorname{Obs}($ Win $))$. Then the three following statements are equivalent.

i) Player 1 wins $G$ almost-surely,

ii) Player 1 wins $\operatorname{Obs}(G)$ almost-surely,

iii) Player 1 has a behavioural strategy in $\operatorname{Obs}(G)$ which is almost-surely winning.

The same equivalence holds if ones replaces "almost-surely" by "positively" and/or "player 1 " by "player 2". 
Moreover, every almost-surely (resp. positively) winning finite-memory strategy in $\operatorname{Obs}(G)$ can be turned in linear time into an almost-surely (resp. positively) winning finite-memory strategy in $G$.

Proof. First, iii) and ii) are equivalent because iii) implies ii) trivially and Lemma 4.6 shows that ii) implies iii).

Now we prove that i) and ii) are equivalent. Assume player 1 has an almost-surely winning strategy $\Sigma$ for $G$, and let us prove that $\operatorname{Obs}(\Sigma)$ is almost-surely winning in $\operatorname{Obs}(G)$. Let $T$ be a strategy in $\operatorname{Obs}(G)$ and $T^{\prime}$ the strategy in $G$ given by Lemma 4.7, such that $\operatorname{Obs}\left(T^{\prime}\right)$ is equivalent to $T$. Then:

$$
\mathbb{P}_{\delta}^{\mathrm{Obs}(\Sigma), T}(\operatorname{Obs}(\mathrm{Win}))=\mathbb{P}_{\delta}^{\mathrm{Obs}(\Sigma), \operatorname{Obs}\left(T^{\prime}\right)}(\operatorname{Obs}(\mathrm{Win}))=\mathbb{P}_{\delta}^{\Sigma, T^{\prime}}(\mathrm{Win})=1
$$

where the first equality is by choice of $T^{\prime}$, the second equality is Lemma 4.2 and the third equality is because $\Sigma$ is almost-surely winning in $\mathcal{A}$. It proves that $\operatorname{Obs}(\Sigma)$ is almost-surely winning in $\operatorname{Obs}(G)$.

Last, assume ii) holds and let us prove i). Let $\Sigma$ be a strategy of player 1 winning almost-surely in $\operatorname{Obs}(G)$. According to Lemma 4.7 there exists a strategy $\Sigma^{\prime}$ in $G$ such that $\operatorname{Obs}\left(\Sigma^{\prime}\right) \equiv \Sigma$. Let us prove that $\Sigma^{\prime}$ is almost-surely winning in $G$. For any strategy $T$ of player 2 in $G$,

$$
\mathbb{P}_{\delta}^{\Sigma^{\prime}, T}(\text { Win })=\mathbb{P}_{\delta}^{\operatorname{Obs}\left(\Sigma^{\prime}\right), \operatorname{Obs}(T)}(\operatorname{Obs}(\text { Win }))=\mathbb{P}_{\delta}^{\Sigma, \operatorname{Obs}(T)}(\text { Win })=1 .
$$

Indeed, the first equality is Lemma 4.2 , the second is by choice of $\Sigma^{\prime}$ and the last because $\Sigma$ is almost-surely winning in $\operatorname{Obs}(G)$. Thus i), ii) and iii) are equivalent.

The last statement about finite-memory strategies is a consequence of Lemma 4.8 .

Theorem 4.9 has an algorithmic corollary: every algorithm which decides the existence of an almost-surely winning strategy in games with behavioural strategies and observable actions can be used to decide the same problem in games with general strategies and nonobservable actions. For that it suffices to compute the action-observable version of the game, as defined below, and Theorem 4.9 ensures that this transformation has no incidence on the winner and that winning finite-memory strategies in the observable game can be lifted to winning finite-memory strategies in the original game.

This reduction leaves open an algorithmic question, which is not addressed in the present paper: in case players cannot observe their actions, is it possible to decide the existence of an almost-surely or a positively winning behavioural strategy?

\section{BELIEF STRATEGIES}

Beliefs and beliefs of beliefs formalise part of the knowledge of players during the game. They are used to define belief strategies, which are finite-memory strategies of particular interest. For these notions to be properly defined, the arena should have observable actions (in the sense of Definition 4.1.

\subsection{Beliefs and 2-beliefs}

The belief of a player is the set of possible states of the game, according to the signals received by the player.

Definition 5.1 (Belief). Let $\mathcal{A}$ be an arena with observable actions. From an initial set of states $L \subseteq K$, the belief of player 1 after having received signal $c$ is:

$$
\mathcal{B}_{1}(L, c)=\left\{k \in K \mid \exists l \in L, d \in D \text { such that } p\left(k, c, d \mid l, \operatorname{Act}_{1}(c), \operatorname{Act}_{2}(d)\right)>0\right\} .
$$

Remark that in this definition we use the fact that actions of player 1 are observable, thus when he receives a signal $c \in C$ player 1 can deduce he played action $\operatorname{act}_{1}(c) \in I$. 
The belief of player 1 after having received a sequence of signals $c_{1}, \ldots, c_{n}$ is defined inductively by:

$$
\mathcal{B}_{1}\left(L, c_{1}, c_{2}, \ldots, c_{n}\right)=\mathcal{B}_{1}\left(\mathcal{B}_{1}\left(L, c_{1}, \ldots, c_{n-1}\right), c_{n}\right) .
$$

Beliefs of player 2 are defined similarly. Given an initial distribution $\delta$, we denote $\mathcal{B}_{1}^{n}$ the random variable defined by

$$
\begin{aligned}
& \mathcal{B}_{1}^{0}=\operatorname{supp}(\delta) \\
& \mathcal{B}_{1}^{n+1}=\mathcal{B}_{1}\left(\operatorname{supp}(\delta), C_{1}, \ldots, C_{n+1}\right)=\mathcal{B}_{1}\left(\mathcal{B}_{1}^{n}, C_{n+1}\right) .
\end{aligned}
$$

We will also rely on the notion of belief of belief, called here 2-belief, which, roughly speaking, represents for one player the set of possible beliefs for his (or her) adversary, as well as the possible current state.

Definition 5.2 (2-Belief). Let $\mathcal{A}$ be an arena with observable actions. From an initial set $\mathcal{L} \subseteq K \times \mathcal{P}(K)$ of pairs composed of a state and a belief for player 2, the 2-belief of player 1 after having received signal $c$ is the subset of $K \times \mathcal{P}(K)$ defined by:

$$
\mathcal{B}_{1}^{(2)}(\mathcal{L}, c)=\left\{\left(k, \mathcal{B}_{2}(L, d)\right) \mid(l, L) \in \mathcal{L}, d \in D, p\left(k, c, d \mid l, \operatorname{Act}_{1}(i), \operatorname{Act}_{2}(j)\right)>0\right\} .
$$

From an initial set $\mathcal{L} \subseteq K \times \mathcal{P}(K)$ of pairs composed of a state and a belief for player 2 , the 2-belief of player 1 after having received a sequence of signals $c_{1}, \ldots, c_{n}$ is defined inductively by:

$$
\mathcal{B}_{1}^{(2)}\left(\mathcal{L}, c_{1}, c_{2}, \ldots, c_{n}\right)=\mathcal{B}_{1}^{(2)}\left(\mathcal{B}_{1}^{(2)}\left(\mathcal{L}, c_{1}, \ldots, c_{n-1}\right), c_{n}\right) .
$$

There are natural definitions of 3-beliefs (beliefs on beliefs on beliefs) and even $k$-beliefs however in the present paper we show that 2-beliefs are enough, in some sense: in Büchi games the positively winning sets of player 2 can be characterised by fix-point equations on sets of 2-beliefs, and some positively winning strategies of player 2 with finite-memory can be implemented using 2-beliefs.

\subsection{Belief strategies}

Based on the notions of belief and 2-beliefs, we introduce the following families of strategies with finite-memory, that will be sufficient to win stochastic games with signals either positively or almost-surely.

Definition 5.3 (Belief strategies and 2-belief strategies). Let $\mathcal{A}$ be an arena with observable actions. A belief strategy of player 1 is a strategy whose memory is $\mathcal{P}(K)$ and the update function coincides with $\mathcal{B}_{1}$ on $\mathcal{P}(K) \backslash\{\emptyset\}$. A 2 -belief strategy of player 1 is a strategy whose memory is a subset of $\mathcal{P}(K \times \mathcal{P}(K))$, and the update coincides with $\mathcal{B}_{1}^{(2)}$ on $\mathcal{P}(K \times \mathcal{P}(K)) \backslash\{\emptyset\}$.

Remark that in a belief strategy, by definition, the memory update is deterministic from every memory state different from $\emptyset$. However it may be randomised from $\emptyset$. Actually, in the positively winning 2-belief strategies of player 2 for Büchi games built in this paper (cf Theorem 6.6), $\emptyset$ is the initial memory state and, whatever signal is received, the update function sets positive chance to stay in $\emptyset$ as well as perform a transition to other memory states.

\subsection{Particular signalling structures}

To give a complete picture of stochastic games with signals, and to compare with existing work on games with imperfect information, we will at some places consider restricted classes of games, based on their signalling structures, as defined below. 
Definition 5.4. $\quad$ Player 1 is perfectly informed about the state if her signals reveal the state i.e. if for every signal $c \in C$ of player 1 there is a state $k_{c} \in K$ such that $p\left(k^{\prime}, c, d \mid k, i, j\right)>0 \Longrightarrow k^{\prime}=k_{c}$.

Player 1 is better informed than player 2 if her signals reveal the signals received by player 2 i.e. if for every signal $c \in C$ of player 1 there is a signal $d_{c} \in D$ of player 2 such that $p\left(k^{\prime}, c, d \mid k, i, j\right)>0 \Longrightarrow d=d_{c}$.

Player 1 is perfectly informed if she is both perfectly informed about the state and better informed than player 2 .

In the games of incomplete information used in Chatterjee et al. 2007, being perfectly informed is equivalent to being perfectly informed about the state, as the signal received by a player is entirely determined by the state of the game. However, in stochastic games with signals, a player may be perfectly informed about the state and yet not know the signal received by their opponent.

\section{MAIN RESULTS.}

In this section we state our main contributions, and the proofs can be found in the next sections.

\subsection{Qualitative Determinacy.}

The following theorem constitutes the core of the paper.

THEOREM 6.1. Stochastic games with signals and reachability, safety and Büchi winning conditions are qualitatively determined.

The proof can be found in Section 7.

Since reachability and safety games are dual, a consequence of Theorem 6.1, is that in a reachability game, every initial distribution is either almost-surely winning for player 1 , almost-surely winning for player 2 , or positively winning for both players. When a safety condition is satisfied almost-surely for a fixed profile of strategies, it trivially implies that the safety condition is satisfied by all consistent plays, thus for safety games winning surely is the same than winning almost-surely.

By contrast, Büchi games are not qualitatively determined, a counter-example is given in Section 7.2. For Theorem 6.1 to hold players should be allowed to use general strategies (or equivalently mixed strategies) and finite-memory strategy with randomized updates. Otherwise, if players are restricted to behavioural strategies or finite-memory with deterministic updates then qualitative determinacy does not hold anymore, as demonstrated by Example 2.9.

\subsection{Algorithmic complexity of deciding the winner.}

We now turn to the result concerning the (time) complexity to decide stochastic games with signals, starting with the easy case of safety games.

Proposition 6.2. In a safety game with signals, deciding whether the initial distribution is almost-surely winning for player 1 is EXPTIME-complete. If player 1 is perfectly informed about the state, the decision problem is in PTIME.

Almost-surely winning a safety game coincides with winning surely this safety game, which in turn coincides with winning surely against a perfectly informed opponent, thus Proposition 6.2 can be obtained by applying Reif 1979, Chatterjee et al. 2007] which tackle sure-winning in partially observable games.

Beside the determinacy result stated in Theorem 6.1 the main contribution of this article concerns the complexity of deciding reachability and Büchi games, for which we will establish the following theorem: 


\begin{tabular}{|l|c|c|}
\hline & Almost-surely & Positively \\
\hline Reachability & exponential & memoryless \\
\hline Safety & exponential & doubly-exponential \\
\hline Büchi & exponential & infinite \\
\hline Co-Büchi & infinite & doubly-exponential \\
\hline
\end{tabular}

Fig. 8. Tight memory requirements for finite-memory strategies with randomised updates.

THEOREM 6.3. In reachability and Büchi games with signals, deciding whether the initial distribution is almost-surely winning for player 1 is 2EXPTIME-complete.

Concerning winning positively a safety or co-Büchi game, one can use Theorem 6.1 and the determinacy property: player 2 has a positively winning strategy in the above game if and only if player 1 has no almost-surely winning strategy. Therefore, deciding when player 2 has a positively winning strategy can also be done, with the same complexity. The proof of the upper bound of Theorem 6.3 can be found in Section 8. The lower bound can be found in Theorem 10.1 .

For particular signalling structures, the complexity is better than 2EXPTIME, for example EXPTIME when player 2 is perfectly informed [Chatterjee et al. 2007]. This reduced complexity holds for other cases as well:

TheOREM 6.4. For reachability and Büchi games where either player 1 is perfectly informed about the state or player 2 is better informed than player 1, deciding whether the initial distribution is almost-surely winning for player 1 is EXPTIME-complete.

The upper bound in Theorem 6.4 is shown in Proposition 10.2. The winning states can be computed by the same fix-point algorithm used for Theorem 6.3 without any change. The lower bound derives from [Chatterjee et al. 2007.

\subsection{Complexity of strategies}

The doubly exponential time complexity of Theorem 6.3 is surprising. The main explanation to the time complexity is that a player may need doubly exponential memory to win positively. More generally, algorithmic complexity of these games is highly related to the memory needed by winning strategies, and finite-memory is sufficient to win every decidable game we consider in this paper. We give the precise tight memory requirements in Fig. 8 .

First, as already mentioned for Proposition 6.2, almost-surely winning safety games is equivalent with surely winning safety games. Hence, results of Reif 1979, Chatterjee et al. 2007] can be applied, giving the exponential upper-bound for the memory size needed for (almost-)surely winning safety games. More precisely, belief strategies are sufficient to win (almost-)surely safety games.

The upper-bound on memory for almost-surely winning reachability and Büchi games can be derived from the proof of the determinacy of reachability and Büchi games (see Corollary 8.1). Here again, belief-based strategies are sufficient to win almost-surely reachability and Büchi games. This is not very surprising since similar strategies were used in Chatterjee et al. 2007. where this result was used for games where player 2 has perfect information.

Proposition 6.5 (Belief strategies are sufficient to Win Almost-Surely). In safety, reachability and Büchi games with observable actions if a player wins almostsurely then the player has an almost-surely winning belief strategy. There are games for which strategies with an exponential number of memory states are necessary for a player to win almost-surely.

A very similar result holds in case the actions are not assumed to be observable. The only difference is that the finite-memory strategy is not exactly a belief strategy. Actually, 
there is a transformation of a belief strategy in $\operatorname{Obs}(\mathcal{A})$ to the corresponding equivalent finite-memory strategy in $\mathcal{A}$, as described by Lemma 4.8 . Inspecting the proof shows that the resulting strategy has memory $\mathcal{P}(K) \times I$ and its update operator coincides with $\mathcal{B}_{1}$ on the first component.

We now turn to the memory needed to win positively. First, memoryless strategies playing uniformly at random are sufficient to win positively reachability games.

A surprising fact is the amount of memory needed for winning positively co-Büchi and safety games. In these situations, it is still enough for a player to use a strategy with finitememory, but an exponential memory size is not enough to win positively. Actually, 2-belief strategies are sufficient for positively winning safety and co-Büchi games, and there is a doubly-exponential lower bound on memory for winning positively a safety or co-Büchi game (see Proposition 9.1p. This result cannot be derived from the memory requirements for player 1 to win almost-surely, nor from the work in Gripon and Serre 2009.

These bounds on the memory hold for finite-memory strategies with randomised updates. When only deterministic updates are considered and actions are not observable, memory requirements can become non-elementary (see Chatterjee and Doyen 2012] and the discussion in Section 2.2.

THEOREM 6.6 (2-BELIEF STRATEGIES ARE SUFFICIENT TO WIN POSITIVELY). In reachability and Büchi games with observable actions, if player 2 wins positively then he has a positively winning 2-belief strategy. There are reachability games with signals where player 1 is better informed than player 2 and where strategies with a doubly exponential number of memory states are necessary for player 2 to win positively.

Like for Proposition 6.5 a very similar result holds in case the actions are not assumed to be observable, except the finite-memory strategy is not exactly a 2-belief strategy but rather the result of the linear transformation of a 2-belief strategy described in Lemma 4.8 .

Last, the infinite lower bound for positively winning Büchi games is a consequence of Baier et al. 2008 and Chatterjee et al. 2010 and the infinite lower bound for almostsurely winning co-Büchi games follows, since the class of languages recognised by probabilistic Büchi automata Baier et al. 2008 is closed by complementation.

\section{QUALITATIVE DETERMINACY OF STOCHASTIC GAMES WITH SIGNALS.}

\subsection{Qualitative determinacy of reachability, Büchi and safety games.}

The goal of this subsection is to prove Theorem 6.1 that states the qualitative determinacy of reachability, Büchi and safety games. Note that the qualitative determinacy of Büchi games implies the qualitative determinacy of reachability games, since any reachability game can be turned into an equivalent Büchi one by making all target states absorbing. Qualitative determinacy of safety games is rather easy to establish, so we omit the proof here. Proving qualitative determinacy of Büchi games is harder and we provide full details.

7.1.1. Properties of beliefs. The following properties of beliefs are useful.

LEMMA 7.1. Let $\mathcal{A}$ be an arena with observable actions and $\tau_{\text {rand }}$ the strategy of player 2 which always plays the uniform distribution over J. For every behavioural strategies $\sigma$ and $\tau$, initial distribution $\delta$ and $n \in \mathbb{N}$, the following statements hold $\mathbb{P}_{\delta}^{\sigma, \tau}$-almost-surely:

$$
\begin{aligned}
& \mathcal{B}_{1}^{n}=\left\{k \in K \mid \mathbb{P}_{\delta}^{\sigma, \tau_{\text {rand }}}\left(K_{n}=k \mid C_{1}, \ldots, C_{n}\right)>0\right\} \\
& K_{n} \in \mathcal{B}_{1}^{n} .
\end{aligned}
$$

Remark that (6) is an equality between random variables: $\mathcal{B}_{1}^{n}=\mathcal{B}_{1}\left(\operatorname{supp}(\delta), C_{1} \cdots C_{n}\right)$ is $\left(C_{1}, \ldots, C_{n}\right)$-measurable. 
The proof of Lemma 7.1 relies on the following lemma, called the shifting lemma, which describes the effect of shifting time on the probability measure induced by two behavioural strategies.

Lemma 7.2 (Shifting LEMma). For every $n \in \mathbb{N}$, we denote $P_{\geq n}$ the infinite suffix of the play: $P_{\geq n}=K_{n}, I_{n}, J_{n}, C_{n+1}, D_{n+1}, K_{n+1}, \ldots$ Let $\delta$ be an initial distribution and $\sigma$ and $\tau$ two behavioural strategies. Let $c \in C$ and $d \in D$ and $\delta_{(c, d)}$ be the probability distribution on states and $\sigma_{c}$ and $\tau_{d}$ be the strategies defined by

$$
\begin{aligned}
& \delta_{c d}(k)=\mathbb{P}_{\delta}^{\sigma, \tau}\left(K_{1}=k \mid C_{1}=c, D_{1}=d\right) \\
& \sigma_{c}: c_{2} c_{3} \cdots c_{n} \mapsto \sigma\left(c c_{2} c_{3} \cdots c_{n}\right) \\
& \tau_{d}: d_{2} d_{3} \cdots d_{n} \mapsto \tau\left(d d_{2} d_{3} \cdots d_{n}\right) .
\end{aligned}
$$

Then for every measurable event $E \subseteq K(I J C D)^{\omega}$,

$$
\mathbb{P}_{\delta}^{\sigma, \tau}\left(P_{\geq 1} \in E \mid C_{1}=c, D_{1}=d\right)=\mathbb{P}_{\delta_{(c, d)}}^{\sigma_{c}, \tau_{d}}(E)
$$

More generally, for every $n \in \mathbb{N}$,

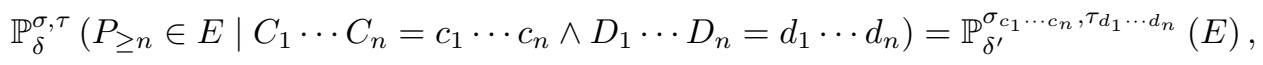

where $\sigma_{c_{1} \cdots c_{n}}(p)=\sigma\left(c_{1} \cdots c_{n} p\right)$ and $\tau_{c_{1} \cdots c_{n}}$ is defined similarly and $\delta^{\prime}(k)=$ $\mathbb{P}_{\delta}^{\sigma, \tau}\left(K_{n}=k \mid C_{1} \cdots C_{n}=c_{1} \cdots c_{n} \wedge D_{1} \cdots D_{n}=d_{1} \cdots d_{n}\right)$.

Proof. Using the definition of the probability measure $\mathbb{P}_{\delta}^{\sigma, \tau}, 8$ holds when $E$ is a finite union of cylinders. Moreover the class of events $E$ that satisfy property $(8)$ is clearly closed under countable monotone unions and intersection thus it is a monotone class. Thus, according to the monotone class theorem [Durrett 2010, Theorem 6.1.3, page 235] all measurable events have property (8). The proof of (9) follows by induction.

The proof of (8)

Proof of Lemma 7.1. First, (6) holds for $n=1$. Let $c \in C$ such that $\mathbb{P}_{\delta}^{\sigma, \tau}\left(C_{1}=c\right)>0$. Then $\sigma(\varepsilon)\left(\operatorname{Act}_{1}(c)\right)>0$ and

$$
\begin{aligned}
& \left\{k \in K \mid \mathbb{P}_{\delta}^{\sigma, \tau_{\text {rand }}}\left(K_{1}=k \mid C_{1}=c\right)>0\right\} \\
& =\left\{k \in K \mid \exists k^{\prime} \in K, \exists d \in D, \mathbb{P}_{\delta}^{\sigma, \tau_{\text {rand }}}\left(K_{1}=k^{\prime}, K_{0}=k, D_{1}=d \mid C_{1}=c\right)>0\right\} \\
& =\left\{k \in K \mid \exists k^{\prime} \in \operatorname{supp}(\delta), d \in D, p\left(k^{\prime}, C_{1}, d \mid k, \operatorname{Act}_{1}(c), \operatorname{Act}_{2}(d)\right)>0\right\} \\
& =\mathcal{B}_{1}(\operatorname{supp}(\delta), c) .
\end{aligned}
$$

where the first equality is by additivity, the second because all possible actions are played by $\tau_{\text {rand }}$ and $\sigma(\varepsilon)\left(\operatorname{Act}_{1}(c)\right)>0$, and the last by definition of the operator $\mathcal{B}_{1}$. Since $\operatorname{supp}(\delta)=$ $\mathcal{B}_{1}^{0}$ then (6) holds $\mathbb{P}_{\delta}^{\sigma, \tau}$-almost-surely for $n=1$. The case for arbitrary $n \in \mathbb{N}$ follows from an induction based on (8) of the shifting Lemma.

According to (6), equation (7) holds $\mathbb{P}_{\delta}^{\sigma, \tau_{\text {rand }}}$-almost-surely. Since $\tau_{\text {rand }}$ plays every possible action with positive probability, $\left(\mathbb{P}_{\delta}^{\sigma, \tau}\left(K_{n}=k\right)>0\right) \Longrightarrow\left(\mathbb{P}_{\delta}^{\sigma, \tau_{\text {rand }}}\left(K_{n}=k\right)>0\right)$, thus $(7)$ holds $\mathbb{P}_{\delta}^{\sigma, \tau}$-almost-surely.

We use the following technical lemma about belief-based strategies several times.

LEMma 7.3. Fix a Büchi game with observable actions. Let $\mathcal{L} \subseteq \mathcal{P}(K)$ and $\sigma$ a strategy for player 1 . Assume that $\sigma$ is a belief strategy, $\mathcal{L}$ is downward-closed, and for every $L \in \mathcal{L}$ and every strategy $\tau$,

$$
\begin{aligned}
& \mathbb{P}_{\delta_{L}}^{\sigma, \tau}\left(\exists n \in \mathbb{N}, K_{n} \in T\right)>0, \\
& \mathbb{P}_{\delta_{L}}^{\sigma, \tau}\left(\forall n \in \mathbb{N}, \mathcal{B}_{1}^{n} \in \mathcal{L}\right)=1 .
\end{aligned}
$$

Then $\sigma$ is almost-surely winning for the Büchi game from any non-empty support $L \in \mathcal{L}$. 
Proof. Since $\mathcal{L}$ is downward-closed then $\forall L \in \mathcal{L}, \forall l \in L,\{l\} \in \mathcal{L}$ thus 10 implies

$$
\forall L \in \mathcal{L}, \forall l \in L, \mathbb{P}_{\delta_{L}}^{\sigma, \tau}\left(\exists n \in \mathbb{N}, K_{n} \in T \mid K_{0}=l\right)>0
$$

Once $\sigma$ is fixed then the game is a one-player game with state space $K \times 2^{K}$ and imperfect information and $(12)$ implies

$$
\forall L \in \mathcal{L}, \forall l \in L, \forall \tau, \mathbb{P}_{\delta_{L}}^{\tau}\left(\exists n \leq N, K_{n} \in T \mid K_{0}=l\right)>\varepsilon,
$$

where $N=|K| \cdot \mid 2^{|K|}$ and $\varepsilon=p_{\min }^{|K| \cdot 2^{|K|}}$ and $p_{\min }$ is the minimal non-zero transition probability. Moreover (11) implies that in this one-player game the second component of the state space is always in $\mathcal{L}$, whatever strategy $\tau$ is played by player 2 . As a consequence, in this one-player game for every $m \in \mathbb{N}$, and every behavioural strategy $\tau$ and every $l \in K$,

$$
\mathbb{P}_{\delta_{L}}^{\tau}\left(\exists m \leq n \leq m+N, K_{n} \in T \mid K_{m}=l\right) \geq \varepsilon
$$

whenever $\mathbb{P}_{\delta_{L}}^{\tau}\left(K_{m}=l\right)>0$. We use the Borel-Cantelli Lemma to conclude the proof. According to (14), for every $\tau, L \in \overline{\mathcal{L}}, m \in \mathbb{N}$,

$$
\mathbb{P}_{\delta_{L}}^{\tau}\left(\exists n, m N \leq n<(m+1) N, K_{n} \in T \mid K_{m N}\right) \geq \varepsilon,
$$

which implies for every behavioural strategy $\tau$ and $k, m \in \mathbb{N}$,

$$
\mathbb{P}_{\delta_{L}}^{\tau}\left(\forall n,(m \cdot N) \leq n<((m+k) \cdot N) \Longrightarrow K_{n} \notin T\right) \leq(1-\varepsilon)^{k}
$$

Since $\sum_{k}(1-\varepsilon)^{k}$ is finite, we can apply Borel-Cantelli Lemma for the events $(\{\forall n, m \cdot N \leq$ $\left.\left.n<(m+k) \cdot N \Longrightarrow K_{n} \notin T\right\}\right)_{k}$ and we get $\mathbb{P}_{\delta_{L}}^{\tau}\left(\forall n, m \cdot N \leq n \Longrightarrow K_{n} \notin T\right)=0$ thus

$$
\mathbb{P}_{\delta_{L}}^{\tau}(\text { Büchi })=1 \text {. }
$$

As a consequence $\sigma$ is almost-surely winning for the Büchi game.

7.1.2. The maximal strategy. In every Büchi game with observable actions we define a beliefbased strategy $\sigma_{\max }$ called the maximal strategy of player 1 and we prove that this strategy is almost-surely winning from any initial distribution which is not positively winning for player 2. The maximal strategy is quite simple to define, as follows.

Definition 7.4 (Maximal strategy). Fix a Büchi game with observable actions. Let $\mathcal{L} \subseteq$ $\mathcal{P}(K) \backslash\{\emptyset\}$ be the set of supports that are positively winning for player 2 . For every $L \subseteq K$ we define the set of $L$-safe actions

$$
\operatorname{ISafe}_{\mathcal{L}}(L)=\left\{i \in I \mid \forall c \in C,\left(\operatorname{Act}_{1}(c)=i\right) \Longrightarrow\left(\mathcal{B}_{1}(L, c) \notin \mathcal{L}\right)\right\} .
$$

The maximal strategy is the belief strategy of player 1 which plays the uniform distribution on $\operatorname{ISafe}_{\mathcal{L}}\left(\mathcal{B}_{1}\right)$ when it is not empty and plays the uniform distribution on $I$ otherwise.

An important feature of the maximal strategy is the following.

Lemma 7.5. In a Büchi game with observable actions, let $\delta \in \Delta(K)$ be an initial distribution which is not positively winning for player 2 , i.e. $\operatorname{supp}(\delta) \notin \mathcal{L}$. Then for every strategy $\tau$ of player 2 and every $n \in \mathbb{N}$

$$
\mathbb{P}_{\delta}^{\sigma_{\max }, \tau}\left(\mathcal{B}_{1}^{n} \notin \mathcal{L}\right)=1
$$

Proof. The proof is by induction on $n$. The case $n=0$ is by hypothesis since $\mathbb{P}_{\delta}^{\sigma_{\text {max }}, \tau}\left(\mathcal{B}_{1}^{0}=\operatorname{supp}(\delta)\right)=1$. Let $\overline{\mathcal{L}}=\mathcal{P}(K) \backslash(\mathcal{L} \cup\{\emptyset\})$. To perform the inductive step, it is actually enough to prove

$$
\forall L \in \overline{\mathcal{L}}, \operatorname{ISafe}_{\mathcal{L}}(L) \neq \emptyset .
$$

Assume that 17 holds and that $\mathbb{P}_{\delta}^{\sigma_{\max }, \tau}\left(\mathcal{B}_{1}^{n} \in \overline{\mathcal{L}}\right)=1$. By definition of observability of actions, $\mathbb{P}_{\delta}^{\sigma_{\max }, \tau}\left(I_{n}=\operatorname{Act}_{1}\left(C_{n+1}\right)\right)=1$. Thus by definition of $\operatorname{ISafe}_{\mathcal{L}}(L)$, 
$\mathbb{P}_{\delta}^{\sigma_{\text {max }}, \tau}\left(\mathcal{B}_{1}\left(\mathcal{B}_{1}^{n}, C_{n+1}\right) \in \overline{\mathcal{L}} \mid I_{n} \in \operatorname{ISafe}_{\mathcal{L}}(L)\right)=1$. This concludes the inductive step since $\mathcal{B}_{1}\left(\mathcal{B}_{1}^{n}, C_{n+1}\right)=\mathcal{B}_{1}^{n+1}$ and $\mathbb{P}_{\delta}^{\sigma_{\max }, \tau}\left(I_{n} \in \operatorname{ISafe}_{\mathcal{L}}(L)\right)=1$.

The proof of $(17)$ is by contradiction. Assume that $\operatorname{ISafe}_{\mathcal{L}}(L)=\emptyset$ for some $L \in \overline{\mathcal{L}}$. Then for every action $i \in I$ there exists a signal $c_{i} \in C$ such that $\mathcal{B}_{1}\left(L, c_{i}\right) \neq \emptyset$ and $\mathcal{B}_{1}\left(L, c_{i}\right) \in \mathcal{L}$. Since $\mathcal{B}_{1}\left(L, c_{i}\right) \neq \emptyset$, the definition of the belief operator implies:

$$
\exists l_{i} \in L, k_{i} \in K, j_{i} \in J, d_{i} \in D \text {, such that } p\left(k_{i}, c_{i}, d_{i} \mid l_{i}, i, j_{i}\right)>0 .
$$

We prove:

$$
\forall \sigma, \mathbb{P}_{\delta_{L}}^{\sigma, \tau_{\text {rand }}}\left(\mathcal{B}_{1}^{1} \in \mathcal{L}\right)>0
$$

By definition of the probability measure $\mathbb{P}_{\delta_{L}}^{\sigma, \tau_{\text {rand }}}$ when $\sigma$ is a general strategy, it is enough to prove (18) when $\sigma$ is a behavioural strategy $\sigma: C^{*} \rightarrow \Delta(I)$. Let $I^{\prime}=\operatorname{supp}(\sigma(\varepsilon))$ and $i \in I^{\prime}$. Since $\tau_{\text {rand }}\left(j_{i}\right)>0$ there is non-zero probability that player 1 receives $c_{i}$ and then by choice of $c_{i}, \mathcal{B}_{1}\left(L, c_{i}\right) \in \mathcal{L}$. This proves (18).

To get the contradiction, we define a strategy $\tau^{\prime}$ for player 2 which is positively winning from $\delta_{L}$. By definition of $\mathcal{L}$ for every support $B \in \mathcal{L}$ there exists a positively winning strategy $\tau_{B}$ from the uniform initial distribution $\delta_{B}$. Since actions are observable then according to Theorem 4.9 we can assume w.l.o.g. that $\tau_{B}$ is behavioural. Let $\tau^{\prime}$ be the general strategy which plays the uniform distribution over $J$ for the first round, then at the beginning of the second round it selects at random some support $B \in \mathcal{L}$ and then plays $\tau_{B}$ from the second round until the end. According to (18), there exists $c \in C$ such that $\mathcal{B}_{1}(L, c) \in \mathcal{L}$ and

$$
\mathbb{P}_{\delta_{L}}^{\sigma, \tau^{\prime}}\left(C_{1}=c\right)>0
$$

We fix such a $c$ and we set $B=\mathcal{B}_{1}(L, c)$.

Although $\tau^{\prime}$ is not defined like a behavioural strategy, since actions are observable $\tau^{\prime}$ is equivalent to a behavioural strategy (Theorem 4.9). Thus we can apply the shifting lemma (Lemma 7.2) to $\delta_{L}, \sigma, \tau^{\prime}$ and CoBüchi and with the same notations we get:

$$
\forall d \in D, \mathbb{P}_{\delta_{L}}^{\sigma, \tau^{\prime}}\left(\text { CoBüchi } \mid C_{1}=c, D_{1}=d\right)=\mathbb{P}_{\delta_{c d}^{\prime}}^{\sigma_{c}, \tau_{d}^{\prime}} \text { (CoBüchi) },
$$

with $\delta_{c d}^{\prime}(k)=\mathbb{P}_{\delta_{L}}^{\sigma, \tau^{\prime}}\left(K_{1}=k \mid C_{1}=c, D_{1}=d\right)$. Since $\tau^{\prime}$ plays the same way independently of the first signal $D_{1}, \tau_{d}^{\prime}$ is independent of $d$ : actually $\tau_{d}^{\prime}$ is the strategy which selects randomly any $B \in \mathcal{L}$ and plays $\tau_{B}$ forever. Denote $\tau^{\prime \prime}$ this strategy. Summing 20 over all $d \in D$, weighted by $\mathbb{P}_{\delta_{L}}^{\sigma, \tau^{\prime}}\left(D_{1}=d\right)$ we get

$$
\mathbb{P}_{\delta_{L}}^{\sigma, \tau^{\prime}}\left(\text { CoBüchi } \mid C_{1}=c\right)=\mathbb{P}_{\delta_{c}^{\prime}}^{\sigma_{c}, \tau^{\prime \prime}}(\text { CoBüchi })
$$

with $\delta_{c}^{\prime}(k)=\mathbb{P}_{\delta_{L}}^{\sigma, \tau^{\prime}}\left(K_{1}=k \mid C_{1}=c\right)$. Let $B^{\prime}=\operatorname{supp}\left(\delta_{c}^{\prime}\right)$. According to the properties of beliefs (Lemma 7.1), since $\tau^{\prime}$ plays randomly for the first round, $B^{\prime}=\mathcal{B}_{1}(L, c)=B$. By definition of $\tau^{\prime \prime}$, there is positive chance that $\tau^{\prime \prime}$ plays like $\tau_{B}$ forever, and $\tau_{B}$ is positively winning from $B$ thus

$$
\mathbb{P}_{\delta_{c}^{\prime}}^{\sigma_{c}, \tau^{\prime \prime}}(\text { CoBüchi })>0
$$

According to 21) it implies $\mathbb{P}_{\delta_{L}}^{\sigma, \tau^{\prime}}\left(\right.$ CoBüchi $\left.\mid C_{1}=c\right)>0$ which together with 19 implies

$$
\mathbb{P}_{\delta_{L}}^{\sigma, \tau^{\prime}}(\mathrm{CoBüchi})>0
$$

Since this holds for every behavioural strategy $\sigma$, the strategy $\tau^{\prime}$ is positively winning from support $\delta_{L}$ thus $L \in \mathcal{L}$, a contradiction with $L \in \overline{\mathcal{L}}$. This completes the proof of (17).

The notion of maximal strategy being defined, we can complete the proof of Theorem 6.1. 
7.1.3. Proof of Theorem 6.1. Reachability and safety conditions can be easily encoded as Büchi conditions, thus it is enough to prove Theorem 6.1 for Büchi games. We prove Theorem 6.1 in the case where actions are observable, which implies that Theorem 6.1 holds in every arena, according to Theorem 4.9

In this case, the maximal strategy $\sigma_{\max }$ is well-defined.

Since $\mathcal{L}$ is the collection of positively winning supports for player 2 , it is enough to show that the maximal strategy is almost-surely winning from every support not in $\mathcal{L}$.

Let $\overline{\mathcal{L}}=\mathcal{P}(K) \backslash(\mathcal{L} \cup\{\emptyset\})$. The first step is to prove that for every $L \in \overline{\mathcal{L}}$,

$$
\forall k_{0} \in L, \forall \tau, \mathbb{P}_{\delta_{L}}^{\sigma_{\max }, \tau}(\text { Safe })<1
$$

We prove 22 by contradiction. Assume 22 does not hold for some $L \in \overline{\mathcal{L}}$ and strategy $\tau$ :

$$
\mathbb{P}_{\delta_{L}}^{\sigma_{\max }, \tau}(\text { Safe })=1
$$

Under this assumption we use $\tau$ to build a strategy positively winning from $L$, which will contradict the hypothesis $L \in \overline{\mathcal{L}}$. Of course $\tau$ itself is not necessarily a positively winning strategy from $L$, the only sure thing is that it is positively winning against $\sigma_{\text {max }}$. Instead we define a general strategy $T^{\prime} \in \Delta\left(C^{*} \rightarrow \Delta(I)\right)$ as follows. The strategy $T^{\prime}$ is any general strategy which gives positive probability to play $\tau$ as well as any strategy in the family of strategies $\left(\tau_{n, B}\right)_{n \in \mathbb{N}, B \in \mathcal{L}}$ defined as follows. For every $B \in \mathcal{L}$ we choose a strategy $\tau_{B}$ positively winning from $B$. Then $\tau_{n, B}$ is the strategy which plays the uniform distribution on $J$ for the first $n$ steps then forgets past signals and switches definitively to $\tau_{B}$.

A possible way to implement the general strategy $T^{\prime}$ is as follows. At the beginning of the play player 2 tosses a fair coin. If the result is head then he plays $\tau$. Otherwise he keeps tossing coins and plays randomly an action in $J$ as long as he gets head. Then the day he gets tail he pick ups randomly some $B \in \mathcal{L}$ and starts playing $\tau_{B}$.

Now that $T^{\prime}$ is defined, we prove it is positively winning from $L$. Let $E$ be the event "player 1 plays only actions that are safe with respect to her belief", i.e.

$$
E=\left\{\forall n \in \mathbb{N}, I_{n} \in \operatorname{ISafe}_{\mathcal{L}}\left(\mathcal{B}_{1}^{n}\right)\right\}
$$

Then for every behavioural strategy $\sigma$ :

- Either $\mathbb{P}_{\delta_{L}}^{\sigma, T^{\prime}}(E)=1$. In this case

$$
\mathbb{P}_{\delta_{L}}^{\sigma, T^{\prime}}(\text { Safe })>0
$$

because for every finite play $\pi=k_{0} i_{0} j_{0} c_{1} d_{1} k_{1} \cdots k_{n}$,

$$
\left(\mathbb{P}_{\delta_{L}}^{\sigma, \tau}(\pi)>0\right) \Longrightarrow\left(\mathbb{P}_{\delta_{L}}^{\sigma_{\max }, \tau}(\pi)>0\right) \Longrightarrow\left(\forall 0 \leq m \leq n, k_{m} \notin T\right),
$$

where the first implication holds because, by definition of $\sigma_{\max }$ and $E$, for every $c_{1} \cdots c_{n} \in$ $C^{*}, \operatorname{supp}\left(\sigma\left(c_{1} \cdots c_{n}\right)\right) \subseteq \operatorname{supp}\left(\sigma_{\max }\left(c_{1} \cdots c_{n}\right)\right)$ while the second implication is from $(23)$. This guarantees $\mathbb{P}_{\delta_{L}}^{\sigma, \tau}($ Safe $)=1$ thus we get $\mathbb{P}_{\delta_{L}}^{\sigma, T^{\prime}}($ Safe $) \geq T^{\prime}(\tau)>0$ by definition of $T^{\prime}$.

- Or $\mathbb{P}_{\delta_{L}}^{\sigma, T^{\prime}}(E)<1$. Then by definition of $E$ there exists $n \in \mathbb{N}$ such that $\mathbb{P}_{\delta_{L}}^{\sigma, T^{\prime}}\left(I_{n} \notin \operatorname{ISafe}_{\mathcal{L}}\left(\mathcal{B}_{1}^{n}\right)\right)>0$. By definition of $\operatorname{ISafe}_{\mathcal{L}}$ it implies $\mathbb{P}_{\delta_{L}}^{\sigma, T^{\prime}}\left(\mathcal{B}_{1}^{n+1} \in \mathcal{L}\right)>0$, thus there exists $B \in \mathcal{L}$ such that $\mathbb{P}_{\delta_{L}}^{\sigma, T^{\prime}}\left(\mathcal{B}_{1}^{n+1}=B\right)>0$. By definition of $T^{\prime}$ we get $\mathbb{P}_{\delta_{L}}^{\sigma, \tau_{n+1, B}}\left(\mathcal{B}_{1}^{n+1}=B\right)>0$, because whatever finite play $k_{0}, \ldots, k_{n+1}$ leads with positive probability to the event $\left\{\mathcal{B}_{1}^{n+1}=B\right\}$, the same finite play can occur with $\tau_{n+1, B}$ since $\tau_{n+1, B}$ plays every possible action for the $n+1$ first steps. Since $\tau_{n+1, B}$ coincides with $\tau_{\text {rand }}$ for the first $n+1$ steps then according to $6 \mathbb{P}_{\delta_{L}}^{\sigma, \tau_{n+1, B}}\left(\mathcal{B}_{1}^{n+1}=B\right)>0$ and $B \subseteq\left\{k \in K \mid \mathbb{P}_{\delta_{L}}^{\sigma, \tau_{n+1, B}}\left(K_{n+1}=k \mid \mathcal{B}_{1}^{n+1}=B\right)>0\right\}$. Using the shifting lemma (both $\sigma$ 
and $\tau_{n+1, B}$ are behavioural) and the definition of $\tau_{B}$ we get $\mathbb{P}_{\delta_{L}}^{\sigma, \tau_{n+1, B}}$ (CoBüchi) $>0$. As a consequence by definition of $T^{\prime}$ we get that $\mathbb{P}_{\delta_{L}}^{\sigma, T^{\prime}}(\mathrm{CoBüchi})>0$.

In both cases, for every $\sigma, \mathbb{P}_{\delta_{L}}^{\sigma, T^{\prime}}$ (CoBüchi) $>0$ thus $T^{\prime}$ is positively winning from $L$. This contradicts the hypothesis of $L \in \overline{\mathcal{L}}$. As a consequence we get $(22)$ by contradiction.

Using 22 , we apply Lemma 7.3 to the collection $\overline{\mathcal{L}}$ and the strategy $\sigma_{\text {can }}$. The collection $\overline{\mathcal{L}}$ is downward-closed because $\mathcal{L}$ is upward-closed: if a support is positively winning for player 2 then any greater support is positively winning as well, using the same positively winning strategy.

Thus $\sigma_{\text {can }}$ is almost-surely winning for the Büchi game from every support in $\overline{\mathcal{L}}$ i.e. every support which is not positively winning for player 2 . This terminates the proof that Büchi games are qualitatively determined.

\subsection{Nondeterminacy of co-Büchi games.}

In contrast with Büchi games, not all co-Büchi games are qualitatively determined: a counter-example is represented on Fig. 9. Similar examples can be used to prove that stochastic Büchi games with signals do not have a value Gimbert et al. 2016. In this game, player 1 observes everything, player 2 is blind (he only observes his own actions), and player 1 's objective is to visit only finitely many times the $\odot-$-state. The initial state is $\odot$.

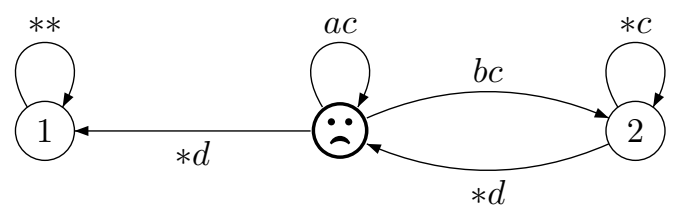

Fig. 9. Co-Büchi games are not qualitatively determined.

On one hand, no strategy $\Sigma$ is almost-surely winning for player 1 for the co-Büchi objective. According to Theorem 4.9, since both players can observe their actions, it is enough to prove that no behavioural strategy $\sigma \in C^{*} \rightarrow \Delta(I)$ of player 1 is almostsurely winning. Fix strategy $\sigma$ and assume towards contradiction that $\sigma$ is almost-surely winning. We define a strategy $\tau$ such that $\mathbb{P}_{\odot}^{\sigma, \tau}($ Büchi $)>0$. Strategy $\tau$ starts by playing only $c$. The probability to be in state $\odot$ at step $n$ is $x_{n}^{0}=\mathbb{P}_{\oplus}^{\sigma, c^{\omega}}\left(K_{n}=\odot\right)$ and since $\sigma$ is almost-surely winning then $x_{n}^{0} \rightarrow_{n} 0$ thus there exists $n_{0}$ such that $x_{n_{0}}^{0} \leq \frac{1}{2}$. Then $\tau$ plays $d$ at step $n_{0}$. Assuming the state was 2 when $d$ was played, the probability to be in state $\odot$ at step $n \geq n_{0}$ is $x_{n}^{1}=\mathbb{P}_{\odot}^{\sigma, c^{n}} d c^{\omega}\left(K_{n}=\odot \mid K_{n_{0}}=\odot\right)$ and since $\sigma$ is almost-surely winning there exists $n_{1}$ such that $x_{n_{1}}^{1} \leq \frac{1}{4}$. Then $\tau$ plays $d$ at step $n_{1}$. By induction we keep defining $\tau$ this way so that $\tau=c^{n_{0}-1} d c^{n_{1}-n_{0}-1} d c^{n_{2}-n_{1}-1} d \cdots$. and for every $k \in \mathbb{N}, \mathbb{P}_{\circledast}^{\sigma, \tau}\left(K_{n_{k+1}}=\odot\right.$ and $\left.K_{n_{k+1}-1}=2 \mid K_{n_{k}}=\odot\right) \geq 1-\frac{1}{2^{k+1}}$. Thus finally $\mathbb{P}_{\odot}^{\sigma, \tau}(\mathrm{Büchi}) \geq \Pi_{k}\left(1-\frac{1}{2^{k+1}}\right)>0$ which contradicts the hypothesis.

On the other hand, player 2 does not have a positively winning strategy either. Intuitively, player 2 cannot win positively because as time passes, either the play reaches state 1 or the chances that player 2 plays action $d$ drop to 0 . When these chances are small, player 1 can play action $c$ and she bets no more $d$ will be played and the play will stay safe in state 2 . If player 1 loses her bet then again she waits until the chances to see another $d$ are small and then plays action $c$. Player 1 may lose a couple of bets but almost-surely she eventually is right and the CoBüchi condition is fulfilled. Formally, according to Theorem 4.9, since 
both players can observe their actions, it is enough to prove that no behavioural strategy $\tau \in D^{*} \rightarrow \Delta(J)$ of player 2 is positively winning. The strategy $\tau$ being fixed, we define a strategy $\sigma$ for player 1 such that $\mathbb{P}_{\odot}^{\sigma, \tau}($ Büchi $)=1$. The only state where player 1's action matters is $\odot$. After a play $p=k_{0} i_{0} j_{0} \cdots k_{n}$ ending up in state $\odot$ (player 1 can observe the state), the strategy $\sigma$ plays action $a$ except if the trigger condition

$$
\mathbb{P}_{\circledast}^{i_{0} \cdots i_{n} a^{\omega}, \tau}\left(\forall m \geq n, J_{m} \neq d \mid P_{n}=p\right) \geq \frac{1}{2},
$$

is satisfied in this case action $b$ is played. Let $E_{0}$ the event that finitely many $d$ are played i.e.

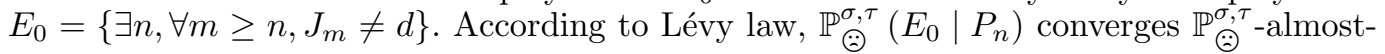
surely to the indicator function $1_{E_{0}}$ of the event $E_{0}$. If $E_{0}$ holds then finitely many $d$ are played, and the play cannot stay forever in state $\odot$ after the last $d$ because $\mathbb{P}_{\odot}^{\sigma, \tau}\left(E_{0} \mid P_{n}\right)$ converges to 1 thus the trigger condition is eventually satisfied. Thus when $E_{0}$ holds the play eventually stays in state 1 or 2 and the CoBüchi condition is satisfied. If $E_{0}$ does not hold then $\mathbb{P}_{\odot}^{\sigma, \tau}\left(E_{0} \mid P_{n}\right)$ converges to 0 thus eventually the trigger condition is not satisfied anymore hence player 1 eventually plays no more $b$ 's, only $a$ 's. But $E_{0}$ does not hold thus infinitely many $d$ are played, thus the play reaches state 1 . In both cases CoBüchi holds $\mathbb{P}_{\odot}^{\sigma, \tau}$-almost-surely, thus $\tau$ is not positively winning.

Finally neither player 1 wins almost-surely nor player 2 wins positively.

\section{ALGORITHMS}

\subsection{A naïve algorithm}

As a corollary of the proof of qualitative determinacy (Theorem 6.1), we get a maximal strategy $\sigma_{\max }$ for player 1 (see Definition 7.4 to win almost-surely Büchi games.

COROLlary 8.1. If player 1 has an almost-surely winning strategy in a Büchi game with observable actions then the maximal strategy $\sigma_{\max }$ is almost-surely winning.

A simple algorithm to decide for which player a game is winning can be derived from Corollary 8.1 this simple algorithm enumerates all possible belief strategies and test each one of them to see if it is almost-surely winning. The test reduces to checking positive winning in one-player co-Büchi games and can be done in exponential time. As there is a doubly exponential number of belief strategies, this can be done in time doubly exponential. This algorithm also appears in Gripon and Serre 2009. This settles the upper bound for Theorem 6.3. The lower bounds are established in Theorem 10.1, proving that this enumeration algorithm is optimal for worst case complexity. While optimal in the worst case, this algorithm is likely to be unefficient in practice. For instance, if player 1 has no almost-surely winning strategy, then this algorithm will enumerate every single of the doubly exponential many possible belief strategies. Instead, we provide fix-point algorithms which do not enumerate every possible strategy in Theorem 8.2 for reachability games and Theorem 8.3 for Büchi games. Although they should perform better on games with particular structures, these fix-point algorithms still have a worst-case 2-EXPTIME complexity.

\subsection{A fix-point algorithm for reachability games}

We turn now to the (fix-points) algorithms which compute the set of supports that are almost-surely or positively winning for various objectives.

TheOREM 8.2 (DECIDING POSITIVE WINNING IN REACHABILITy GAMES). In a reachability game each initial distribution $\delta$ is either positively winning for player 1 or surely winning for player 2 , and this depends only on $\operatorname{supp}(\delta) \subseteq K$. The corresponding partition of $\mathcal{P}(K)$ is computable in time $\mathcal{O}\left(|G| \cdot 2^{|K|}\right)$, where $|G|$ denotes the size of the description 
of the game, as the largest fix-point of a monotonic operator $\Phi: \mathcal{P}(\mathcal{P}(K)) \rightarrow \mathcal{P}(\mathcal{P}(K))$ computable in time linear in $|G|$.

Proof. Let $\mathcal{L}_{\infty} \subseteq \mathcal{P}(K \backslash T)$ be the greatest fix-point of the monotonic operator $\Phi$ : $\mathcal{P}(\mathcal{P}(K \backslash T)) \rightarrow \mathcal{P}(\mathcal{P}(K \backslash T))$ defined by:

$$
\left.\Phi(\mathcal{L})=\left\{L \in \mathcal{L} \mid \exists j_{L} \in J, \forall d \in D, \operatorname{Act}_{2}(d)=j_{L}\right) \Longrightarrow\left(\mathcal{B}_{2}(L, d) \in \mathcal{L} \cup\{\emptyset)\right\}\right\},
$$

in other words $\Phi(\mathcal{L})$ is the set of supports such that player 2 has an action which ensure his next belief will be in $\mathcal{L}$, whatever signal $d$ he might receive. Let $\sigma_{\text {rand }}$ be the strategy for player 1 that plays randomly any action.

We are going to prove that:

(A) every support in $\mathcal{L}_{\infty}$ is surely winning for player 2 ,

(B) and $\sigma_{\text {rand }}$ is positively winning from any support $L \subseteq K$ which is not in $\mathcal{L}_{\infty}$.

We start with proving (A). To win surely from any support $L \in \mathcal{L}_{\infty}$, player 2 uses the following belief strategy $\tau_{B}$ : when the current belief of player 2 is $L \in \mathcal{L}_{\infty}$ then player 2 plays an action $j_{L}$ defined as in (24). By definition of $\Phi$ and since $\mathcal{L}_{\infty}$ is a fix-point of $\Phi$, there always exists such an action. When playing with the belief strategy $\tau_{B}$, starting from a support in $\mathcal{L}_{\infty}$, the beliefs of player 2 stay in $\mathcal{L}_{\infty}$ and never intersect $T$ because $\mathcal{L}_{\infty} \subseteq \mathcal{P}(K \backslash T)$. According to property $(7)$ of beliefs (Lemma 7.1), this guarantees the play never visits $T$, whatever strategy is used by player 1 .

We now prove $(\mathrm{B})$. Let $\mathcal{L}_{0}=\mathcal{P}(K \backslash T) \supseteq \mathcal{L}_{1}=\Phi\left(\mathcal{L}_{0}\right) \supseteq \mathcal{L}_{2}=\Phi\left(\mathcal{L}_{1}\right) \ldots$ and $\mathcal{L}_{\infty}$ be the limit of this sequence, the greatest fix-point of $\Phi$. We prove that for any support $L \in \mathcal{P}(K)$, if $L \notin \mathcal{L}_{\infty}$ then:

$$
\sigma_{\text {rand }} \text { is positively winning for player } 1 \text { from } L \text {. }
$$

If $L \cap T \neq \emptyset, 25$ is obvious. To deal with the case where $L \cap T=\emptyset$, we define for every $n \in \mathbb{N}, \mathcal{K}_{n}=\mathcal{P}(K \backslash T) \backslash \mathcal{L}_{n}$, and we prove by induction on $n \in \mathbb{N}$ that for every $L \in \mathcal{K}_{n}$, for every initial distribution $\delta_{L}$ with support $L$, for every behavioural strategy $\tau$,

$$
\mathbb{P}_{\delta_{L}}^{\sigma_{\text {rand }}, \tau}\left(\exists m, 2 \leq m \leq n+1, K_{m} \in T\right)>0 .
$$

For $n=0,26$ is obvious because $\mathcal{K}_{0}=\emptyset$. Suppose that for some $n \in \mathbb{N}$, (26) holds for every $L^{\prime} \in \mathcal{K}_{n}$, and let $L \in \mathcal{K}_{n+1} \backslash \mathcal{K}_{n}$. Then by definition of $\mathcal{K}_{n+1}$,

$$
L \in \mathcal{L}_{n} \backslash \Phi\left(\mathcal{L}_{n}\right) .
$$

Let $\delta_{L}$ be an initial distribution with support $L$ and $\tau$ any behavioural strategy for player 2 . Let $J_{0} \subseteq J$ be the support of $\tau\left(\delta_{L}\right)$ and $j_{L} \in J_{0}$. According to (27), by definition of $\Phi$, there exists a signal $d \in D$ such that $\operatorname{Act}_{2}(d)=j_{L}$ and $\mathcal{B}_{2}(L, d) \notin \mathcal{L}_{n}$ and $\mathcal{B}_{2}(L, d) \neq \emptyset$. According to property (6) of beliefs (Lemma 7.1), $\forall k \in \mathcal{B}_{2}(L, d), \mathbb{P}_{\delta_{L}}^{\sigma_{\text {rand }}, \tau}\left(K_{2}=k \wedge D_{1}=d\right)>0$. If $\mathcal{B}_{2}(L, d) \cap T \neq \emptyset$ then according to the definition of beliefs, $\mathbb{P}_{\delta_{L}}^{\sigma_{\text {rand }}, \tau}\left(K_{2} \in T\right)>0$. Otherwise $\mathcal{B}_{2}(L, d) \in \mathcal{P}(K \backslash T) \backslash \mathcal{L}_{n}=\mathcal{K}_{n}$ hence distribution $\delta_{d}: k \rightarrow \mathbb{P}_{\delta_{L}}^{\sigma_{\text {rand }}, \tau}\left(K_{2}=k \mid D_{1}=d\right)$ has its support in $\mathcal{K}_{n}$. By inductive hypothesis, for every behavioural strategy $\tau^{\prime}$,

$$
\mathbb{P}_{\delta_{d}}^{\sigma_{\text {rand }}, \tau^{\prime}}\left(\exists m \in \mathbb{N}, 2 \leq m \leq n+1, K_{m} \in T\right)>0
$$

hence using the shifting lemma and the definition of $\delta_{d}$,

$$
\mathbb{P}_{\delta}^{\sigma_{\text {rand }}, \tau}\left(\exists m \in \mathbb{N}, 3 \leq m \leq n+2, K_{m} \in T\right)>0,
$$

which completes the proof of the inductive step. Hence (26) holds for every behavioural strategy $\tau$. By definition of the probability measure associated with a general strategy, 26) holds as well for every general strategy $\tau$.

To compute the partition of supports between those positively winning for player 1 and those surely winning for player 2 , it is enough to compute the largest fix-point of $\Phi$. Since 
$\Phi$ is monotonic, and each application of the operator can be computed in time linear in the size of the game $(G)$ and the number of supports $\left(2^{|K|}\right)$ the overall computation can be achieved in time $|G| \cdot 2^{|K|}$. To compute the strategy $\tau_{B}$, it is enough to compute for each $L \in \mathcal{L}_{\infty}$ one action $j_{L}$ such that $\left(\operatorname{Act}_{2}(d)=j_{L}\right) \Longrightarrow\left(\mathcal{B}_{2}(L, d) \in \mathcal{L}_{\infty}\right)$.

As a byproduct of the proof one obtains the following bounds on time and probabilities before reaching a target state, when player 1 uses the uniform memoryless strategy $\sigma_{\text {rand }}$. From an initial distribution positively winning for the reachability objective, for every strategy $\tau$,

$$
\mathbb{P}_{\delta}^{\sigma_{\text {rand }}, \tau}\left(\exists n \leq 2^{|K|}, K_{n} \in T\right) \geq\left(\frac{1}{p_{\min }|I|}\right)^{2^{|K|}}
$$

where $p_{\min }$ is the smallest non-zero transition probability.

\subsection{A fix-point algorithm for Büchi games}

To decide whether player 1 wins almost-surely a Büchi game, we provide an algorithm which runs in doubly-exponential time. It uses the algorithm for reachability games as a sub-procedure.

TheOrem 8.3 (DECIDING ALMOST-SURE WINNING IN BüCHI GAMES). In a Büchi game each initial distribution $\delta$ is either almost-surely winning for player 1 or positively winning for player 2 , and this depends only on $\operatorname{supp}(\delta) \subseteq K$. The corresponding partition of $\mathcal{P}(K)$ is computable in time $\mathcal{O}\left(2^{2^{|G|}}\right)$, where $|G|$ denotes the size of the description of the game, as a projection of the greatest fix-point $\mathcal{L}_{\infty}$ of a monotonic operator

$$
\Psi: \mathcal{P}(\mathcal{P}(K) \times K) \rightarrow \mathcal{P}(\mathcal{P}(K) \times K)
$$

The operator $\Psi$ is computable using as a nested fix-point the operator $\Phi$ of Theorem 8.2. The almost-surely winning belief strategy of player 1 and the positively winning 2-belief strategy of player 2 can be extracted from $\mathcal{L}_{\infty}$.

The proof of Theorem 8.3 is detailed in subsection 8.4. We sketch here the main ideas.

First, suppose that from every initial support, player 1 can win positively the reachability game. Then she can do so using a belief strategy and according to 7.3 , this strategy guarantees almost-surely the Büchi condition.

In general though player 1 is not in such an easy situation and there exists a support $L$ which is not positively winning for her for the reachability objective. Then by qualitative determinacy, player 2 has a strategy to achieve surely her safety objective from $L$, which is a fortiori surely winning for her co-Büchi objective as well.

We prove that in case player 2 can force with positive probability the belief of player 1 to be $L$ eventually from another support $L^{\prime}$, then player 2 has a general strategy to win positively from $L^{\prime}$. This is not completely obvious because in general player 2 cannot know exactly when the belief of player 1 is $L$ (she can only compute the 2-Belief, letting him know all the possible beliefs player 1 can have). For winning positively from $L^{\prime}$, player 2 plays totally randomly until he guesses randomly that the belief of player 1 is $L$, at that moment he switches to a strategy surely winning from $L$. Such a strategy is far from being optimal, because player 2 plays randomly and in most cases he makes a wrong guess about the belief of player 1 . However player 2 wins positively because there is a non zero probability that he guesses correctly at the right moment the belief of player 1 .

Hence, player 1 should surely avoid her belief to be $L$ or $L^{\prime}$ if she wants to win almostsurely. However, doing so player 1 may prevent the play from reaching target states, which may create another positively winning support for player 2, and so on. This is the basis of our fix-point algorithm. 
Using these ideas, we prove that the set $\mathcal{L}_{\infty} \subseteq \mathcal{P}(K)$ of supports almost-surely winning for player 1 for the Büchi objective is the largest set of initial supports from which:

player 1 has a strategy which win positively the reachability game and also ensures at the same time her belief to stay in $\mathcal{L}_{\infty}$.

Property (†) can be reformulated as a reachability condition in a new game whose states are states of the original game augmented with beliefs of player 1, kept hidden to player 2 .

The fix-point characterisation suggests the following algorithm for computing the set of supports positively winning for player 2: $\mathcal{P}(K) \backslash \mathcal{L}_{\infty}$ is the limit of the sequence $\emptyset=\mathcal{L}_{0}^{\prime} \subsetneq$ $\mathcal{L}_{0}^{\prime} \cup \mathcal{L}_{1}^{\prime \prime} \subsetneq \mathcal{L}_{0}^{\prime} \cup \mathcal{L}_{1}^{\prime} \subsetneq \mathcal{L}_{0}^{\prime} \cup \mathcal{L}_{1}^{\prime} \cup \mathcal{L}_{2}^{\prime \prime} \subsetneq \ldots \subsetneq \mathcal{L}_{0}^{\prime} \cup \cdots \cup \mathcal{L}_{m}^{\prime}=\mathcal{P}(K) \backslash \mathcal{L}_{\infty}$, where

(a) from supports in $\mathcal{L}_{i+1}^{\prime \prime}$ player 2 can surely guarantee the safety objective, under the hypothesis that player 1 guarantees for sure her beliefs to stay outside $\mathcal{L}_{i}^{\prime}$,

(b) from supports in $\mathcal{L}_{i+1}^{\prime}$ player 2 can ensure with positive probability the belief of player 1 to be in $\mathcal{L}_{i+1}^{\prime \prime}$ eventually, under the same hypothesis.

The overall strategy of player 2 positively winning for the co-Büchi objective consists in playing randomly for some time until he decides to pick up randomly a belief $L$ of player 1 in some $\mathcal{L}_{i}^{\prime \prime}$ and bets that the current belief of player 1 is $L$ and that player 1 guarantees for sure her future beliefs will stay outside $\mathcal{L}_{i}^{\prime}$. . He forgets the signals he has received up to that moment and switches definitively to a strategy which guarantees (a). With positive probability, player 2 guesses correctly the belief of player 1 at the right moment, and future beliefs of player 1 will stay in $\mathcal{L}_{i}^{\prime}$, in which case the co-Büchi condition holds and player 2 wins.

In order to ensure (a), player 2 makes use of the hypothesis about player 1 beliefs staying outside $\mathcal{L}_{i}^{\prime}$. For that player 2 needs to keep track of all the possible beliefs of player 1 , hence the doubly-exponential memory. The reason is player 2 can infer from this data structure some information about the possible actions played by player 1: in case for every possible belief of player 1 an action $i \in I$ creates a risk to reach $\mathcal{L}_{i}^{\prime}$ then player 2 knows for sure this action is not played by player 1 . This in turn helps player 2 to know which are the possible states of the game. Finally, when player 2 estimates the state of the game using his 2-beliefs, this gives a potentially more accurate estimation of the possible states than simply computing his 1-beliefs.

The positively winning 2-belief strategy of player 2 has a particular structure. All memory updates are deterministic except for one: from the initial memory state $\emptyset$, whatever signal is received there is non-zero chance that the memory state stays $\emptyset$ but it may as well be updated to many other memory states.

\subsection{Proof of Theorem 8.3}

To establish Theorem 8.3 we start with formalising what it means for player 1 to enforce her beliefs to stay outside a certain set.

Definition 8.4. Let $\mathcal{L} \subseteq \mathcal{P}(K)$ be a set of non-empty supports. We say that player 1 can enforce her beliefs to stay outside $\mathcal{L}$ if player 1 has a strategy $\sigma$ such that for every strategy $\tau$ of player 2 and every initial distribution $\delta$ whose support is not in $\mathcal{L}$,

$$
\mathbb{P}_{\delta}^{\sigma, \tau}\left(\forall n \in \mathbb{N}, \mathcal{B}_{1}^{n} \notin \mathcal{L}\right)=1
$$

Equivalently, for every $L \notin \mathcal{L}$, the set:

$$
\operatorname{ISafe}_{\mathcal{L}}(L)=\left\{i \in I \mid \forall c \in C,\left(\operatorname{Act}_{1}(c)=i\right) \Longrightarrow\left(\mathcal{B}_{1}(L, c) \notin \mathcal{L}\right)\right\},
$$

of actions which guarantee the next belief of player 1 to stay outside $\mathcal{L}$ is not empty.

Note that the same operator $\operatorname{ISafe}_{\mathcal{L}}$ is also used in the proof of qualitative determinacy. 
Proof. The equivalence is straightforward. In one direction, let $\sigma$ be a strategy with the property (29), $L \notin \mathcal{L}, \delta_{L}$ a distribution with support $L$. Then according to (29), $\operatorname{supp}\left(\sigma\left(\delta_{L}\right)(i)\right) \subseteq \operatorname{ISafe}_{\mathcal{L}}(L)$ hence $\operatorname{ISafe}_{\mathcal{L}}(L)$ is not empty. In the other direction, if $\operatorname{ISafe}_{\mathcal{L}}(L)$ is not empty for every $L \notin \mathcal{L}$ then consider the finite-memory strategy $\sigma$ for player 1 which plays an action in $\operatorname{ISafe}_{\mathcal{L}}(L)$ when the belief of player 1 is $L$. Then by definition of $\operatorname{ISafe}_{\mathcal{L}}(L)$, and according to Lemma 7.1, property 29 holds.

We need also the notion of $\mathcal{L}$-games.

Definition 8.5 ( $\mathcal{L}$-games $)$. Let $\mathcal{L}$ be an upward-closed set of supports such that player 1 can enforce her beliefs to stay outside $\mathcal{L}$. The $\mathcal{L}$-game has same actions, transitions and signals than the original partial observation game, only the winning condition changes: player 1 wins if the play reaches a target state and moreover player 1 is restricted to use actions in $\operatorname{ISafe}_{\mathcal{L}}(L)$ whenever her belief is $L$. The winning condition is:

$$
\operatorname{Win}_{\mathcal{L}}=\left\{\exists n, K_{n} \in T \text { and } \forall n, I_{n} \in \operatorname{ISafe}_{\mathcal{L}}\left(\mathcal{B}_{1}^{n}\right)\right\} .
$$

Note that strictly speaking, $\mathcal{L}$-games are not reachability games however Theorem 8.2 also holds for these games.

The following properties of $\mathcal{L}$-games are crucial.

Proposition 8.6 ( $\mathcal{L}$-GAmes). Let $G$ be a Büchi game with observable actions. Let $\mathcal{L} \subseteq$ $\mathcal{P}(K)$ be a set of non-empty supports such that $\mathcal{L}$ is upward-closed and such that player 1 can enforce her beliefs to stay outside $\mathcal{L}$.

(i) In the $\mathcal{L}$-game, every support is either positively winning for player 1 or surely winning for player 2. We denote $\mathcal{L}^{\prime \prime}$ the set of supports that are not in $\mathcal{L}$ and are surely winning for player 2 in the $\mathcal{L}$-game.

(ii) Assume $\mathcal{L}^{\prime \prime}$ is empty. Then every support not in $\mathcal{L}$ is almost-surely winning for player 1 , both in the $\mathcal{L}$-game and also for the Büchi objective in game $G$.

(iii) Assume $\mathcal{L}^{\prime \prime}$ is not empty. Then player 2 has a 2-belief strategy $\tau$ with memory $\mathcal{P}\left(\mathcal{L}^{\prime \prime} \times\right.$ $K) \backslash\{\emptyset\}$ to win surely the $\mathcal{L}$-game from any support in $\mathcal{L}^{\prime \prime}$.

(iv) There is an algorithm running in time doubly-exponential in the size of $G$ to compute $\mathcal{L}^{\prime \prime}$ and, in case (iii) holds, strategy $\tau$. This algorithm performs the fix-point computation of Theorem 8.2 on a game with state space $\mathcal{P}((\mathcal{P}(K) \backslash \mathcal{L}) \times K)$.

Proof. We define a reachability game $G_{\mathcal{L}}$ which is similar to the $\mathcal{L}$-game. The game $G_{\mathcal{L}}$ is a synchronised product of the original game $G$ with beliefs of player 1, with a few modifications. The state space is $K_{\mathcal{L}}=K \times(\mathcal{P}(K) \backslash \mathcal{L} \cup\{\emptyset\})$. The first component is the state $K_{n}$ of the original game $G_{\mathcal{L}}$ and performs transitions according to the transition rules of the original game $G$. The second component keeps track of the belief of player 1, and in case player 1 plays a forbidden action $i \notin \operatorname{ISafe}_{\mathcal{L}}(B)$, this component is emptied definitively. Target states $T_{\mathcal{L}}$ of $G_{\mathcal{L}}$ are $T_{\mathcal{L}}=\{(s, B) \mid s \in T \wedge B \neq \emptyset\}$ so to win the game, a target state of the game $G$ should be entered while the belief has never been emptied.

Formally the non-zero values of the transition function $p_{\mathcal{L}}$ of $G_{\mathcal{L}}$ are defined for every $i \in I, j \in J$ and $k, k^{\prime} \in K$ and $B, B^{\prime} \subseteq K$ by $p_{\mathcal{L}}\left(\left(k^{\prime}, B^{\prime}\right), c, d \mid(k, B) i, j\right)=p\left(k^{\prime}, c, d \mid k, i, j\right)$ where, if $i \in \operatorname{ISafe}_{\mathcal{L}}(B)$,

$$
B^{\prime}= \begin{cases}\mathcal{B}_{1}(B, c) & \text { if }(B \neq \emptyset) \wedge\left(i \in \operatorname{ISafe}_{\mathcal{L}}(B)\right) \\ \emptyset & \text { otherwise }\end{cases}
$$

To get (i), (iii) and (iv) we apply Theorem 8.2 to the reachability game $G_{\mathcal{L}}$ : for every $L$, let $\delta_{\mathcal{L}}(L)$ the uniform distribution on $L \times\{L\}$, then $\delta_{\mathcal{L}}(L)$ is either positively winning for 1 or surely winning for 2 in $G_{\mathcal{L}}$. We show that the same holds for $L$ in the $\mathcal{L}$-game. 
Assume $\delta_{\mathcal{L}}(L)$ is positively winning for 1 in $G_{\mathcal{L}}$ then according to Theorem 8.2 the strategy $\sigma_{\text {rand }}$ which plays randomly all actions is positively winning in $G_{\mathcal{L}}$. By construction of $G_{\mathcal{L}}$, after signals $c_{1} \cdots c_{n}$ playing an action $i \notin \operatorname{ISafe}_{\mathcal{L}}\left(\mathcal{B}_{1}\left(c_{1} \cdots c_{n}\right)\right)$ is useless for player 1 since it empties the second component thus the probability to reach $T_{\mathcal{L}}$ is 0 onwards. Thus the strategy $\sigma_{\mathcal{L}}$ which plays randomly any action in $\operatorname{ISafe}_{\mathcal{L}}\left(\mathcal{B}_{1}\left(c_{1} \cdots c_{n}\right)\right)$ after signals $c_{1} \cdots c_{n}$ is positively winning as well in $G_{\mathcal{L}}$. Moreover, $\sigma_{\mathcal{L}}$ guarantees in $G$ that $\forall \tau, \mathbb{P}_{\delta}^{\sigma, \tau}\left(\forall n, \sigma\left(C_{1}, \ldots, C_{n}\right) \in \operatorname{ISafe}_{\mathcal{L}}\left(\mathcal{B}_{1}^{n}\right)\right)=1$ thus it is positively winning in the $\mathcal{L}$-game.

Assume now that $\delta_{\mathcal{L}}(L)$ is surely winning for player 2 in $G_{\mathcal{L}}$, then according to Theorem 8.2 player 2 can win surely with a belief strategy $\tau$. A belief strategy in $G_{\mathcal{L}}$ is a 2-belief strategy in $G$. According to the definition of $T_{\mathcal{L}}, \tau$ guarantees for sure in $G_{\mathcal{L}}$ that $\forall n \in \mathbb{N},\left(K_{n} \in T \times \mathcal{P}(K)\right) \Longrightarrow\left(K_{n} \in T \times\{\emptyset\}\right)$. Since $\tau$ is a strategy in the $\mathcal{L}$-game as well and by definition of transitions in $G_{\mathcal{L}}, \tau$ guarantees in the $\mathcal{L}$-game that $\forall n \in \mathbb{N},\left(K_{n} \in T\right) \Longrightarrow\left(\exists m \leq n, I_{m} \notin \operatorname{ISafe}_{\mathcal{L}}\left(\mathcal{B}_{1}^{n}\right)\right)$, thus $\tau$ is surely winning for player 2 in the $\mathcal{L}$-game.

This terminates the proof of (i), (iii) and (iv).

Now we suppose $\mathcal{L}^{\prime \prime}$ is empty and prove (ii). We use again the positively winning belief strategy $\sigma_{\mathcal{L}}$ defined above. We apply Lemma 7.3 to $\sigma_{\mathcal{L}}$ and $\overline{\mathcal{L}}=\mathcal{P}(K) \backslash \mathcal{L}$, which is downward-closed because $\mathcal{L}$ is upward-closed. For that we shall prove that the two hypotheses (10) and (11) are satisfied. Hypothesis (11) holds because $\sigma_{\mathcal{L}}$ only plays action in $I_{n} \in \operatorname{ISafe}_{\mathcal{L}}\left(\mathcal{B}_{1}^{n}\right)$ thus if the initial support is in $\overline{\mathcal{L}}$ then $\sigma_{\mathcal{L}}$ guarantees for sure $\forall n, \mathcal{B}_{1}^{n} \in \overline{\mathcal{L}}$. To prove 10 we need to show that for every $L \in \overline{\mathcal{L}}$ and $l \in L$, $\mathbb{P}_{\delta_{L}}^{\sigma_{\mathcal{L}}, \tau}\left(\exists n \in \mathbb{N}, K_{n} \in T \mid K_{0}=\bar{l}\right)>0$. Since $\mathcal{L}$ is upward-closed then $\overline{\mathcal{L}}$ is downward-closed and since $\mathcal{L}^{\prime \prime}=\emptyset$ then $\sigma_{\mathcal{L}}$ is positively winning in $G_{\mathcal{L}}$ from every non-empty $L^{\prime} \in \overline{\mathcal{L}}$. This proves $(10)$, thus all hypotheses of Lemma 7.3 are satisfied. According to Lemma 7.3 , $\sigma_{\mathcal{L}}$ is almost-surely winning the Büchi game from every initial support in $\overline{\mathcal{L}}$. This terminates the proof of (ii).

The properties of $\mathcal{L}$-games lead to a fix-point characterisation of almost-surely winning supports for player 1.

Proposition 8.7 (FIX-POINT CHARACTERISATION OF ALMOST-SURELY WINNING SUPPORTS). Let $G$ be a Büchi game with observable actions. Let $\mathcal{L} \subseteq \mathcal{P}(K)$ be an upward-closed set of supports such that player 1 can enforce her beliefs to stay outside $\mathcal{L}$. Let $\mathcal{L}^{\prime \prime}$ be the set of supports surely winning for player 2 in the $\mathcal{L}$-game and

$$
\mathcal{L}^{\prime}=\left\{L \notin \mathcal{L} \mid \forall \sigma, \mathbb{P}_{\delta_{L}}^{\sigma, \tau_{\text {rand }}}\left(\exists n, \mathcal{B}_{1}^{n} \in \mathcal{L} \cup \mathcal{L}^{\prime \prime}\right)>0\right\},
$$

where $\tau_{\text {rand }}$ is the strategy for player 2 playing randomly any action. Then,

(i) either $\mathcal{L}^{\prime}=\emptyset$, in this case every support $L \notin \mathcal{L}$ is almost-surely winning for player 1 and her Büchi objective;

(ii) or $\mathcal{L}^{\prime} \neq \emptyset$, in this case:

(a) $\mathcal{L} \cap \mathcal{L}^{\prime}=\emptyset$

(b) player 1 can enforce her beliefs to stay outside $\mathcal{L} \cup \mathcal{L}^{\prime}$,

(c) there is a 2-belief strategy $\tau^{*}$ for player 2 with memory $\mathcal{P}\left(\mathcal{L}^{\prime} \times K\right)$ such that:

$$
\forall \sigma, \forall L \in \mathcal{L}^{\prime}, \mathbb{P}_{\delta_{L}}^{\sigma, \tau^{*}}\left(\mathrm{CoBüchi} \mid \forall n, I_{n} \in \operatorname{ISafe}_{\mathcal{L}}\left(\mathcal{B}_{1}^{n}\right)\right)>0
$$

There exists an algorithm running in time doubly-exponential in the size of $G$ for deciding whether (i) or (ii) holds. In case (ii) holds, the algorithm computes as well $\mathcal{L}^{\prime}$ and $\tau^{*}$.

Proof. We start with proving that if $\mathcal{L}^{\prime \prime}$ is empty then (i) holds. In this case, since player 1 can enforce her beliefs to stay outside $\mathcal{L}$, then $\mathcal{L}^{\prime}$ is empty as well. Moreover, 
according to (ii) of Proposition 8.6, every support not in $\mathcal{L}$ is almost-surely winning for player 1 for the Büchi condition, hence (i) holds.

Suppose now that $\mathcal{L}^{\prime \prime}$ is not empty, Then we prove (ii)(a), (ii)(b) and (ii)(c).

Property (ii)(a) is obvious because $\mathcal{L}^{\prime}$ contains $\mathcal{L}^{\prime \prime}$.

Property (ii)(b) follows from the characterisation in Definition 8.4 if for some $L \in$ $\mathcal{P}(K) \backslash \emptyset$ the set $\operatorname{ISafe}_{\mathcal{L} \cup \mathcal{L}^{\prime}}(L)$ is empty then $\forall \sigma, \mathbb{P}_{\delta_{L}}^{\sigma, \tau_{\text {rand }}}\left(\mathcal{B}_{1}^{1} \in \mathcal{L}^{\prime} \cup \mathcal{L}\right)>0$ thus $L \in \mathcal{L} \cup \mathcal{L}$.

Now we prove (ii)(c). According to (iii) of Proposition 8.6, there exists a 2-belief strategy $\tau^{\prime}$ for player 2 which is surely winning in the $\mathcal{L}$-game from any support in $\mathcal{L}^{\prime \prime}$. We define a 2-belief strategy $\tau^{*}$ for player 2 such that 32 holds. The initial state is $\emptyset$, in this state player 2 throws a coin. As long as the result is "tail", then player 2 plays randomly any action and the memory state is $\emptyset$. If the result is "head" then player 2 picks randomly a memory state $L \in \mathcal{L}^{\prime \prime}$ and switches to the 2-belief strategy $\tau^{\prime}$. Intuitively, player 2 guesses the belief of player 1 , and bets that player 1 will only play safe actions from that moment on. Thus, when playing against $\tau^{*}$, the opponent player 1 does not know whether she faces strategy $\tau^{\prime}$ or strategy $\tau_{\text {rand }}$, because everything is possible with strategy $\tau_{\text {rand }}$.

Let us prove that $\tau^{*}$ guarantees property 32 . By definition of the probability distribution induced by a general strategy, w.l.o.g. it is enough to prove $\sqrt{32}$ in the case where $\sigma$ is a behavioural strategy. Let $L \in \mathcal{L}^{\prime}$ We assume w.l.o.g. that

$$
\mathbb{P}_{\delta_{L}}^{\sigma, \tau^{\prime}}\left(\forall n, I_{n} \in \operatorname{ISafe}_{\mathcal{L}}\left(\mathcal{B}_{1}^{n}\right)\right)>0
$$

otherwise 32 is undefined.

We first prove $(32)$ in case $L \in \mathcal{L}^{\prime \prime}$. By definition of $\mathcal{L}^{\prime \prime}, L$ is surely winning for player 2 in the $\mathcal{L}$-game, and $\tau^{\prime}$ guarantees $\mathbb{P}_{\delta_{L}}^{\sigma, \tau^{\prime}}\left(\operatorname{Win}_{\mathcal{L}}\right)=0$. Since $\operatorname{Win}_{\mathcal{L}}=\left\{\exists n, K_{n} \in T\right.$ and $\forall n, I_{n} \in$ $\left.\operatorname{ISafe}_{\mathcal{L}}\left(\mathcal{B}_{1}^{n}\right)\right\}$ then $\mathbb{P}_{\delta_{L}}^{\sigma, \tau^{\prime}}\left(\exists n, K_{n} \in T \mid \forall n, I_{n} \in \operatorname{ISafe}_{\mathcal{L}}\left(\mathcal{B}_{1}^{n}\right)\right)=0$. There is positive probability that $\tau^{*}$ plays like $\tau^{\prime}$, thus

$$
\mathbb{P}_{\delta_{L}}^{\sigma, \tau^{*}}\left(\exists n, K_{n} \in T \mid \forall n, I_{n} \in \operatorname{ISafe}_{\mathcal{L}}\left(\mathcal{B}_{1}^{n}\right)\right)<1
$$

which implies 32 .

Now we prove (32) in case $L \in \mathcal{L}^{\prime}$. For every $n \in \mathbb{N}$ there is positive probability that $\tau^{*}$ plays like $\tau_{\text {rand }}$ up to step $n$. Thus according to the definition of $\mathcal{L}^{\prime}$,

$$
\mathbb{P}_{\delta_{L}}^{\sigma, \tau^{*}}\left(\exists n, \mathcal{B}_{1}^{n} \in \mathcal{L}^{\prime \prime} \cup \mathcal{L}\right)>0
$$

By definition of $\operatorname{ISafe}_{\mathcal{L}}$, if $\mathcal{B}_{1}^{n} \notin \mathcal{L}$ and $I_{n} \in \operatorname{ISafe}_{\mathcal{L}}\left(\mathcal{B}_{1}^{n}\right)$ this guarantees for sure that $\mathcal{B}_{1}^{n+1} \notin \mathcal{L}$. Since $L \notin \mathcal{L}$ then 35 implies

$$
\mathbb{P}_{\delta_{L}}^{\sigma, \tau^{*}}\left(\exists n, \mathcal{B}_{1}^{n} \in \mathcal{L}^{\prime \prime} \mid \forall n, I_{n} \in \operatorname{ISafe}_{\mathcal{L}}\left(\mathcal{B}_{1}^{n}\right)\right)>0
$$

As a consequence, according to the assumption (33), there exists a finite play $\pi=k_{0} i_{0} j_{0} c_{1} d_{1} k_{1} \cdots k_{n}$ such that $\mathbb{P}_{\delta_{L}}^{\sigma, \tau^{*}}\left(P_{n}=\pi \wedge \forall m, I_{m} \in \operatorname{ISafe}_{\mathcal{L}}\left(\mathcal{B}_{1}^{m}\right)\right)>0$ and $\mathcal{B}_{1}\left(L, c_{1} \ldots c_{n}\right) \in \mathcal{L}^{\prime \prime}$. Denote $B=\mathcal{B}_{1}\left(L, c_{1} \ldots c_{n}\right)$.

Since $\sigma$ and $\tau^{*}$ are behavioural we can apply the shifting lemma (Lemma 7.2) to $\delta_{L}, \sigma, \tau^{*}$ and $E=\left\{\forall m, K_{m} \notin T\right\}$ hence

$$
\mathbb{P}_{\delta_{L}}^{\sigma, \tau^{*}}\left(P_{\geq n} \in E \mid R\right)=\mathbb{P}_{\delta^{\prime}}^{\sigma_{c_{1}} \cdots c_{n}}, \tau_{d_{1} \cdots d_{n}}^{*}(E)
$$

with $R=\left\{C_{1} \cdots C_{n}=c_{1} \cdots c_{n} \wedge D_{1} \cdots D_{n}=d_{1} \cdots d_{n}\right\}$ and $\delta^{\prime}(k)=\mathbb{P}_{\delta_{L}}^{\sigma, \tau^{*}}\left(K_{n}=k \mid R\right)$.

We show that

$$
\operatorname{supp}\left(\delta^{\prime}\right)=B
$$


Since there is positive probability that $\tau^{*}$ plays like $\tau_{\text {rand }}$ for any number of steps, then property (6) of Lemma 7.1 implies $B=\left\{k \in K \mid \mathbb{P}_{\delta}^{\sigma, \tau^{*}}\left(K_{n}=k \mid C_{1} \cdots C_{n}=c_{1} \cdots c_{n}\right)>0\right\}$. Moreover, again because $\tau^{*}$ may play any action at any time whatever signals is received by player $2, \mathbb{P}_{\delta_{L}}^{\sigma, \tau^{*}}\left(K_{n}=k \mid C_{1} \cdots C_{n}=c_{1} \cdots c_{n}\right)>0 \Longleftrightarrow \mathbb{P}_{\delta_{L}}^{\sigma, \tau^{*}}\left(K_{n}=k \mid R\right)>0$. This shows (38).

We have already proved that 32 holds for $L \in \mathcal{L}^{\prime \prime}$ and according to 38 , since $\operatorname{supp}\left(\delta^{\prime}\right)=$ $B \in \mathcal{L}^{\prime \prime}$ we get $\mathbb{P}_{\delta^{\prime}}^{\sigma_{c_{1} \cdots c_{n}}, \tau^{*}}(E)>0$. Since there is positive probability that $\tau_{d_{1} \cdots d_{n}}^{*}$ coincides with $\tau^{*}, \mathbb{P}_{\delta^{\prime}}^{\sigma_{c_{1} \cdots c_{n}}, \tau_{d_{1} \cdots d_{n}}^{*}}(E)>0$. Then (37) implies

$$
\mathbb{P}_{\delta_{L}}^{\sigma, \tau^{*}}\left(P_{\geq n} \in E \mid R\right)>0
$$

By choice of $\pi, \mathbb{P}_{\delta_{L}}^{\sigma, \tau^{*}}\left(P_{n}=\pi \wedge \forall m, I_{m} \in \operatorname{ISafe}_{\mathcal{L}}\left(\mathcal{B}_{1}^{m}\right)\right)>0$ and $\left\{P_{n}=\pi\right\} \subseteq$ $R$ thus $\mathbb{P}_{\delta_{L}}^{\sigma, \tau^{*}}\left(R \mid \forall m, I_{m} \in \operatorname{ISafe}_{\mathcal{L}}\left(\mathcal{B}_{1}^{m}\right)\right)>0$. Together with 39 we get $\mathbb{P}_{\delta_{L}}^{\sigma, \tau^{*}}\left(P_{\geq n} \in E \mid \forall m, I_{m} \in \operatorname{ISafe}_{\mathcal{L}}\left(\mathcal{B}_{1}^{m}\right)\right)>0$. By definition of $E$, it implies

$$
\mathbb{P}_{\delta_{L}}^{\sigma, \tau^{*}}\left(\text { CoBüchi } \mid \forall m, I_{m} \in \operatorname{ISafe}_{\mathcal{L}}\left(\mathcal{B}_{1}^{m}\right)\right)>0
$$

thus $(32)$ is proved.

Description of the algorithm. To terminate the proof of Proposition 8.7, we have to describe the doubly-exponential time algorithm.

First, we compute $\mathcal{L}^{\prime \prime}$ using the algorithm of Proposition 8.6 on the game $G_{\mathcal{L}}$. In case $\mathcal{L}^{\prime \prime}$ is not empty, the algorithm computes $\mathcal{L}^{\prime}$ defined by (31). This can be performed by solving a one-player game with a sure-winning safety condition. The game is a synchronised product of the one-player version of $G$, where player 2 plays totally randomly, with the beliefs of player 1 , this is similar and easier than computing $\mathcal{L}^{\prime \prime}$ and we do not give more details.

Once $\mathcal{L}^{\prime}$ has been computed, the algorithm outputs the 2-belief strategy $\tau^{*}$ with memory $\mathcal{P}\left(\mathcal{L}^{\prime} \times K\right)$, whose construction is described in (ii)(b). For that it uses the algorithm of Proposition 8.6 to output a 2-belief strategy with memory $\mathcal{P}\left(\mathcal{L}^{\prime} \times K\right) \backslash \emptyset$ and adds an initial memory state $\emptyset$ with non-zero transitions probabilities to all other memory states including $\emptyset$ itself.

Now we are done with preliminary results and we turn to the proof of Theorem 8.3 .

Proof of Theorem 8.3. We start with $\mathcal{L}_{0}=\emptyset$ and apply iteratively Proposition 8.7 in order to obtain a sequence $\mathcal{L}_{0}^{\prime}, \mathcal{L}_{1}^{\prime}, \ldots, \mathcal{L}_{M}^{\prime}$ of disjoint non-empty sets of supports such that

—if $1 \leq m \leq M-1$ then $\mathcal{L}_{m}=\mathcal{L}_{0}^{\prime} \cup \cdots \cup \mathcal{L}_{m-1}^{\prime}$ matches case (ii) of Proposition 8.7 which defines a set $\mathcal{L}^{\prime}$ and a strategy $\tau^{*}$ that we rename $\mathcal{L}_{m+1}^{\prime}$ and $\tau_{m+1}^{*}$

$-\mathcal{L}_{M}$ matches case (i) of Proposition 8.7.

Then according to Proposition 8.7, the set of supports positively winning for player 2 is exactly $\mathcal{L}_{M}$, and supports that are not in $\mathcal{L}_{M}$ are almost-surely winning for player 1 .

The sequence $\mathcal{L}_{0}^{\prime}, \mathcal{L}_{1}^{\prime}, \ldots, \mathcal{L}_{M}^{\prime}$ is computable in doubly-exponential time, because each application of Proposition 8.7 involves running the doubly exponential-time algorithm, and the length of the sequence is at most doubly-exponential in the size of the game.

The only thing that remains to prove is the existence and computability of a positively winning 2-belief strategy $\tau^{+}$for player 2. Strategy $\tau^{+}$consists in playing randomly any action as long as a coin gives result "head". When the coin gives result "tail", then strategy $\tau^{+}$chooses randomly an integer $0 \leq m<M$ and a support $L \in \mathcal{L}_{m}^{\prime}$ and switches to strategy $\tau_{m}^{*}$. Intuitively, the strategy bets that the belief of player 1 is exactly $L$ and that, from that moment on, for every step $n$ player 1 will play actions in $\operatorname{ISafe}_{\mathcal{L}_{m}}\left(\mathcal{B}_{1}^{n}\right)$. Since 
each strategy $\tau_{m}^{*}$ has memory $\mathcal{P}\left(\mathcal{L}_{m}^{\prime} \times K\right) \backslash\{\emptyset\}$ and the $\mathcal{L}_{m}^{\prime}$ are distinct, strategy $\tau^{+}$has memory $\mathcal{P}(\mathcal{P}(K) \times K)$ with $\emptyset$ used as the initial memory state.

We prove that $\tau^{+}$is positively winning for player 2 from $\mathcal{L}_{M}$. Let $\sigma$ be a behavioural strategy for player 1 and $L \in \mathcal{L}_{M}$. Let

$$
m_{0}=\min \left\{0 \leq m<M \mid \mathbb{P}_{\delta_{L}}^{\sigma, \tau^{+}}\left(\exists n \in \mathbb{N}, \mathcal{B}_{1}^{n} \in \mathcal{L}_{m}^{\prime}\right)>0\right\}
$$

By minimality of $m_{0}$,

$$
\mathbb{P}_{\delta_{L}}^{\sigma, \tau^{+}}\left(\forall n, I_{n} \in \operatorname{ISafe}_{\mathcal{L}_{m_{0}}}\left(\mathcal{B}_{1}^{n}\right)\right)=1
$$

otherwise, since $\tau^{+}$may play any action at any moment, there would be $n$ such that $\mathbb{P}_{\delta_{L}}^{\sigma, \tau^{+}}\left(\mathcal{B}_{1}^{n+1} \in \mathcal{L}_{m_{0}}\right)>0$. Since $\mathcal{L}_{m_{0}}=\mathcal{L}_{0}^{\prime} \cup \ldots \cup \mathcal{L}_{m_{0}-1}^{\prime}$ this would contradict the minimality of $m_{0}$.

By definition of $m_{0}$, there exists a finite play $p=k_{0} i_{0} j_{0} c_{1} d_{1} \ldots k_{n}$ such that $\mathcal{B}_{1}\left(L, c_{1} \cdots c_{n}\right) \in \mathcal{L}_{m_{0}}^{\prime}$ and $\mathbb{P}_{\delta_{L}}^{\sigma, \tau^{+}}\left(P_{n}=p\right)>0$. Denote $B=\mathcal{B}_{1}\left(L, c_{1}, \ldots, c_{n}\right)$. Since $\sigma$ and $\tau^{+}$are behavioural we can apply the shifting lemma (Lemma 7.2 to $\delta_{L}, \sigma, \tau^{+}$and CoBüchi hence

$$
\mathbb{P}_{\delta_{L}}^{\sigma, \tau^{+}}(\text {CoBüchi } \mid R)=\mathbb{P}_{\delta^{\prime}}^{\sigma_{c_{1} \cdots c_{n}}, \tau_{d_{1} \cdots d_{n}}^{+}} \text {(CoBüchi) }
$$

with $R=\left\{C_{1} \cdots C_{n}=c_{1} \cdots c_{n} \wedge D_{1} \cdots D_{n}=d_{1} \cdots d_{n}\right\}$ and $\delta^{\prime}(k)=\mathbb{P}_{\delta_{L}}^{\sigma, \tau^{+}}\left(K_{n}=k \mid R\right)$. We show that

$$
\operatorname{supp}\left(\delta^{\prime}\right)=B
$$

Since there is positive probability that $\tau^{+}$plays any action at any step then property (6) of Lemma 7.1 implies $B=\left\{k \in K \mid \mathbb{P}_{\delta_{L}}^{\sigma, \tau^{+}}\left(K_{n}=k \mid C_{1} \cdots C_{n}=c_{1} \cdots c_{n}\right)>0\right\}$. Moreover, again because $\tau^{+}$may play any action at any time whatever signals is received by player $2, \mathbb{P}_{\delta_{L}}^{\sigma, \tau^{+}}\left(K_{n}=k \mid C_{1} \cdots C_{n}=c_{1} \cdots c_{n}\right)>0 \Longleftrightarrow \mathbb{P}_{\delta_{L}}^{\sigma, \tau^{+}}\left(K_{n}=k \mid R\right)>0$. This shows 42. Since $\operatorname{supp}\left(\delta^{\prime}\right)=B \in \mathcal{L}_{m_{0}}^{\prime}$, by definition of $\tau_{m_{0}}$, and according to (32) of Proposition 8.7. $\mathbb{P}_{\delta^{\prime}}^{\sigma_{c_{1} \cdots c_{n}}, \tau_{m_{0}}}$ (CoBüchi $\mid \forall n, I_{n} \in \mathrm{ISafe}_{\mathcal{L}_{m_{0}}}$ ) >0. Thus, according to 40, $\mathbb{P}_{\delta^{\prime}}^{\sigma_{c_{1} \cdots c_{n}}, \tau_{m_{0}}}$ (CoBüchi) $>0$. According to the definition of $\tau^{+}$, there is positive probability that $\tau^{+}$switches to strategy $\tau_{m_{0}}$ after signals $d_{1} \cdots d_{n}$ thus

$$
\mathbb{P}_{\delta^{\prime}}^{\sigma_{c_{1} \cdots c_{n}}, \tau_{d_{1} \cdots d_{n}}^{+}} \text {(CoBüchi) }>0 \text {. }
$$

Together with 41 this last inequality implies $\mathbb{P}_{\delta_{L}}^{\sigma, \tau^{+}}($CoBüchi $\mid R)>0$. Moreover $\left\{P_{n}=\right.$ $\pi\} \subseteq R$ and by choice of $\pi, \mathbb{P}_{\delta_{L}}^{\sigma, \tau^{+}}\left(P_{n}=\pi\right)>0$ thus $\mathbb{P}_{\delta_{L}}^{\sigma, \tau^{+}}$(CoBüchi) $>0$. Since this holds for any behavioural strategy $\sigma$, the strategy $\tau^{+}$is positively winning from any $L \in \mathcal{L}_{M}$.

\section{LOWER BOUND ON MEMORY NEEDED BY STRATEGIES.}

In this section, we give the proof of the lower bound in Theorem 6.6, stating that doubly exponential memory is necessary to win positively. This lower bound holds for both finite-memory strategies with randomised updates and for finite-memory strategies with deterministic updates. This should be compared the doubly exponential upper bound of Theorem 6.6. obtained for strategies for player 2 with randomised updates, built from the fix point algorithm of the previous section.

\subsection{Overview of the proof}

We show in this section that a doubly-exponential memory is necessary to win positively safety (and hence co-Büchi) games. 
To this aim, we construct, for each integer $n$, a reachability game of size polynomial in $n$. A high-level description of this game, called guess_my_set ${ }_{n}$, is given in Fig. 10. The objective of player 1 is to reach $\odot$, while player 2 has the dual objective of avoiding $\odot$. We will establish that player 2 wins positively, and that the memory of any positively winning strategy for player 2 is at least doubly exponential in $n$. These properties are postponed to Proposition 9.1 .

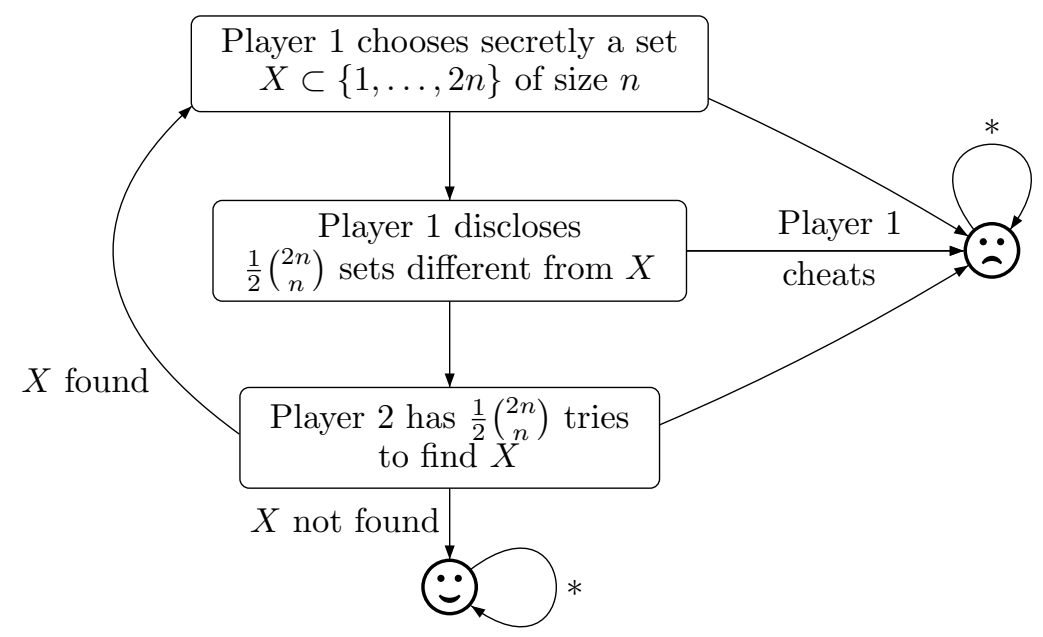

Fig. 10. A game where player 2 needs doubly-exponential memory to avoid the $\odot$-state with positive probability.

Let us start by describing the high-level structure of guess_my_set ${ }_{n}$ for a fixed $n \in \mathbb{N}$.

Idea of the game. The game guess_my_set ${ }_{n}$ is divided into three phases, represented by blocks in Fig. 10. In the first phase, player 1 chooses a set $X \subsetneq\{1, \ldots, 2 n\}$ of size $n$. There are $\left(\begin{array}{c}2 n \\ n\end{array}\right)$ possibilities of such sets $X$. Player 2 is blind in this phase and has no action to play.

In the second phase, player 1 discloses through her actions $\frac{1}{2}\left(\begin{array}{c}2 n \\ n\end{array}\right)$ pairwise distinct sets of size $n$ which are all different from $X$. Player 2 has no action to play in that phase, yet he observes the actions of player 1 and thus the sets disclosed by player 1 .

In the third phase, player 2 aims at guessing $X$ by trying up to $\frac{1}{2}\left(\begin{array}{c}2 n \\ n\end{array}\right)$ sets of size $n$. Similarly to player 1 in phase 2, here player 2 discloses these sets by his actions. In this phase, player 1 has no action to play, yet she observes actions of her opponent. If player 2 succeeds in guessing $X$, the game restarts from the beginning. Otherwise, state $;$ is reached and player 1 wins.

In order for guess_my_set ${ }_{n}$ to be of polynomial size in $n$, the various sets $X$, and the ones disclosed by player 1 , or tried by player 2 , cannot be stored in the arena. A consequence of this is to allow player 1 to cheat: either in the first phase by picking a set of size not equal to $n$, or in the second phase by disclosing set $X$, or in the third phase by pretending player 2 did not guess $X$. To prevent player 1 from cheating, we rely on probabilities and store in the state of the game a short random information, e.g., one element of $X$ as opposed to the whole set. Therefore, if player 1 cheats, she will be caught with positive probability, yielding to a sink losing state $\odot$. To win almost-surely, player 1 will thus have to play according to the rules. Our concise encoding however will not allow player 2 to cheat. Notice that player 1 is better informed than player 2 in this game. 
Concise encoding. Let us explain in more details the encoding of the game guess_my_set $_{n}$, to justify that its number of states is polynomial in $n$. There are three issues to be addressed. First, storing set $X$ in the state of the game would require exponentially many states. Instead, we use a fairly standard technique: store a single element $x \in X$ at random. In order to check that a set $Y$ of size $n$, disclosed by player 1, is different from $X$, we challenge player 1 to pinpoint an element $y \in Y \backslash X$. We ensure by construction that $y \in Y$ : player 1 has to pinpoint $y$ when disclosing $Y$. If player 1 cheats in phase 2 , she pinpoints $y \in X$, and with positive probability $y=x$, in which case the game moves to $\odot$ and player 1 loses.

The second issue is to make sure that player 1 discloses an exponential number of pairwise different sets $X_{1}, X_{2}, \ldots, X_{\frac{1}{2}\left(\begin{array}{c}2 n \\ n\end{array}\right)}$, while the game cannot store even one of these sets. Instead, player 1 will disclose the sets in some total order, denoted $<$. Thus it will suffice to check only one inequality each time a set $X_{i+1}$ is given, namely $X_{i}<X_{i+1}$. The precise encoding is more involved than with the previous issue, but relies on similar ideas (see subsection 9.3 .

The last issue is to count up to $\frac{1}{2} \cdot\left(\begin{array}{c}2 n \\ n\end{array}\right)$, with a logarithmic number of bits, to check that that number of sets have been disclosed by player 1 , or tried by player 2 . Here again, we ask player 1 to increment a counter, while storing only one of the bits. If she is caught cheating when incrementing the counter, the game moves to $:$ (see subsection 9.2).

Now that we gave a high-level description of the game, we can state its properties:

Proposition 9.1. Player 2 has a positively winning finite-memory strategy with deterministic updates with $3 \times 2^{\frac{1}{2} \cdot\left(\begin{array}{c}2 n \\ n\end{array}\right)}$ different memory states in the game guess_my_set ${ }_{n}$.

No finite-memory strategy with randomised updates of player 2 with less than $2^{\frac{1}{2} \cdot\left(\begin{array}{c}2 n \\ n\end{array}\right)}$ memory states wins positively guess_my_set $_{n}$.

Proof. Let us describe a positively winning strategy for player 2 with at most $3 \times$ $2^{\frac{1}{2} \cdot\left(\begin{array}{c}2 n \\ n\end{array}\right)}$ memory states. First of all, player 2 remembers the phase the game is (3 different possibilities). In phase 2 , player 2 remembers all the sets disclosed by player $1\left(2^{\frac{1}{2} \cdot\left(\begin{array}{c}2 n \\ n\end{array}\right)}\right.$ possibilities). Between phase 2 and phase 3 , it reverses his memory to remember the sets player 1 did not disclose ( $\operatorname{still} 2^{\frac{1}{2} \cdot\left(\begin{array}{c}2 n \\ n\end{array}\right)}$ possibilities). Then he tries each of these sets, one by one, in phase 3 , deleting the set from his memory after he tried it.

Let us assume first that player 1 does not cheat. Then each set of size $n$ is either disclosed by player 1 , or tried by player 2 , since there are $\left(\begin{array}{c}2 n \\ n\end{array}\right)$ such sets. As a consequence, $X$ has been found, and game starts another round, and avoids $(-)$. Else, if player 1 cheats at some point, there is a positive probability to reach the losing state $\odot$, and player 2 also wins positively his safety objective.

To show the second claim, assume by contradiction that there exists a positively winning

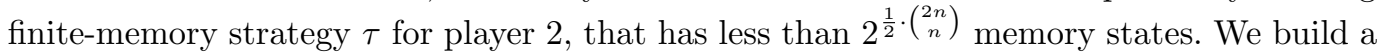
counter strategy $\sigma$ to $\tau$ for player 1 . Note that $\sigma$ shall not cheat, else the game would enter the sink losing state with positive probability. Strategy $\sigma$ actually takes all its decisions at random: it chooses the secret set $X$ at random in phase 1; then in phase 2, it chooses pairwise distinct sets $Y \neq X$ uniformly at random, and discloses them following the total order. At the end of phase 2 for each round of the game, player 1 has disclosed a family $\mathcal{A}$ of $\frac{1}{2} \cdot\left(\begin{array}{c}2 n \\ n\end{array}\right)$ sets of size $n$. The distribution over memory states of player 2 at that moment only depends on $\mathcal{A}$ and on the distribution of his memory state at the beginning of the round. Let us fix a round (thus the initial distribution over memory states of $\tau$ is fixed) and denote by $m_{\mathcal{A}}$ the distribution of memory states of player 2 after $\mathcal{A}$ has been disclosed. As $\tau$ has less than $2^{\frac{1}{2} \cdot\left(\begin{array}{c}2 n \\ n\end{array}\right)}$ memory states, there exists at least one memory state $\mathfrak{m}$ and two families $\mathcal{B} \neq \mathcal{C}$ such that $m_{\mathcal{B}}(\mathfrak{m}) \neq 0$ and $m_{\mathcal{C}}(\mathfrak{m}) \neq 0$. Let $\overline{\mathcal{B}}($ resp. $\overline{\mathcal{C}}$ ) be the complement 
of $\mathcal{B}$ (resp. $\mathcal{C}$ ) among the set of sets of $n$ elements. Since $\mathcal{B} \neq \mathcal{C}, \bar{B} \cup \bar{C}$ has strictly more than $\frac{1}{2} \cdot\left(\begin{array}{c}2 n \\ n\end{array}\right)$ sets of $n$ elements. Hence, there exists a set $Y \in \overline{\mathcal{B}} \cup \overline{\mathcal{C}}$ which is tried by player 2 with probability less than 1 after memory state $\mathfrak{m}$. Without loss of generality, we can assume that $Y \notin \mathcal{B}$ (the case $Y \notin \mathcal{C}$ is symmetrical). The probability is non zero that player 1 chose set $Y$ in the first phase of that round, and discloses $\mathcal{B}$. Hence there is a non zero probability that player 2 does not try set $Y$ in phase 3 , in which case $(-)$ is reached in that round. More precisely, there is a uniform lower bound $p>0$ on the probability to reach $(;)$ at each round. As it is true for every round, almost-surely $(;)$ is reached, a contradiction with the fact that $\tau$ is positively winning. Thus, no finite-memory strategy for player 2 with less than $2^{\frac{1}{2} \cdot\left(\begin{array}{c}2 n \\ n\end{array}\right)}$ memory states can be positively winning.

\subsection{Concise encoding of exponentially many steps}

As a first step to the formal definition of guess_my_set $_{n}$, we explain how to concisely count up to a number exponential in $n$ with only a number of states polynomial in $n$.

Let $y_{1} \cdots y_{n}$ be the binary encoding of a number $y$ exponential in $n$, where $y_{n}$ is the parity of $y$. We describe a single player reachability game, in which the player surely wins if the game lasts for $n \cdot y$ steps. Intuitively, the player increments a counter from 0 to $y_{1} \cdots y_{n}$. For a counter value $x$, let $x_{1}^{\prime} \cdots x_{n}^{\prime}$ be the binary encoding of $x^{\prime}=x+1$. In order to check that the player does not cheat in the incrementation step, some bit $x_{i}^{\prime}$ for a random $i$ is stored in the game state, and hidden to the player. The value of $x_{i}^{\prime}$ can easily be computed on the fly while reading $x_{i} \ldots x_{n}$ : indeed, $x_{i}^{\prime}=x_{i}$ iff there exists some $k>i$ with $x_{k}=0$.

In this game, the set of signals is the same as the set of actions, namely $\{0,1,2\}$. Actions $a \in\{0,1\}$ stand for the value of bits, while $a=2$ represents that the player claims to have reached $y$. The state space is the following: $\left\{\left(i, b, j, b^{\prime}, j^{\prime}, c\right) \mid\left(i, j, j^{\prime}\right) \in\right.$ $\left.\{1, \cdots, n\}^{3},\left(b, b^{\prime}, c\right) \in\{0,1\}^{3}\right\}$. The intuition of such a state is that the player will play action $a_{i}$ corresponding to bit $x_{i}$, while $b, j$ is the check to make to the current number (checking that $\left.x_{j}=b\right), b^{\prime}, j^{\prime}$ is the check to make to the successor of $x\left(x_{j^{\prime}}^{\prime}=b^{\prime}\right)$, and $c$ indicates whether there is a carry (correcting $b^{\prime}$ in case $c=1$ at the end of the current number $(i=n)$. The initial distribution is the uniform distribution on $(0,0, k, 0,1)$ (checking that the initial number generated is indeed 0 ). If the player plays action 2, claiming that $y$ has been reached, then if $y_{j} \neq b$, the player is caught cheating (since the current counter value is certainly not $y$ ), and the game moves to a losing sink state $\odot$. Otherwise, when $y_{j}=b$, the game moves to the goal state $\odot$. Thus, there is a transition in the arena with $p\left(\left(i, b, j, b^{\prime}, j^{\prime}, c\right), a, \odot\right)=1$ if $i=j$ and $a \neq b$, corresponding to the player being caught cheating. Else, if $i \neq n$, the stochastic transitions are

- The current bit $a$ at position $i$ may be checked for the successor $x^{\prime}$ of $x$ : $p\left(\left(i, b, j, b^{\prime}, j^{\prime}, c\right), a,(i+1, b, j, a, i, 1)\right)=1 / 2$ (carry initialised at 1$)$, and

- The current bit will not be checked: $p\left(\left(i, b, j, b^{\prime}, j^{\prime}, c\right), a,\left(i+1, b, j, b^{\prime}, j^{\prime}, c \wedge a\right)\right)=\frac{1}{2}$ (the carry is 1 if both $c$ and $a$ are 1).

Last, for $i=n$, there is a transition $p\left(\left(i, b, j, b^{\prime}, j^{\prime}, c\right), a,\left(1, b^{\prime} \wedge c, j^{\prime}, a, 1,1\right)\right)=1$ (the bit of the next number becomes the bit for the current configuration, taking care of the carry $c$ ). Clearly, if the game does not last $n \cdot y$ steps, then the player did not faithfully encode the counter increment at some step, and she has a chance to get caught and lose, so that the probability to reach $(\dot{)}$ is less than 1 .

\subsection{Implementing guess_my_set ${ }_{n}$ with a polynomial size game.}

We finally turn to the formal definition of the game guess_my_set $_{n}$, with a number of states polynomial in $n$. Recall that player 1 has a reachability objective, namely the target state (-). 
In the first phase of each round, player 1 chooses a set $X$ of $n$ elements in $\{1, \cdots 2 n\}$. Formally, each number from 1 to $2 n$ is called in increasing order, and player 1 has two actions, "yes" or "no", to define the set $X$. She has to play "yes" for exactly $n$ numbers. The states of that phase of the game are of the form $(x, i, r)$, where $x$ is the number currently called, $i$ counts the number of "yes" actions so far, and $r$ is some element for which player 1 played "yes", that the system stores, and which is hidden to both players. Signals of player 1 coincide with her actions. Player 2 does not participate in this phase: his actions have no effect on the state and he receives always the same dummy signal whatever happens.

Formally, whenever player 1 plays "yes" for a number $x$, there are two stochastic transitions $p((x, i, r)$, yes,$(x+1, i+1, x))=1 / 2$ and $p((x, i, r)$, yes, $(x+1, i+1, r)=1 / 2$. In both cases $x$ is selected as the $i+1$-th number in set $X$, the current size of $X$ is increased by 1 , and the next number called is $x+1$. In the former case, the randomly stored number $r$ is updated to $x$, while in the latter case $r$ is not updated. The stored number $r$ at the end of phase 1 will be used in the other phases of this round. If player 1 plays action "no" for a number $x$, this triggers the transition $p((x, i, r), n o,(x+1, i, r))=1$.

State $(2 n+1, i, r x)$ with $i \neq n$ encodes that player 1 did not select with "yes" actions exactly $n$ numbers, and the game moves directly to the sink losing state $\odot$.

In the second phase, player 1 discloses $\frac{1}{2} \cdot\left(\begin{array}{c}2 n \\ n\end{array}\right)$ distinct sets $Y$ of size $n$, all different of $X$. In order to be sure that every set $Y$ she proposes is different from $X$, player 1 is asked to pinpoint an element in $Y \backslash X$. This number is not visible to player 2. In case player 1 pinpoints $y$ equal to the stored number $r$, which belongs to $X$ by construction, then she is caught cheating, and the game moves to the sink losing state $\odot$. Since player 1 does not know the number $r$, pinpointing any number in $X$ is risky as it makes her lose with fixed positive probability.

To force player 1 to disclose distinct sets $Y$, she enumerates them in lexicographic order $<$. Formally, $Y<Y^{\prime}$ if there exists a position $i$ such that the $i$-1-th smallest numbers of $Y$ and of $Y^{\prime}$ agree, and the $i$-th smallest number $y$ of $Y$ is less than the $i$-th smallest number of $Y^{\prime}$. The number $y$ is called the distinguishing number between $Y$ and $Y^{\prime}$. To check that player 1 generates sets in lexicoraphic order, when disclosing $Y$, player 1 must announce which is the distinguishing number $y$ between $Y$ and $Y^{\prime}$, for $Y^{\prime}$ the next disclosed set. The actions of player 1 relevant to ensure that sets are enumerated in lexicographic order and distinct are thus $\{$ yesdif, yes, no $\}$. Similarly to the first phase, one such action is played for each number in $\{1, \cdots 2 n\}$ when they are called. Intuitively, yesdif represents that $y$ the number being called belongs to $Y$ and is the distinguishing number between $Y$ and the next set $Y^{\prime}$. Else, yes represents that $y \in Y$ and no stands for $y \notin Y$.

Formally, the part of the states relevant to ensure that the disclosed sets are distinct is of the form $(y, i, p r y, p y, p i, n r y, n y, n i)$, with:

- $y$ the number being called,

- $i$ the number of yes played by player 1 so far for the current set $Y^{\prime}$,

- $p y, p i$ the distinguishing number and its position as announced for the previous set $Y$,

- pry a random number in $Y$ and less than py (hidden to both players),

- ny, ni the distinguishing number and its position for the current set $Y^{\prime}$ (initially 0,0 ), and

- nry a random number in $Y$ less than $n y$ (hidden to both players).

The stored numbers (py, pi,pry) yield a chance to catch player 1 if she does not generate $Y$ in lexicographic order, in which case the game is sent to the sink state $\odot \cdot$. First, the game checks that the $p i$-th number in $Y$ is larger than $p y$. Also, the game checks that pry belongs to the current set. Hence, player 1 needs to ensure that the first pry -1 elements of the previous set and of the current set agree, and that the the $p i$-th number in $Y$ is larger than $p y$, that is $Y$ is larger lexicographically than the previous set. Otherwise, she has a fixed positive probability to be caught, and she will lose the game in $:-$ 
In this phase, player 2 has no actions to play. The signals of player 1 and 2 are the same as the actions of player 1, that is, player 2 is informed of the sets disclosed by player 1 .

Formally, the transitions depend on whether player 1 is caught cheating using $(y, i, p r y, p y, p i)$ or not. She is caught cheating using $(y, i, p r y, p y, p i)$ with the following transitions, with yes $*=$ yes or yes $*=$ yesdif:

$-p((y, i, p r y, p y, p i), y e s *, \odot)=1$ for $y=p y$,

$-p((y, i, p r y, p y, p i), y e s *, \odot)=1$ for $i=p i-1$ and $y \geq p y$.

Else, we have the transitions:

$-p((y, i, n r y, n y, n i), a, \odot)=1$ for $n y \neq 0$ and $a=y e s d i f$, corresponding to player 1 announcing two distinguishing numbers, or for $y=2 n$, ny $=0$ and $a \neq$ yesdif, corresponding to no distinguishing numbers. Otherwise,

- for $a=n o, p((y, i, n r y, n y, n i), n o,(y+1, i, n r y, n y, n i))=1$, because when $y$ does not belong to the current set, nothing has to be updated and the next number $y+1$ is called.

- for $a=$ yesdif, $p\left((y, i, n r y, n y, n i)\right.$, yesdif,$\left.\left(y+1, i+1, n r y, n y^{\prime}, n i^{\prime}\right)\right)=1$ with $n y^{\prime}=y$ and $n i^{\prime}=i$, because player 1 just pinpointed the number called with yesdif,

- for $a=y e s$ and $n y>0$, meaning that the distiguishing number was already pinpointed, $p((y, i, n r y, n y, n i)$, yes, $(y+1, i+1, n r y, n y, n i))=1$,

- else, $a=$ yes and $n y=0$, meaning that no distinguishing number was pinpointed yet, and then we have $p((y, i, n r y, n y, n i), a,(y+1, i+1, n r y, n y, n i))=1 / 2$ and $p\left((y, i, n r y, n y, n i), a,\left(y+1, i+1, n r y^{\prime}, n y, n i\right)\right)=1 / 2$ with $n r y^{\prime}=y$. This corresponds to the fact that $y \in Y$ and $y$ is less than the distinguishing number, so that it can be randomly chosen to be stored in nry.

Last, to ensure that player 1 discloses $\frac{1}{2} \cdot\left(\begin{array}{c}2 n \\ n\end{array}\right)$ sets, she has to encode the increment of a counter, as described in Subsection 9.2 , where the counter is incremented exactly when a set $Y$ is disclosed. When she has given $\frac{1}{2} \cdot\left(\begin{array}{c}2 n \\ n\end{array}\right)$ sets, the game proceeds to the third phase.

In the third phase, player 2 has at most $\frac{1}{2} \cdot\left(\begin{array}{c}2 n \\ n\end{array}\right)$ tries to guess the set $X$ chosen by player 1 in the first phase. To do so, player 2 tries $\frac{1}{2} \cdot\left(\begin{array}{c}2 n \\ n\end{array}\right)$ sets of size $n$, and player 1 observes these sets. For each set $Y$ tried by player 2, player 1 has to announce a witness in $Y \backslash X$, which is not observed by player 2 . Similarly to phases 1 and 2 , numbers in $\{1, \cdots 2 n\}$ are called in increasing order, and player 2 plays yes or no to tell whether they belong to $Y$. Just after player 2 announces that $y$ is in the guessed set $Y$, player 1 can announce secretly that " $y \in Y \backslash X$ ". Player 1 is caught cheating if $y$ coincides with $r$ the stored number from the first phase. If $Y=X$, player 1 cannot announce $y \in Y \backslash X$ without having a chance to be caught. Instead, she can play a reset action to restart the game in its first phase. Note that player 1 only has an incentive to play that reset action in case $Y \neq X$, since otherwise, she would rather select $y \in Y \backslash X$. After each set tried by player 2 , the counter, as described in section 9.2 is incremented. When $\frac{1}{2} \cdot\left(\begin{array}{c}2 n \\ n\end{array}\right)$ sets $Y \neq X$ have been tried by player 2 without guessing $X$, the game moves to winning state $\odot$, and player 1 wins.

\section{COMPLEXITY LOWER-BOUND AND SPECIAL CASES.}

In this section we show that our 2EXPTIME algorithms are optimal regarding complexity. Furthermore, we show that these algorithms enjoy better complexity in restricted cases. In particular, we generalise a result of [Reif 1979, Chatterjee et al. 2007], extending EXPTIME complexity to a larger subclass of systems with particular signalling structures, as described in Section 10.2 . 


\subsection{Complexity lower bound for reachability and Büchi games.}

We prove here that the problem of knowing whether the initial support of a reachability game or a Büchi game is almost-surely winning for player 1 is 2EXPTIME-complete. The lower bound even holds when player 1 is more informed than player 2 .

THEOREM 10.1. In a reachability or Büchi game, deciding whether player 1 has an almost-surely winning strategy is 2EXPTIME-hard, even if player 1 is more informed than player 2 .

We provide a proof for reachability games. The lower-bound of course extends to Büchi games since any reachability game can be turned into an equivalent Büchi one by making target states absorbing.

Proof. We do a reduction from the membership problem for EXPSPACE alternating Turing machines. Let $\mathcal{M}$ be an EXPSPACE alternating Turing machine, and $w$ be an input word of length $n$. From $\mathcal{M}$ and $w$ we build a stochastic game with partial observation such that player 1 can achieve almost-surely a reachability objective if and only if $w$ is accepted by $\mathcal{M}$. The idea of the game is that player 2 describes an execution of $\mathcal{M}$ on $w$, that is, he enumerates the tape contents of successive configurations. Moreover he chooses the rule to apply when the state of $\mathcal{M}$ is universal, whereas player 1 is responsible for choosing the rule in existential states. When the Turing machine reaches its final state, the play is won by player 1. Both players will be able to deviate from these rules, but then they will have a non zero probability to be caught cheating, immediately ending the game in a state where the other player wins. In this game, if player 2 implements some execution of $\mathcal{M}$ on $w$ without cheating, player 1 has a surely winning strategy if and only if $w$ is accepted by $\mathcal{M}$. Indeed, if all executions on $w$ reach the final state of $\mathcal{M}$, then whatever the choices player 2 makes in universal states, player 1 can properly choose rules to apply in existential states in order to reach a final configuration of the Turing machine. On the other hand, if some execution on $w$ does not lead to the final state of $\mathcal{M}$, player 1 is not sure to reach a final configuration.

This reasoning holds under the assumption that player 2 effectively describes the execution of $\mathcal{M}$ on $w$ consistent with the rules chosen by both players. However, player 2 could cheat when enumerating successive configurations of the execution. He would for instance do so, if $w$ is indeed accepted by $\mathcal{M}$, in order to have a chance not to lose the game. To prevent player 2 from cheating (or at least to prevent him from cheating too often), it would be convenient for the game to remember the tape contents, and check that in the next configuration, player 2 indeed applied the chosen rule. However, the game can remember only a logarithmic number of bits, while the configurations have a number of bits exponential in $n$. Instead, a position $k$ of the tape is chosen at random, and is revealed to player 1 as a sequence of $n$ bits. Player 2 is not told anything about $k$. The game stores the letter at this position together with the previous and next letter on the tape. This allows the game to compute the letter $a$ at position $k$ of the next configuration. As player 2 describes the next configuration, player 1 should announce to the game that position $k$ has been reached again (player 1 can cheat by announcing a different $k^{\prime} \neq k$ - but we will first assume it is not the case). The game checks that the letter player 2 gives is indeed $a$. This way, each time player 2 cheats, the game has a fixed positive probability to detect it. If so, the game goes to a sink state which is winning for player 1 .

On top of that, player 1 has the possibility to reset the whole execution whenever she wants and restart a fresh computation.

Assuming that $w$ is accepted by $\mathcal{M}$, we show that player 1 wins almost-surely. Consider a strategy where player 1 does not cheat, plays a strategy ensuring that the computation on $w$ is accepting, and resets as soon as player 2 cheats and the system does not detect it. There are two kinds of plays: those where player 2 plays fair during at least one whole computation, and those where player 2 cheats at least once after each reset. In the first 
case, the computation on $w$ terminates in an accepting state. In the second case, player 2 gets caught almost-surely: each time player 2 cheats, there is probability at least $\frac{1}{2^{n}}$ that player 2 gets caught (the probability that $k$ chosen by the system is the cheating position). In both cases player 1 wins.

We now have to take into account that player 1 could cheat: she could call to a position different from $k$ in the next step. To avoid this kind of behaviour, or at least refrain it, a piece of information about the position pointed by player 1 is kept secret (to both players) in the state of the game. More precisely, a bit $b$ of the binary encoding of $k$ is randomly chosen among the at most $n$ possible bits, and the bit and its position are remembered. If player 1 is caught cheating (that is, if the bits at the position remembered differ between both step), the game goes to a sink state losing for player 1 . This way, when player 1 decides to cheat, there is a positive probability that she loses the game.

Assume that $w$ is not accepted by $\mathcal{M}$, we show that player 2 wins positively. For that player 2 plays a strategy not cheating and ensuring $w$ is not accepted by $\mathcal{M}$. Either player 1 cheats and has probably $<1$ to win, or she does not cheat and has probability 0 to win.

Finally, $w$ is accepted by $\mathcal{M}$ if and only if player 1 has an almost-surely winning strategy to reach the goal state.

Notice that the game is stochastic (a bit and a position are remembered randomly in states of the game), player 1 is not perfectly informed about the state (she does not know which bit is remembered in the state), but she is better informed than player 2 (the latter does not know what letter player 1 decided to memorise).

Finally, let us comment on the almost-surely winning strategy of player 1 built in the proof. This strategy requires a doubly-exponential number of memory states for detecting whenever player 2 is cheating. This contrasts with our exponential upper-bound on the memory needed by player 1 to win almost-surely. Actually player 1 has a simpler strategy to win almost-surely: it is enough for her to play almost totally randomly. Resets are triggered randomly and the existential choices of the computation of the machine are also performed randomly. Only one thing should be done with care by player 1: she should remember exactly the value of the position $k$ in order to announce it accurately when the next configuration occurs. This requires exponentially many memory states.

\subsection{Special cases.}

A first straightforward result is that in a safety game where player 1 has full information, deciding whether she has an almost-surely winning strategy is in PTIME.

Now, consider a Büchi game. In general, as shown in the previous section, deciding whether the game is almost-surely winning for player 1 is 2EXPTIME-complete. In Chatterjee et al. 2007, it is shown that this problem is EXPTIME-complete when player 2 is perfectly informed. The following proposition shows that actually player 2 being better informed than player 1 is a sufficient condition for the complexity to drop from 2EXPTIME to EXPTIME. Orthogonally, player 1 being perfectly informed about the state is also sufficient to obtain EXPTIME complexity.

Proposition 10.2. In a Büchi game where either player 2 is better informed than player 1 or player 1 is perfectly informed about the state, deciding whether player 1 has an almost-surely winning strategy can be done in exponential time.

Proof. The reason for the single EXPTIME complexity in these special cases is that in both cases, there are at most an exponential number of 2-beliefs for player 2 . If player 1 is perfectly informed about the state, then the belief of player 1 is a singleton $\{k\}$. Thus the 2-belief of player 2 is a collection of pairs $(k,\{k\})$ with $k \in K$. There is an exponential number of such 2-beliefs. 
If player 2 is better informed than player 1 , then at every moment player 2 can compute exactly the belief $\mathcal{B}_{1}$ of player 1 . Thus the 2-belief of player 2 is a collection $\left\{\left(k, \mathcal{B}_{1}\right), k \in K^{\prime}\right\}$ with $K^{\prime} \subseteq K$ (actually $K^{\prime} \subseteq \mathcal{B}_{1}$ ). For a given value of $\mathcal{B}_{1}$ there are at most $2^{|K|}$ such collections thus in total there are less than $2^{2|K|}$ possible 2-beliefs.

Note that the latter proposition does not hold when player 1 is better informed than player 2. Indeed in the game presented for the lower-bound, in the proof of Theorem 10.1 . player 1 is better informed than player 2 (yet player 1 is not perfectly informed about the state).

\section{CONCLUSION.}

We considered stochastic games with signals and established two determinacy results. First, a reachability game is either almost-surely winning for player 1 , surely winning for player 2 or positively winning for both players. Second, a Büchi game is either almost-surely winning for player 1 or positively winning for player 2 . We gave algorithms for deciding in doublyexponential time which case holds and for computing winning strategies with finite memory. Further, we showed that both the memory and the algorithmic complexities are tight.

Changing the notion of reaching a Büchi objective with positive probability for the notion where the frequency at which a target state is visited does not converge towards 0 leads to decidability of the emptiness problem of probabilistic finite automaton [Tracol 2011] . It would be interesting to extend this result to stochastic games with signals.

\section{ACKNOWLEDGMENTS}

We thank the anonymous referees for their much valuable comments which allowed us to significantly improve this paper. In particular, one of the referees found a flaw in an earlier version, which led us to establish the equivalence between games with non-observable actions and general strategies and games with observable actions and behavioural strategies.

\section{REFERENCES}

Robert J. Aumann. 1964. Mixed and Behavior Strategies in Infinite Extensive Games. In Advances in Game Theory, Annals of Mathematics Studies, USA: Princeton University Press (Ed.), Vol. 52. MIT Press, $627-650$.

Robert J. Aumann. 1995. Repeated Games with Incomplete Information. MIT Press.

C. Baier, N. Bertrand, and M. Größer. 2008. On Decision Problems for Probabilistic Büchi Automata. In Proc. of FOSSACS'08 (LNCS), Vol. 4972. Springer, 287-301.

Nathalie Bertrand, Blaise Genest, and Hugo Gimbert. 2009. Qualitative Determinacy and Decidability of Stochastic Games with Signals. In LICS. IEEE Computer Society, 319-328.

D. Berwanger, K. Chatterjee, L. Doyen, T. A. Henzinger, and S. Raje. 2008. Strategy Construction for Parity Games with Imperfect Information. In Proc. of CONCUR'08 (LNCS), Vol. 5201. Springer, 325-339.

Tomás Brázdil, Václav Brozek, Antonín Kucera, and Jan Obdrzálek. 2011. Qualitative reachability in stochastic BPA games. Inf. Comput. 209, 8 (2011), 1160-1183. DOI : http://dx.doi.org/10.1016/j.ic.2011.02.002

Krishnendu Chatterjee, Luca de Alfaro, and Thomas A. Henzinger. 2005. The Complexity of Stochastic Rabin and Streett Games. In Proc. of ICALP'05 (LNCS), Vol. 3580. Springer, 878-890.

Krishnendu Chatterjee and Laurent Doyen. 2012. Partial-Observation Stochastic Games: How to Win when Belief Fails. In Proc. of LICS'12. IEEE, 175-184. Long version available at http://arxiv.org/abs/1107. 2141

Krishnendu Chatterjee, Laurent Doyen, Hugo Gimbert, and Thomas A. Henzinger. 2010. Randomness for Free. In Proc. of MFCS'10 (LNCS), Vol. 6281. Springer, 246-257.

Krishnendu Chatterjee, Laurent Doyen, and Thomas A. Henzinger. 2013. A survey of partial-observation stochastic parity games. Formal Methods in System Design 43, 2 (2013), 268-284.

K. Chatterjee, L. Doyen, T. A. Henzinger, and J.-F. Raskin. 2007. Algorithms for Omega-regular Games of Incomplete Information. Logical Methods in Computer Science 3, 3 (2007). 
Krishnendu Chatterjee, Marcin Jurdzinski, and Thomas A. Henzinger. 2004. Quantitative stochastic parity games. In Proc. of SODA'04. SIAM, 121-130.

A. Condon. 1992. The complexity of stochastic games. Information and Computation 96 (1992), 203-224.

Julien Cristau, Claire David, and Florian Horn. 2010. How do we remember the past in randomised strategies?. In Proceedings First Symposium on Games, Automata, Logic, and Formal Verification, GANDALF 2010, Minori (Amalfi Coast), Italy, 17-18th June 2010. 30-39. DOI: http://dx.doi.org/10.4204/EPTCS.25.7

L. de Alfaro and T. A. Henzinger. 2000. Concurrent Omega-Regular Games. In Proc. of LICS'00. IEEE, $141-154$.

Luca de Alfaro, Thomas A. Henzinger, and Orna Kupferman. 2007. Concurrent reachability games. Theoretical Computer Science 386, 3 (2007), 188-217.

L. de Alfaro and R. Majumdar. 2001. Quantitative Solution of Omega-Regular Games. In Proc. of STOC'01. ACM, 675-683.

Rick Durrett. 2010. Probability: Theory and Examples (4th ed.). Cambridge University Press.

Hugo Gimbert and Florian Horn. 2008. Simple Stochastic Games with Few Random Vertices Are Easy to Solve. In Proc. of FOSSACS'08 (LNCS), Vol. 4972. Springer, 5-19.

Hugo Gimbert and Youssouf Oualhadj. 2010. Probabilistic Automata on Finite Words: Decidable and Undecidable Problems. In Proc. of the 37th International Colloquium on Automata, Languages and Programming (ICALP '10) (Lecture Notes in Computer Science), Vol. 6199. Springer, 527-538.

Hugo Gimbert, Jrme Renault, Sylvain Sorin, Xavier Venel, and Wiesław Zielonka. 2016. On the values of repeated games with signals, with signals. Annals of Applied Probability 26, 1 (February 2016), 402-424.

E. Grädel, W. Thomas, and T. Wilke. 2002. Automata, Logics and Infinite Games. LNCS, Vol. 2500. Springer.

Vincent Gripon and Olivier Serre. 2009. Qualitative Concurrent Stochastic Games with Imperfect Information. In Proceedings of the 36th International Colloquium on Automata, Languages and Programming (ICALP'09) (Lecture Notes in Computer Science), Vol. 5556. Springer, 200-211.

Vincent Gripon and Olivier Serre. 2011. Qualitative Concurrent Stochastic Games with Imperfect Information. CoRR abs/0902.2108 (2011).

F. Horn. 2008. Random Games. Ph.D. Dissertation. Université Denis-Diderot.

J.-F. Mertens and A. Neyman. 1982. Stochastic games have a value. In Proc. of the National Academy of Sciences USA, Vol. 79. 2145-2146.

A. Paz. 1971. Introduction to probabilistic automata. Academic Press.

M. O. Rabin. 1963. Probabilistic automata. Information and Control 6, 3 (1963), 230-245.

J. H. Reif. 1979. Universal games of incomplete information. In Proc. of STOC'79. ACM, 288-308.

Jérôme Renault. 2007. The value of repeated games with an informed controller. Technical Report. CEREMADE, Paris.

Jérôme Renault and Sylvain Sorin. 2008. Personal Communications. (2008).

Dinah Rosenberg, Eilon Solan, and Nicolas Vieille. 2003. Stochastic Games with Imperfect Monitoring. Technical Report 1376. Northwestern University.

L. S. Shapley. 1953. Stochastic games. In Proc. of the National Academy of Sciences USA, Vol. 39. 10951100.

Sylvain Sorin. 2002. A first course on zero-sum repeated games. Springer.

M. Tracol. 2011. Recurrence and transience for finite probabilistic tables. Theor. Comput. Sci 412, 12-14 (2011), 1154-1168.

J. von Neumann and O. Morgenstern. 1944. Theory of games and economic behavior. Princeton University Press.

Received ; revised ; accepted 